\title{
Tissue Optics and Photonics: Light-Tissue Interaction
}

\author{
Valery V. Tuchin ${ }^{1,2,3}$ \\ ${ }^{1}$ Saratov State University, 83 Astrakhanskaya str., Saratov 410012, Russia \\ ${ }^{2}$ Institute of Precision Mechanics and Control RAS, 24 Rabochaya str., Saratov 410028, Russia \\ ${ }^{3}$ Samara State Aerospace University (SSAU), 34 Moskovskoye Shosse, Samara 443086, Russia \\ e-mail: tuchinvv@mail.ru
}

\begin{abstract}
This is the second section of the review-tutorial paper describing fundamentals of tissue optics and photonics. As the first section of the paper was mostly devoted to description of biological tissue structures and their specificity related to interactions with light [1], this section 3 describes light-tissue interactions themselves that are caused by tissue dispersion, scattering, and absorption properties, including light reflection and refraction, absorption, elastic, and quasi-elastic scattering. The major tissue absorbers and modes of elastic scattering, including Rayleigh and Mie scattering, will be presented. (C) 2015 Samara State Aerospace University (SSAU).
\end{abstract}

Keywords: tissue optics; multiple scattering; quasi-elastic scattering; Doppler effect; absorption; index of refraction; random phase screen; speckles; optical coherence tomography (OCT); diffusion wave spectroscopy; polarized light; optical clearing

Paper \#2469 received 2015.05.30; accepted for publication 2015.06.29; published online 2015.06.30.

\section{References}

1. V. V. Tuchin, "Tissue Optics and Photonics: Biological Tissue Structures," J. of Biomedical Photonics \& Eng. 1(1), 3-21 (2015).

2. L. V. Wang. and H.-I. Wu, Biomedical Optics: Principles and Imaging, Wiley-Intersience, Hoboken, NJ (2007).

3. R. Splinter, and B. A. Hooper, An Introduction to Biomedical Optics, CRC Press, Taylor \& Francis Group, NY, London (2007).

4. T. Vo-Dinh (ed.), Biomedical Photonics Handbook, 2nd ed., CRC Press, Boca Raton (2014).

5. V. V. Tuchin, Tissue Optics: Light Scattering Methods and Instruments for Medical Diagnosis, 3rd ed., SPIE Press, Bellingham, WA (2015).

6. M. Born, and E. Wolf, Principles of Optics, 7th ed., Cambridge Univ. Press, Cambridge (1999).

7. G. S. Landsberg, Optics, 6th ed., Fizmatlit, Moscow (2006) [in Russian].

8. A. Akhmanov, S. Y. Nikitin, Physical Optics, Oxford University Press, Oxford (1997).

9. V. V. Tuchin, Lasers and Fiber Optics in Biomedical Science, 2nd ed., Fizmatlit, Moscow (2010) [in Russian].

10. V. V. Tuchin, "Light scattering study of tissues," Physics - Uspekhi 40(5), 495-515 (1997).

11. V. V. Tuchin (ed.), Handbook of Photonics for Biomedical Science, CRC Press, Taylor \& Francis Group, London (2010).

12. A. Wax, and V. Backman (eds.), Biomedical Applications of Light Scattering, McGraw-Hill, NY (2010).

13. D. A. Boas, C. Pitris, and N. Ramanujam (eds.), Handbook of Biomedical Optics, CRC Press, Taylor \& Francis Group, London (2011).

14. V. V. Tuchin, Dictionary of Biomedical Optics and Biophotonics, SPIE Press, Bellingham, WA (2012).

15. J. Popp, V. Tuchin, A. Chiou, and S. H. Heinemann (eds.), Handbook of Biophotonics: Basics and Techniques, vol.1, Wiley-VCH Verlag GmbH \& Co. KGaA, Weinheim (2011).

16. J. Popp, V. V. Tuchin, A. Chiou, and S. H. Heinemann (eds.), Handbook of Biophotonics: Photonics for Health Care, vol. 2, WILEY-VCH Verlag GmbH \& Co. KGaA, Weinheim (2011).

17. A. N. Bashkatov, E. A. Genina, and V. V. Tuchin, "Optical properties of skin, subcutaneous, and muscle tissues: a review," J. Innov. Opt. Health Sci. 4(1), 9-38 (2011).

18. S. L. Jacques, "Optical properties of biological tissues: A review," Phys. Med. Biol. 58(14), R37-R61 (2013).

19. R. K. Wang, and V. V. Tuchin, Advanced Biophotonics: Tissue Optical Sectioning, CRC Press, Taylor \& Francis Group, London (2013). 
20. S. L. Jacques, "Probing the nano-, micro-, and meso-scale structures of biological tissues using light scattering," International Conference on Laser Applications in Life Sciences (LALS), June 29 - July 2, Ulm, Germany (2014).

21. S. L. Jacques, "Quick analysis of optical spectra to quantify epidermal melanin and papillary dermal blood content of skin," J. Biophotonics 8(4), 309-316 (2015).

22. C. F. Bohren, and D.R. Huffman, Absorption and Scattering of Light by Small Particles, Wiley, NY (1983).

23. M. I. Mishchenko, L.D. Travis, and A.A. Lacis, Scattering, Absorption, and Emission of Light by Small Particles, Cambridge Univ. Press, Cambridge (2002).

24. R. G. Johnston, S. B. Singham, and G. C. Salzman, "Polarized light scattering" in Comments Mol. Cell. Biophys. 5(3), Gordon and Breach Sci. Publ. Inc., 171-192 (1988).

25. K. Frank, and M. Kessler (eds.), Quantitative Spectroscopy in Tissue, pmi Verlag, Frankfurt am Main (1992).

26. H. C. van de Hulst, Light Scattering by Small Particles, Wiley, New York (1957); reprint, Dover, NY (1981); Multiple Light Scattering. Tables, Formulas and Applications, Academic Press, NY (1980).

27. G. C. Beck, N. Akgun, A. Rück, and R. Steiner, "Design and characterization of a tissue phantom system for optical diagnostics," Lasers Med. Sci. 13, 160-171 (1998).

28. V. V. Tuchin (ed.), Handbook of Optical Biomedical Diagnostics, SPIE Press, Bellingham, Washington (2002).

29. E. P. Zege, A. P. Ivanov, and I. L. Katsev, Image Transfer through a Scattering Medium, Springer-Verlag, NY (1991).

30. A. Ishimaru, Wave Propagation and Scattering in Random Media, IEEE Press, NY (1997).

31. M. I. Mishchenko, L. D. Travis, and A. A. Lacis, Multiple Scattering of Light by Particles: Radiative Transfer and Coherent Backscattering, Cambridge University Press, NY (2006).

32. A. A. Kokhanovsky (ed.), Light Scattering Reviews 4: Single Light Scattering and Radiative Transfer, Springer, Heidelberg (2009).

33. A. A. Kokhanovsky (ed.), Light Scattering Reviews 5: Single Light Scattering and Radiative Transfer, Springer, Heidelberg (2010).

34. R. Graaff, J. G. Aarnoudse, J. R. Zijp, P. M. A. Sloot, F. F. M. de Mul, J. Greve, and M. H. Koelink, "Reduced light scattering properties for mixtures of spherical particles: a simple approximation derived from Mie calculations," Appl. Opt. 31, 1370-1376 (1992).

35. G. Müller, B. Chance, R. Alfano, et al. (eds.), Medical Optical Tomography: Functional Imaging and Monitoring, SPIE Inst. Adv. Opt. Techn. IS11, SPIE Press, Bellingham, WA (1993).

36. M. S. Patterson, "Noninvasive measurement of tissue optical properties: current status and future prospects" in Comments Mol. Cell. Biophys. 8, Gordon and Breach Sci. Publ. Inc., 387-417 (1995).

37. B. Chance, M. Cope, E. Gratton, N. Ramanujam, and B. Tromberg, "Phase measurement of light absorption and scatter in human tissue," Rev. Sci. Instrum. 69(10), 3457-3481 (1998).

38. O. Minet, G. Müller, and J. Beuthan (eds.), Selected Papers on Optical Tomography, Fundamentals and Applications in Medicine, MS147, SPIE Press, Bellingham, WA (1998).

39. J. C. Dainty (ed.), Laser Speckle and Related Phenomena, 2nd ed., Springer-Verlag, NY (1984).

40. J. W. Goodman, Statistical Optics, Wiley-Interscience Publication, NY, et al. (1985).

41. J. W. Goodman, Introduction to Fourier Optics, McGraw-Hill, NY (1996).

42. J. W. Goodman, Speckle Phenomena in Optics: Theory and Applications, Roberts \& Co., Englewood, CO (2007).

43. P. Zakharov, and F. Scheffold, "Advances in dynamic light scattering techniques" in Light Scattering Reviews 4: Single Light Scattering and Radiative Transfer, A.A. Kokhanovsky (ed.), Springer, Heidelberg, 433-468 (2009).

44. A. P. Shepherd, and P.Å. Öberg (eds.), Laser Doppler Blood Flowmetry, Kluwer, Boston (1990).

45. B. J. Berne, and R. Pecora, Dynamic Light Scattering: with Applications to Chemistry, Biology, and Physics, Dover Publications, Inc., Mineola, NY (2000).

46. D. A. Boas and K. Dunn, "Laser speckle contrast imaging in biomedical optics," J. Biomed. Opt. 15(1), 011109 (2010).

47. V. V. Tuchin (ed.), Advanced Optical Cytometry: Methods and Disease Diagnoses, Wiley-VCH Verlag GmbH \& Co. KGaA, Weinheim (2011).

48. V. V. Tuchin (ed.), Coherent-Domain Optical Methods: Biomedical Diagnostics, Environmental Monitoring and Material Science, 2nd ed., 2 vols., Berlin, Heidelberg, N.Y., Springer-Verlag (2013).

49. M. J. Leahy (ed.), Microcirculation Imaging, Wiley-VCH Verlag GmbH \& Co. KGaA, Weinheim (2012).

50. S. M. Daly, and M. J. Leahy, "GGo with the flow:' A review of methods and advancements in blood flow imaging," J. Biophotonics, 6(3), 217-255 (2013).

51. N. Yokoi, Y. Shimatani, M. Kyoso, H. Funamizu, and Y. Aizu, "Imaging of blood flow and blood concentration change in a frame rate using laser speckle: Methods for image analysis," Opt. Laser Tech. 64, 352-362 (2014). 
52. H. M. Varma, C. P. Valdes, A. K. Kristoffersen, J. P. Culver, and T. Durduran, "Speckle contrast optical tomography: A new method for deep tissue three-dimensional tomography of blood flow," Biomed. Opt. Express 5(4), 1275-1289 (2014).

53. P. Farzam, and T. Durduran, "Multidistance diffuse correlation spectroscopy for simultaneous estimation of blood flow index and optical properties," J. Biomed. Opt. 20(5), 055001 (2015).

54. D. A. Zimnyakov, J. D. Briers, and V. V. Tuchin, "Speckle technologies for monitoring and imaging of tissuelike phantoms," in Handbook of Optical Biomedical Diagnostics, V. V. Tuchin (ed.), SPIE Press, Bellingham, Washington, 987-1036 (2002).

55. I. V. Fedosov and V. V. Tuchin, "Laser Doppler and speckle techniques for bioflow measurements," in Coherent-Domain Optical Methods: Biomedical Diagnostics, Environmental Monitoring and Material Science, 2nd ed., V. V. Tuchin (ed.), Springer-Verlag Berlin, Heidelberg, NY, 487-564 (2013).

56. A. V. Priezzhev, V. V. Tuchin, and L. P. Shubochkin, Laser Diagnostics in Biology and Medicine, Nauka, Moscow (1989) [in Russian].

57. G. Popescu, "Quantitative phase imaging of nanoscale cell structure and dynamics," in Methods in Nano Cell Biology, B. P. Jena (ed.), Methods in Cell Biology 90, Academic Press, NY, 87-115 (2008).

58. G. Popescu, Quantitative Phase Imaging of Cells and Tissues, McGraw-Hill, NY (2011).

59. V. P. Ryabukho, "Diffraction of interference fields on random phase objects," in Coherent-Domain Optical Methods: Biomedical Diagnostics, Environmental and Material Science, V. V. Tuchin (ed.), Kluwer Academic Publishers, Boston, 235 -318 (2004).

60. A. Serov, and T. Lasser, "High-speed laser Doppler perfusion imaging using an integrating CMOS image sensor," Opt. Express 13(17), 6416-6428 (2005).

61. I. V. Meglinski, and V. V. Tuchin, "Diffusing wave spectroscopy: Application for blood flow diagnostics," in Coherent-Domain Optical Methods: Biomedical Diagnostics, Environmental Monitoring and Material Science, 2nd ed., V. V. Tuchin (ed.), Springer-Verlag, Berlin, Heidelberg, NY, 149-167 (2013).

62. W. A. Shurkliff, Polarized Light. Production and Use, Harvard Univ., Cambridge, Mass (1962).

63. W. A. Shurkliff, and S. S. Ballard, Polarized Light, Van Nostrand, Princeton (1964).

64. D. S. Kliger, J. W. Lewis, and C. E. Randall, Polarized Light in Optics and Spectroscopy, Academic, Boston (1990).

65. E. Collet, Polarized Light. Fundamentals and Applications, Dekker, NY (1993).

66. R. M. A. Azzam, and N. M. Bashara, Ellipsometry and Polarized Light, Elsevier Science, Amsterdam (1994).

67. A. Z. Dolginov, Y. N. Gnedin, and N.A. Silant'ev, Propagation and Polarization of Radiation in Cosmic Media, Gordon and Breach, Basel (1995).

68. C. Brosseau, Fundamentals of Polarized Light: A Statistical Optics Approach, Wiley, NY (1998).

69. M. I. Mishchenko, J. W. Hovenier, and L. D. Travis (eds.), Light Scattering by Nonspherical Particles, Academic Press, San Diego (2000).

70. A. A. Kokhanovsky, Polarization Optics of Random Media. Springer-Verlag, Berlin, Heidelberg, NY (2003).

71. V. V. Tuchin, L. V. Wang, and D. A. Zimnyakov, Optical Polarization in Biomedical Applications, Springer, NY (2006).

72. L. V. Wang, G. L. Coté, and S. L. Jacques (eds.), "Special Section on Tissue Polarimetry," J. Biomed. Opt. 7(3), 278-397 (2002).

73. S. L. Jacques, J. C. Ramella-Roman, and K. Lee, "Imaging skin pathology with polarized light," J. Biomed. Opt. 7(3), 329-340 (2002).

74. J. Ramella-Roman, S. Prahl, and S. Jacques, "Three Monte Carlo programs of polarized light transport into scattering media: part I," Opt. Express 13(12), 4420-4438 (2005).

75. J. Ramella-Roman, S. Prahl, and S. Jacques, "Three Monte Carlo programs of polarized light transport into scattering media: part II," Opt. Express 13(25), 10392-10405 (2005).

76. N. Ghosh, and I. A. Vitkin, "Concepts, challenges and applications of polarized light in biomedicine: A tutorial review,” J. Biomed. Opt. 16(11), 110801 (2010).

77. B. Kunnen, C. Macdonald, A. Doronin, S. Jacques, M. Eccles, and I. Meglinski, “Application of circularly polarized light for non-invasive diagnosis of cancerous tissues and turbid tissue-like scattering media," J. Biophotonics 8(4), 317-323 (2015).

78. V. V. Tuchin, “Optical immersion as a new tool to control optical properties of tissues and blood," Laser Phys. 15(8), 1109-1136 (2005).

79. V. V. Tuchin, "Optical clearing of tissue and blood using immersion method," J. Phys. D: Appl. Phys. 38, 2497-2518 (2005).

80. V. V. Tuchin, Optical Clearing of Tissues and Blood, PM 154, SPIE Press, Bellingham, WA (2006).

81. V. V. Tuchin, “A clear vision for laser diagnostics,” IEEE J. Select. Tops. Quant. Electr. 13(6), 1621-1628 (2007).

82. V. V. Tuchin (ed.), Handbook of Optical Sensing of Glucose in Biological Fluids and Tissues, CRC Press, Taylor \& Francis Group, London (2009). 
83. E. A. Genina, A. N. Bashkatov, and V. V. Tuchin, "Tissue optical immersion clearing," Expert Rev. Med. Devices 7(6), 825-842 (2010).

84. E. A. Genina, A. N. Bashkatov, K. V. Larin, and V. V. Tuchin, "Light-tissue interaction at optical clearing" in Laser Imaging and Manipulation in Cell Biology, F.S. Pavone (ed.), Wiley-VCH Verlag GmbH \& Co. KGaA, Weinheim, 115-164 (2010).

85. K. V. Larin, M. G. Ghosn, A. N. Bashkatov, E. A. Genina, N. A. Trunina, and V. V. Tuchin, "Optical clearing for OCT image enhancement and in-depth monitoring of molecular diffusion,” IEEE J. Select. Tops. Quant. Electr. 18 (3) 1244-1259 (2012).

86. D. Zhu, K. V. Larin, Q. Luo, and V. V. Tuchin, "Recent progress in tissue optical clearing," Laser Photonics Rev. 7(5), 732-757 (2013).

87. D. Zhu, Q. Luo, and V. V. Tuchin, "Tissue Optical Clearing," in Advanced Biophotonics: Tissue Optical Sectioning, R. K. Wang, and V. V. Tuchin (eds.), CRC Press, Taylor \& Francis Group, Boca Raton, London, NY, 621-672 (2013).

88. R. K. Wang, and V.V. Tuchin, "Optical coherence tomography: light scattering and imaging enhancement," in Coherent-Domain Optical Methods: Biomedical Diagnostics, Environmental Monitoring and Material Science, 2nd ed., V. V. Tuchin (ed.), Springer-Verlag, Berlin, Heidelberg, NY, 665-742 (2013).

89. J. Wang, Y. Zhang, P. Li, Q. Luo, and D. Zhu, "Review: Tissue Optical Clearing Window for Blood Flow Monitoring (Invited Paper),” IEEE J. Select. Tops Quant. Electr. 20(2), 6801112 (2014).

90. O. Nadiarnykh, and P. J. Campagnola, "SHG and optical clearing," in Second Harmonic Generation Imaging, F.S. Pavone, and P.J. Campagnola (eds.), CRC Press, Taylor \& Francis Group, Boca Raton, London, NY, 169-189 (2014).

91. E. A. Genina, A. N. Bashkatov, Y. P. Sinichkin, I. Y. Yanina, and V. V. Tuchin, "Optical clearing of biological tissues: prospects of application in medical diagnostics and phototherapy," J. of Biomedical Photonics \& Eng. 1(1), 22-58 (2015).

92. X.-C. Zhang, and J. Xu, Introduction to THz Wave Photonics, Springer, NY (2010).

93. M. M. Nazarov, A. P. Shkurinov, V. V. Tuchin, and X.-C. Zhang, "Terahertz tissue spectroscopy and imaging" in Handbook of Photonics for Biomedical Science, V. V. Tuchin (ed.), CRC Press, Taylor \& Francis Group, London, 591-617 (2010).

\section{Contents}

1 Introduction

2 Biological tissue structures

2.1 General definitions and characteristics

2.2 Soft tissues

2.3 Hard tissues

2.4 Tissue structural anisotropy

2.5 Blood, lymph and other bioliquids

3 Light-tissue interaction: absorption, scattering and polarization

3.1 Light reflection and refraction

3.2 Light absorption and elastic scattering

3.2.1 Absorption and tissue absorbers

3.2.2 Scattering - basic definitions

3.2.3 Rayleigh scattering

3.2.4 Mie scattering

3.2.5 Multiple scattering

3.3 Scattering of the coherent light

3.3.1 Random phase screen concept and speckle formation

3.3.2 Speckle interferometry

3.3.3 Low-coherent light interferometry - optical coherence tomography

3.4 Dynamic light scattering

3.4.1 Quasi-elastic light scattering (QELS) and

Doppler effect

3.4.2 Dynamic speckles

3.4.3 Speckle pattern blurring effect - full-field

velocity measurements
3.5 Diffusion wave spectroscopy

3.6 Interaction of the polarized light with tissues

3.6.1 Definitions

3.6.2 Single scattering and quasi-ordered tissues

3.6.3 Multiple scattering

3.7 Refractive index and controlling of light interaction with tissues

\section{Light-tissue interaction: absorption, scattering and polarization}

\subsection{Light reflection and refraction}

A biological tissue is a dielectric medium whose average refractive index is higher than that of air [1-5]. Thus at interaction with tissue surface the light radiation is able to undertake partial reflection at the tissue/air interface (Fresnel reflection), while the remaining part penetrates the tissue (see Fig. 3.1a) [6-8]. Light refraction is the change in direction of a ray of light when passing obliquely from one medium into another in which the light speed (refractive index) is different. Light refraction is characterized by the relative index of refraction, $m$, of these two media with $n_{1}$ and $n_{2}$ indices, $n_{2}>n_{1}$. For different human tissues and tissue components, refractive index (RI) in the visible/NIR wavelength range varies from a value a little bit higher than for water due to influence of some organic 


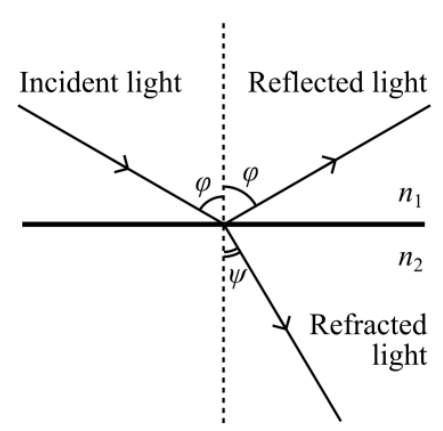

a

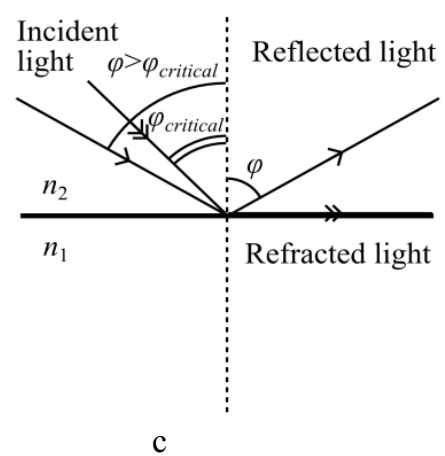

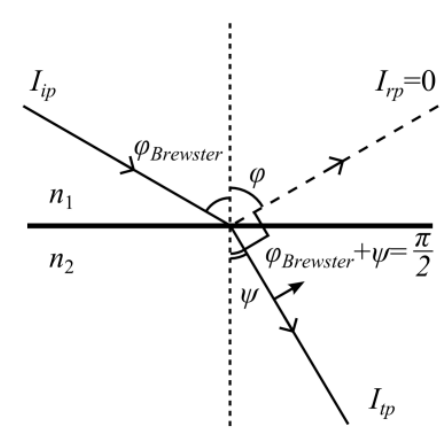

b

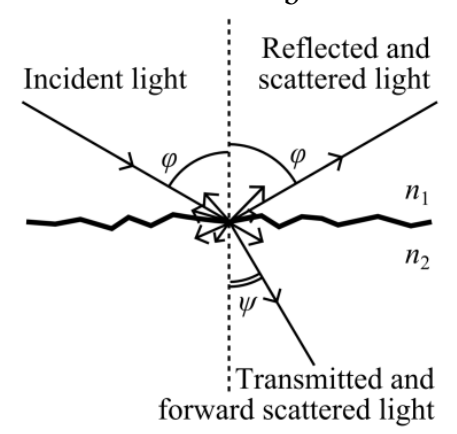

d

Fig. 3.1 Light interaction with a nonscattering tissue with mean index of refraction $n_{2} \equiv n_{\text {tissue }}, n_{1}$ is the index of refraction of a surrounding medium; $n_{\text {tissue }}>n_{1}$ : (a) illustrates light reflection by and transmission through the flat interface of a tissue surface and surrounding medium; (b) illustrates the Brewster angle $\varphi_{\text {Brewster }}$ - the angle of incidence at which light polarized in the incident light plane (p) is perfectly transmitted; for skin stratum corneum with $n_{2} \cong 1.5$ and $n_{1}=1$, $\varphi_{\text {Brewster }} \cong 57^{\circ}$; (c) illustrates total internal reflection of light when it goes from a medium with a higher index of refraction $\left(n_{2}\right)$ to a medium with the lower one $\left(n_{1}\right)$ in the case of a flat interface between two nonscattering media; for skin stratum corneum with $n_{2} \cong 1.5$ and $n_{1}=1, \varphi_{\text {critical }} \cong 45^{\circ}$; (d) illustrates light reflection, transmission and scattering by rough interface of a scattering tissue and surrounding media.

components, $n_{2} \cong 1.35$ for interstitial fluid, to 1.62 for tooth enamel.

In Fig. 3.1b, p polarization denotes that the electric field is polarized parallel to the plane of incidence (the same plane as the incident, reflected and transmitted rays). As well as s polarization denotes that the electric field is polarized perpendicular the plane of incidence. To describe polarized light reflectance $\left(R_{p}\right.$ and $\left.R_{s}\right)$ by and transmittance $\left(T_{p}\right.$ and $\left.T_{s}\right)$ through a flat (not rough) interface of transparent (not scattering) tissue Fresnel formulas can be used [6-8]

$$
\begin{aligned}
& R_{p}=\frac{\tan ^{2}(\varphi-\psi)}{\tan ^{2}(\varphi+\psi)} \\
& T_{p}=\frac{\sin 2 \varphi \sin 2 \psi}{\sin ^{2}(\varphi+\psi) \cos ^{2}(\varphi-\psi)} \\
& R_{s}=\frac{\sin ^{2}(\varphi-\psi)}{\sin ^{2}(\varphi+\psi)} \\
& T_{s}=\frac{\sin ^{2} \varphi \sin 2 \psi}{\sin ^{2}(\varphi+\psi)}
\end{aligned}
$$

where $R_{p}, R_{s}$ and $T_{p}, T_{s}$ are reflectance and transmittance, respectively, or the ratios of the reflected $\left(I_{r p}, I_{r s}\right)$ and transmitted $\left(I_{t p}, I_{t s}\right)$ intensities to the incident intensity for each polarization state in the incident light plane $(p)$ and the orthogonal plane ( $s), I_{i p}$ and $I_{i s}$, respectively; $\varphi$ is the incident angle (equal to reflection angle) and $\psi$ is the angle of refraction; these angles are defined by the indices in accordance with the Snell's law

$$
n_{1} \sin \varphi=n_{2} \sin \psi
$$

and can be expressed in terms of the relative index of refraction $m$

$$
\frac{n_{2}}{n_{1}}=m
$$

For the specific light incident angles, the reflectivity and transmittance are described by the following formulas:

At the normal incidence to the surface $\left(\varphi=0^{\circ}\right)$,

$$
R_{p}=R_{s}=R=\left(\frac{m-1}{m+1}\right)^{2}
$$




$$
T_{p}=T_{s}=T=\frac{4 m}{(m+1)^{2}}
$$

At the incidence angle that produces a rectangular angle between the reflected and refracted rays which is called the Brewster angle $\left(\varphi_{\text {Brewster }}+\psi=90^{\circ}\right)$, or

$$
\varphi_{\text {Brewster }}=\arctan m
$$

$R_{p}=0, R_{s} \neq 0$, i.e., this is an angle of incidence at which light with a particular polarization $p$ is perfectly transmitted, at other angles the reflected light is partially polarized (see Fig. 3.1b);

At the incidence of light from the medium with a higher RI $\left(n_{2}\right)$ into the medium with the lower RI $\left(n_{1}\right)$ (see Fig. 3.1c) a so called critical angle $\varphi_{\text {critical }}$ exists for which if incident angle $\varphi \geq \varphi_{\text {critical }}$ total internal reflection (no transmitted light) is created

$$
\varphi_{\text {critical }}=\arcsin m \text {. }
$$

All these formulas are applicable only to homogeneous media with flat interface between. Thus they could be applied only locally for a limited number of tissues such as healthy eye cornea, lens and vitreous body (see Fig. 2.6) [1]. However, the major types of tissues are optically inhomogeneous (turbid) and absorptive multilayered media with rough interfaces. In that case light scattering from the interface and from bulk of a tissue will influence light reflection and transmission with the redistribution of light in the space (see Fig. 3.1d).

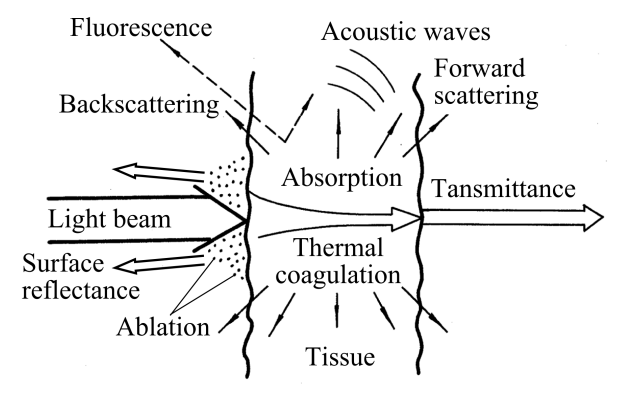

Fig. 3.2 Schematic representation of different modes of light interaction with tissues [9].

Actually, in tissues multiple scattering and absorption processes are responsible for light beam broadening and eventual decay as it travels through a tissue, whereas bulk scattering is a major cause of the dispersion of a large fraction of radiation in the backward direction (see Figs. 3.2 and 3.3) [9]. Therefore light propagation within a tissue depends on the scattering and absorption properties of its components: cells, cell organelles, and various fiber structures. The size, shape, and density of these structures, their refractive index, and the polarization state of the incident light predetermine the character of light propagation in tissues.

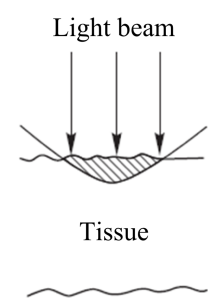

a

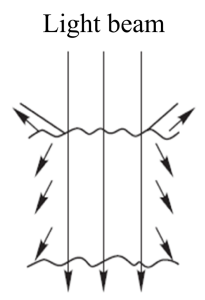

b

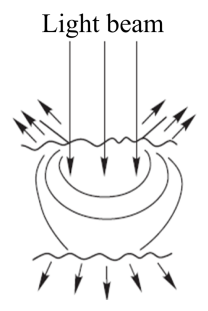

$\mathrm{c}$
Fig. 3.3 Light beam absorption and scattering by a tissue: a - absorption prevails, is typical for UV or MIR/FIR radiation, no light propagates into tissue; $b$ absorption and scattering are approximately equal to each other, is typical for some range in the NIR, light beam propagates well with some amount of scattered radiation; c - scattering prevails, is typical for visible and NIR, a significant light beam broadening with diffusive irradiation of large tissue volume [9].

\subsection{Light absorption and elastic scattering}

\subsubsection{Absorption and tissue absorbers}

The process of light absorption is the transformation of light energy to some other form of energy, i.e. heat, sound, fluorescence as the light transverses tissue. Commonly an absorbing medium consists of absorption centers that are particles or molecules that absorb light. To characterize absorption of a medium an absorption coefficient $\mu_{a}$ is introduced as the reciprocal of the distance $d$ over which light of intensity $I_{0}$ is attenuated to $I(d)=\frac{I_{0}}{e} \approx 0.37 I_{0}$, which follows from the exponential law for light propagation in a tissue layer of thickness $d$ (Fig.3.4) [2-5]

$$
I(d)=I_{0} \exp \left(-\mu_{a} d\right)
$$

where $I(d)$ is the intensity of transmitted light, W/cm ${ }^{2}$; $\mu_{a}$ is typically expressed in $\mathrm{cm}^{-1}$.

Behind this definition is a fundamental process of photon absorption that is characterized by an effective cross section, i.e., the ability of a molecule to absorb a photon of a particular wavelength. Although the units are given as an area, it does not refer to an actual size area, at least partially because the density or state of the target molecule will affect the probability of absorption. Quantitatively, the number $\mathrm{d} N$ of photons absorbed, between the points $x$ and $x+\mathrm{d} x$ along the path of a light beam is the product of the number $N$ of photons penetrating to depth $x$ multiplied by the absorption cross section $\sigma_{a b s}\left(\mathrm{~cm}^{2}\right)$ and by the number of absorbing molecules per unit volume $\rho_{M}\left(\mathrm{~cm}^{-3}\right)$ (Fig.3.4):

$$
\frac{\mathrm{d} N}{\mathrm{~d} x}=-\rho_{M} \sigma_{a b s} N
$$


where

$$
\mu_{a}=\rho_{M} \sigma_{a b s} .
$$

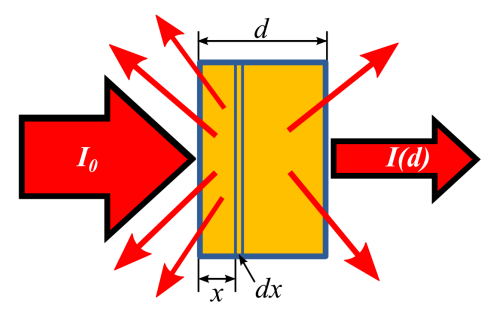

Fig. 3.4 Light beam attenuation due to absorption and scattering.

In spectroscopy a few terms are commonly used, such as absorbance that is the ratio of the absorbed light intensity to the incident intensity, thus, it is a dimensionless quantity, and absorption spectrum that is the spectrum formed by light that has passed through a medium in which light of certain wavelengths was absorbed.

The wavelength dependent optical depth $\tau_{\lambda}$ which is defined as,

$$
\tau_{\lambda}=\ln \left(\frac{I_{0}}{I}\right)=\mu_{a} d=\varepsilon_{\lambda} c_{a} d
$$

is also often used. Here $\varepsilon_{\lambda}$ is the extinction coefficient at the wavelength $\lambda$ in $\mathrm{L} /(\mathrm{mmol} \cdot \mathrm{cm}), \quad c_{a}$ is the concentration of absorbing substance in $\mathrm{mmol} / \mathrm{L}$, and $d$ is in $\mathrm{cm}$.

Absorption spectra can be also expressed in terms of the wavelength dependence of absorption coefficient. Such UV-visible-IR spectra for the major tissue absorbers (chromophores): water, lipids, oxy- and deoxyhemoglobin are presented in Fig. 3.5. The diagnostic/therapeutic spectral window, where tissue absorption is minimal and light penetrates deeper into a tissue, is found between $700 \mathrm{~nm}$ and $1100 \mathrm{~nm}$, and two more narrow and less transparent windows are around $1300 \mathrm{~nm}$ and $1600 \mathrm{~nm}$. Because water is the major component of any tissue, the absorption coefficient of water is often dominates in the total absorption coefficient of a tissue in a particular wavelength range, especially in the IR (see Fig. 3.5).

Absorption is only one way by which light can interact with the tissue to induce photothermal and photochemical effects followed up by a chain of biological effects $[2-5,10-21]$. Absorption of the UV and visible/NIR light in tissue is due to electronic excitation of aromatic or conjugated unsaturated chromophores. A chromophore is a chemical that absorbs light with a characteristic spectral pattern. There are many kinds of chromophores in the tissue, but a few major chromophores predominantly determine the optical absorption within each tissue layer. As an example, spectral ranges of absorption of the main human skin chromophores are presented in Fig.3.6. Proteins found in the epidermis contain the aromatic amino acids tryptophan and tyrosine which have a characteristic absorption band near 270-280 nm; urocanic acid and the nucleic acids also contribute to this absorption band with a maximum near 260-270 nm. Epidermal melanin plays an important role in limiting the penetration depth of light in the skin: it effectively absorbs at the wavelengths from 300 to $1000 \mathrm{~nm}$, but the strongest absorption occurs at shorter wavelengths, in the near UV spectral range.
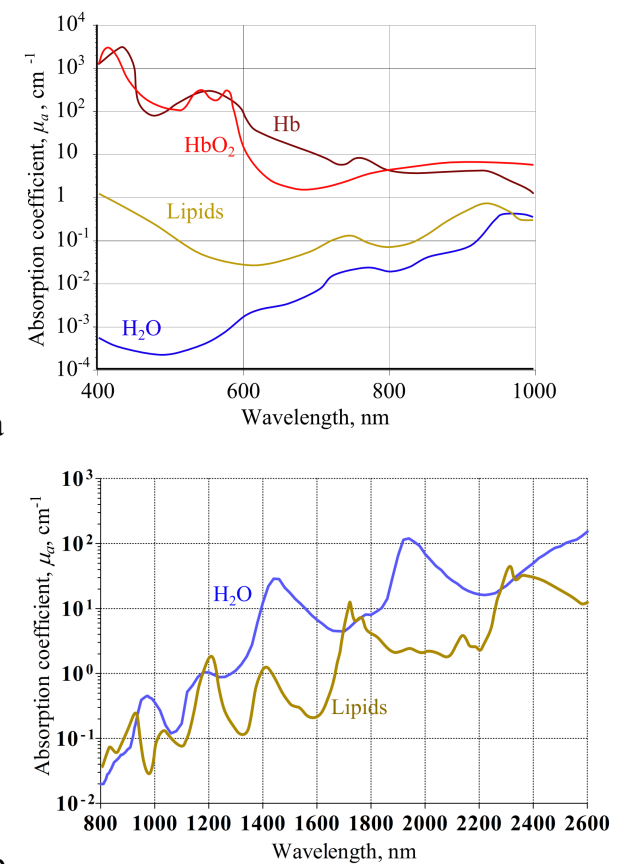

b

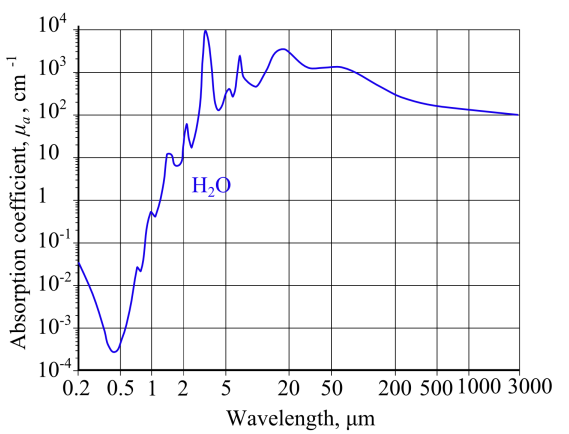

Fig. 3.5 Absorption spectra (expressed in terms of absorption coefficient, $\left.\mu_{a}, \mathrm{~cm}^{-1}\right)$ of the main tissue chromophores: Visible-NIR range (water $-\mathrm{H}_{2} \mathrm{O}$, Lipids, oxyhemoglobin $-\mathrm{HbO}_{2}$, deoxyhemoglobin $-\mathrm{Hb}$ ) (a); NIR (water $-\mathrm{H}_{2} \mathrm{O}$, Lipids - in the form of human fat) (b); UV-visible-IR (water) (c) [2-5].

The same as for any soft tissue in the IR spectral range, the skin absorption is essentially determined by the absorption of water contained in the skin (see Fig. 3.5). The oxyhemoglobin, deoxyhemoglobin, bilirubin, carotenoids and porphyrins are the major chromophores of skin derma (see Fig. 3.6) $[2-5,10-21]$. The oxyhemoglobin has its strongest absorption band at 415 $\mathrm{nm}$ (Soret band), and it has two secondary absorption 
bands at 542 and $577 \mathrm{~nm}$ (Q-bands). The deoxyhemoglobin has a primary absorption band at 430 $\mathrm{nm}$ and it has a single secondary absorption band at 555 $\mathrm{nm}$. Both hemoglobins exhibit the lowest absorption at wavelengths longer than $620 \mathrm{~nm}$. The bilirubin has two relatively broad absorption bands near 330 and $460 \mathrm{~nm}$.

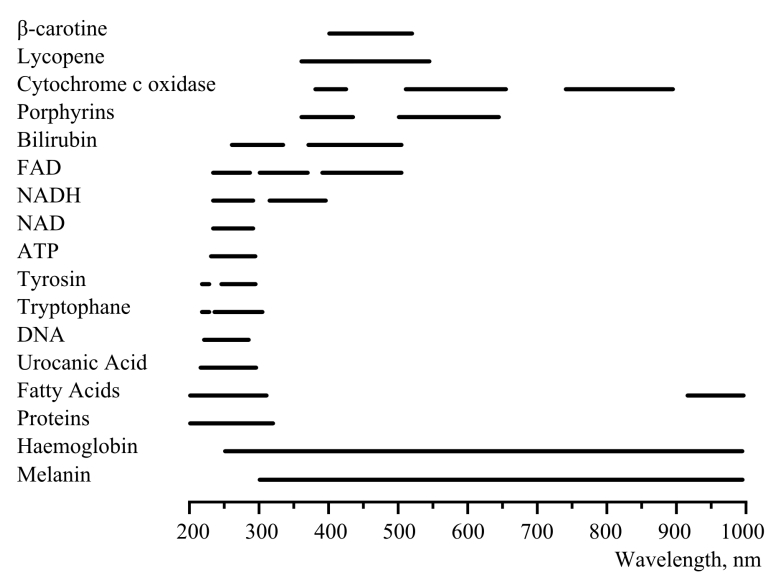

Fig. 3.6 Spectral ranges of absorption of the main human skin and other tissue chromophores; FAD flavin dinucleotide, NADH - the reduced form of coenzyme nicotinamide adenine dinucleotide (NAD), ATP - adenosine triphosphate.

Porphyrin-based fundamental biological representatives in tissues include not only hemoglobin but also other heme (iron contained) proteins, such as myoglobin and cytochromes, and vitamin B-12 (cobalt contained), and several others. Heme proteins serve many roles, like $\mathrm{O}_{2}$ storage and transport (haemoglobin and myoglobin), electron transport (cytochromes $b$ and $c$ ), and $\mathrm{O}_{2}$ activation and utilization (cytochrome $\mathrm{c}$ oxidase). As shown in Fig. 3.5a, the UV/visible absorption spectrum of the highly conjugated porphyrin macrocycle exhibits an intense feature (absorption coefficient $\sim 3 \times 10^{3} \mathrm{~cm}^{-1}$ ) at about $400 \mathrm{~nm}$ (the "Soret" band), followed by several weaker absorptions (Qbands) at higher wavelengths (from 450 to $700 \mathrm{~nm}$ for different heme proteins).

The enzyme cytochrome c oxidase (or cytochrome complex IV, or cytochrome- $a a_{3}$ ) is a large transmembrane protein complex located within the cell mitochondria. It catalyzes the final step of the respiratory chain in which oxygen is reduced to water. The enzyme contains two copper centers, denoted $\mathrm{CuA}$ and $\mathrm{CuB}$. When the enzyme is oxidized, the $\mathrm{CuA}$ center is responsible for the distinctive broad absorption band around $820-830 \mathrm{~nm}$. This band disappears when the enzyme is reduced, which allows one by measuring the amplitude of the band to track oxygen utilization directly to the site of ATP synthesis.

Myoglobin is found in muscle and its high affinity for oxygen means that it does not offload its oxygen until very low tissue oxygen pressures are reached. In muscle it serves as a reservoir to store and then slowly release the oxygen delivered by blood hemoglobin. The NIR absorption spectrum of myoglobin is essentially identical to that of hemoglobin, so that it is not possible to monitor it independently without resorting to a supplementary technique.

\subsubsection{Scattering - basic definitions}

Tissues are not only absorbing but also inhomogeneous media with different levels of organization that include cells, cell organelles and inclusions (see Figs. 2.2 [1] and 3.7), and different fiber and tubular/lamellar structures (see Figs. 2.5-2.11) [1]. In view of the great diversity and structural complexity of tissues, the development of an adequate optical model accounting for the scattering and absorption of light is often the most complex step of a study [2-5, 10-21]. Many tissues are composed of structures with a wide range of sizes (see Fig. 3.7), and can be represented as a system of discrete scattering particles. Such model has been used to describe the angular dependence of the intensity and polarization properties of scattered radiation. Blood is one of the biological examples of a disperse system that entirely corresponds to the model of discrete particles.

Biological media are often modeled as ensembles of homogeneous spherical particles with refractive index higher than surroundings (see Fig. 3.8), since many cells, cell organelles and biological macromolecules are close in shape to spheres or ellipsoids [2-5, 10-21]. A system of noninteracting spherical particles is the simplest tissue model. The Rayleigh and Mie theories or their combination are basic to calculate tissue scattering properties. In particular, Mie theory rigorously describes the diffraction (elastic scattering) of light by a spherical particle. The advances of this theory account the structures of the spherical particles, namely, the multilayered spheres and the spheres with radial nonhomogeneity, anisotropy, and optical activity.

For connective tissue which composed of fiber structures, a system of long cylinders is the most appropriate model to describe light scattering. Muscular tissue, skin dermis, dura mater, eye cornea and sclera belong to this type of tissue formed essentially by collagen fibrils. The solution of the problem of light scattering by a single homogeneous or multilayered cylinder is also well understood.

At transport (travel) in the inhomogeneous (turbid) medium with absorption a photon (light wave) changes its direction (wave vector) due to reflection, refraction, and diffraction on microscopic internal structures, and can be absorbed by an appropriate molecule on its way [2-5, 10-21]. Such structures which are smaller or comparable with the wavelength of the propagating light are called commonly scatterers and light scattering means change in direction of light propagation in a turbid medium. There are a number of parameters that describe scattering process. Scattering angle is related to photon scattered by a particle so that its trajectory is deflected by a deflection (scattering) angle $\theta$ in the scattering plane and by an azimuthal angle of scattering $\varphi(0$ to $2 \pi)$ in the plane perpendicular to the scattered photon trajectory (Fig. 3.9). Scattering plane is a plane 


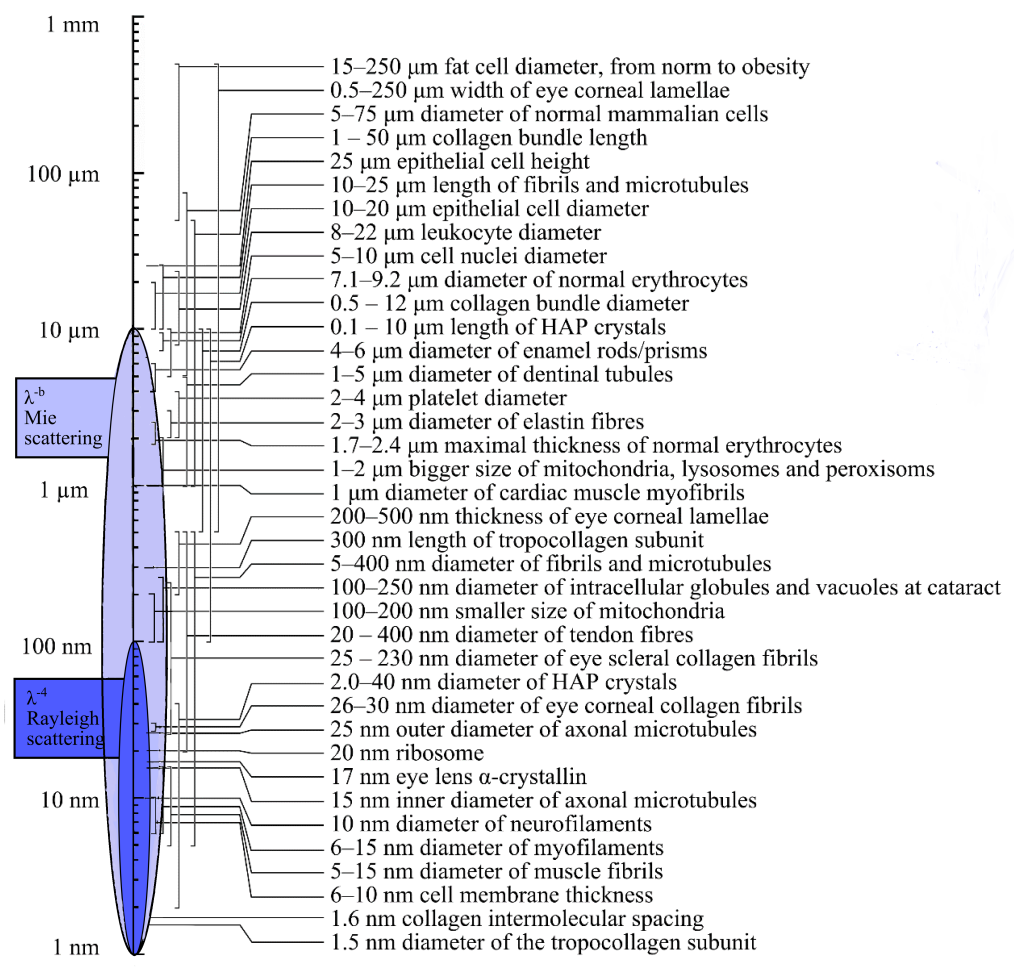

Fig. 3.7 Light scattering tissue and cell structures

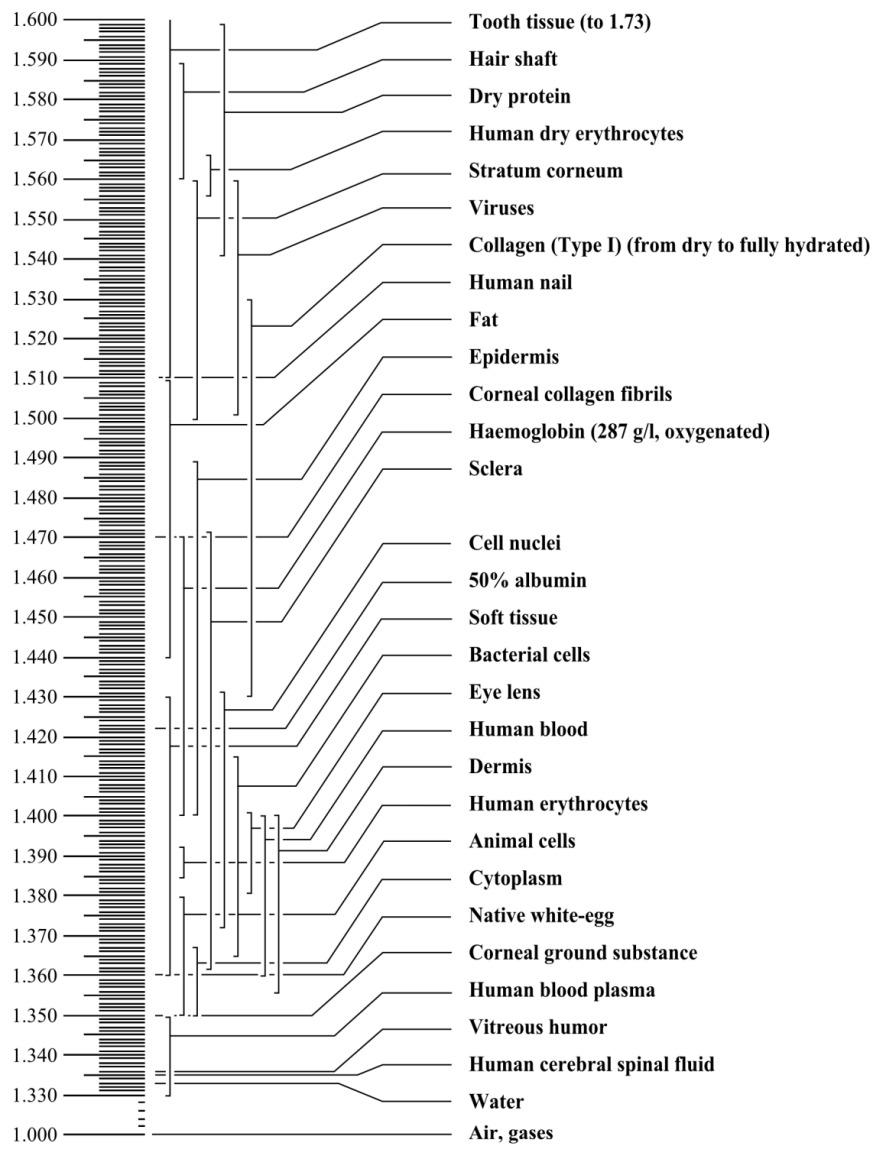

Fig. 3.8 Index of refraction of tissue and cell structures, the RI values depend on the wavelength which is sometimes in the range from UV to NIR, but mostly in the visible. 
defined by positions of a light source, a scattering particle, and a detector.

To characterize scattering efficiency of a medium a scattering coefficient $\mu_{s}$ is introduced, in a nonabsorbing sample it is defined as the reciprocal of the distance $d$ over which light of intensity $I(d=0)=I_{0}$ is attenuated (due to scattering) to $I(d)=\frac{I_{0}}{e} \approx 0.37 I_{0}$, which follows from the exponential law for light propagation in a tissue layer of thickness $d$ (Fig.3.4)

$$
I(d)=I_{0} \exp \left(-\mu_{s} d\right)
$$

where $I(d)$ is the intensity of transmitted light, $\mathrm{W} / \mathrm{cm}^{2}$; $\mu_{s}$ is typically expressed in $\mathrm{cm}^{-1}$.

Behind this definition is also a fundamental process of photon scattering that is characterized by a photon scattering cross section, i.e., the ability of a particle to scatter a photon of a particular wavelength and polarization. Although the units are given as an area, it does not refer to an actual size area. Quantitatively, the number $\mathrm{d} N$ of photons scattered, between the points $x$ and $x+\mathrm{d} x$ along the path of a light beam is the product of the number $N$ of photons penetrating to depth $x$ multiplied by the scattering cross section $\sigma_{s c a}\left(\mathrm{~cm}^{2}\right)$ and by the number of scattering particles per unit volume $\rho_{p}$ $\left(\mathrm{cm}^{-3}\right)$ (Fig.3.4):

$$
\frac{\mathrm{d} N}{\mathrm{~d} x}=-\rho_{p} \sigma_{s c a} N
$$

where

$$
\mu_{s}=\rho_{p} \sigma_{s c a} .
$$

Equation (3.15) is valid if scattering is not strong and only the unscattered portion of transmitted light beam (so called ballistic photons) are detected. Such regime could be more or less realized for thin tissue layers when absorption is high enough to eliminate multiple scattering events. Therefore, a collimated (laser) beam is attenuated in a thin tissue layer of thickness $d$ in accordance with the Bouguer-Beer-Lambert law

$$
I(d)=\left(1-R_{F}\right) I_{0} \exp \left(-\mu_{t} d\right)
$$

where $I(d)$ is the intensity of transmitted light measured using a distant photodetector with a small aperture (online or collimated transmittance), W/ $\mathrm{cm}^{2} ; R_{F}$ is the coefficient of Fresnel reflection; at the normal beam incidence, $R_{F}=\frac{(m-1)^{2}}{(m+1)^{2}} ; m$ is the relative mean refractive index of tissue and surrounding medium of the incident half-space (see Eq. (3.7)); $I_{0}$ is the incident light intensity, $\mathrm{W} / \mathrm{cm}^{2}$;

$$
\mu_{t}=\mu_{a}+\mu_{s}
$$

is the interaction or total attenuation coefficient. The attenuation is a decrease in energy per unit area of a light beam which occurs as the distance from the source increases and is caused by absorption and scattering.

Often such characteristic of turbid materials and tissues as albedo for single scattering is useful for prediction of light propagation in a tissue. Albedo is the ratio of the scattering to extinction coefficient,

$$
\Lambda=\frac{\mu_{s}}{\mu_{t}} .
$$

It ranges from zero for a completely absorbing medium to unity for a completely scattering medium.

Besides scattering coefficient and albedo, scattering process is also characterized by a so called scattering phase function - the function that describes the scattering properties of the medium and is in fact the probability density function for a photon travelling in some direction to be scattered in some new direction $p(\theta, \varphi)$. Figure 3.9 illustrates geometry of the scattering of light by a particle, where the incident light beam $\left(\vec{s}_{0}\right)$ is parallel to the $z$-axis and $\theta$ and $\varphi$ are the scattering angles in the scattering plane and in the plane perpendicular to the scattering plane, respectively [22]. Scattering phase function characterizes an elementary scattering act. If scattering is symmetric relative to the direction of the incident wave, then the phase function depends only on the scattering angle $\theta$ (angle between two directions, new $\left(\vec{s}_{1}\right)$ and former one $\left.\left(\vec{s}_{0}\right)\right), p(\theta)$.

Scattering anisotropy factor, $g$, is a major parameter of $p(\theta)$ and is a measure of the amount of forward direction retained after a single scattering event [2-5, $10-23$ ]. If a photon is scattered by a particle so that its trajectory is deflected by a scattering angle $\theta$, then the component of the new trajectory which is aligned in the forward direction is presented as $\cos \theta$. There is an average scattering angle and the mean value of $\cos \theta$ is defined as the anisotropy factor

$$
g \equiv\langle\cos \theta\rangle=\int_{0}^{\pi} p(\theta) \cos \theta \cdot 2 \pi \sin \theta \mathrm{d} \theta
$$

The value of $g$ varies in the range from -1 to $1: g=0$ corresponds to isotropic (Rayleigh) scattering, $g=1$ to total forward scattering (Mie scattering by large particles), and -1 to total backward scattering. The assumption of random distribution of the scattering particles in a medium (i.e. the absence of a spatial correlation of tissue structures) leads to normalization 


$$
\int_{0}^{\pi} p(\theta) 2 \pi \sin \theta \mathrm{d} \theta=1
$$

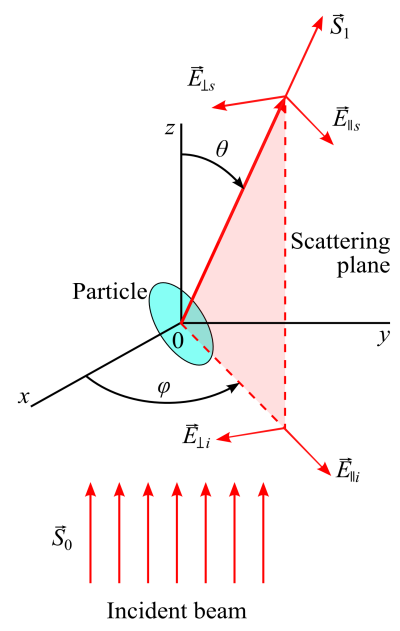

Fig. 3.9 Geometry of the scattering of light by a particle located at the origin 0 . The polarized incident light beam $\left(\vec{s}_{0}\right)$ is parallel to the $z$-axis [22]. Two orthogonal linear polarization components of the incident light field are presented as vectors $\vec{E}_{\| i}$ and $\vec{E}_{\perp i}$ in parallel and perpendicular to the scattering plane, respectively. $\theta$ and $\varphi$ are the scattering angles in the scattering plane and in the plane perpendicular to the scattering plane, respectively. A detector is located at distance $r$ from the origin along the vector $\overline{s_{1}}$, where two orthogonal polarization components $\vec{E}_{\| s}$ and $\vec{E}_{\perp s}$ of scattered light are coming.

\subsubsection{Rayleigh scattering}

If a particle is small with respect to the wavelength of the incident light, its scattering can be described as if it is a single dipole, the so called Rayleigh theory is applicable under the condition that $m(2 \pi a / \lambda)<<1$, where $m$ is the relative refractive index of the scatterers, $(2 \pi a / \lambda)$ is the size parameter, $a$ is the radius of the particle, and $\lambda$ is the wavelength of the incident light in a medium [6-8, 22, 23]. For this theory, the scattered irradiance is inversely proportional to $\lambda^{4}$ and increases as $a^{6}$, the angular distribution of the scattered light is isotropic.

The angular dependence of the scattered intensity by an ensemble of $N$ randomly distributed particles with mean distance between particles bigger than the wavelength $\lambda$, unpolarized incident light of intensity $I_{0}$, and distant detector position $r$ is described by Rayleigh formula [6-8]

$$
I(r, \theta)=(2 \pi)^{4} \frac{a^{6}}{\lambda^{4} r^{2}}\left(\frac{m^{2}-1}{m^{2}+2}\right)^{2} N \frac{1+\cos ^{2} \theta}{2} I_{0},
$$

where $m=\frac{n_{s}}{n_{0}}$ is the relative index of refraction of scatterers and ground material, $N$ is the number of particles in the scattering volume $V$, i.e., $N=\rho_{p} V$.

In general, light is polarized and presented as two orthogonal linear polarization components of the incident light field in parallel $\left(\vec{E}_{\| i}\right)$ and perpendicular ( $\vec{E}_{\perp i}$ ) to the scattering plane (see Fig. 3.9). Within the detector plane located at a distance $r$ from the origin along the vector $\vec{s}_{1}$, two orthogonal polarization components $\vec{E}_{\| s}$ and $\vec{E}_{\perp s}$ of the scattered light create a specific polarization state depending on amplitudes and phase shift between components.

Figure 3.10 illustrates isotropy of Rayleigh scattering for unpolarized incident light and polarization insensitive detection (curve 3 ) and polarization ability of the scattering particles for the particular direction of detection $\left(90^{\circ}\right.$ and $\left.270^{\circ}\right)$ where only light polarized perpendicular to the scattering plane is scattered (compare curves 1 and 2)). An ideal isotropy (circleshaped phase function) of the scattering is achieved if incident light is linear polarized perpendicular to the scattering plane (curve 1).

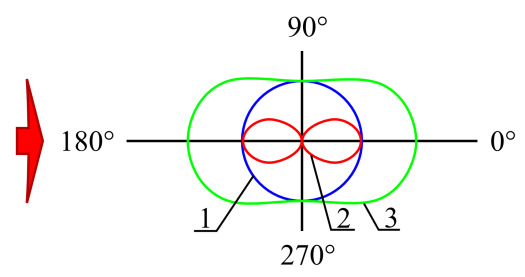

Fig. 3.10 Rayleigh scattering: light distribution in the scattering plane for two orthogonal linear polarization states and unpolarized incident light. 1 - Electric vector perpendicular to the scattering plane; 2 - electric vector parallel to the scattering plane; 3 - unpolarized light

For the NIR light and typical biological scatterers with $m=1.05-1.10$, the maximum particle radius is about $12-14 \mathrm{~nm}$ for the Rayleigh theory to remain valid.

The Rayleigh-Gans or Rayleigh-Debye theory addresses the problem of calculating the scattering by a special class of arbitrary shaped particles, it requires $|m-1|<<1$ and $\frac{2 \pi a^{\prime}}{\lambda}|m-1|<<1$, where $a^{\prime}$ is the largest dimension of the particle [22-24]. These conditions mean that the electric field inside the particle must be close to that of the incident field and the particle can be viewed as a collection of independent dipoles that are all exposed to the same incident field. A biological cell might be modeled as a sphere of cytoplasm with a higher refractive index $\left(n_{c}=1.370\right)$ relative to that of the surrounding interstitial medium $\left(n_{i}=1.350\right)$, then $m$ $=1.015$ and for the NIR light this theory will be valid for the particle dimension up to $a^{\prime}=850-950 \mathrm{~nm}$. This approximation has been applied extensively to 
calculations of light scattering from suspensions of bacteria [24]. It can be applicable for describing of light scattering from cell components (mitochondria, lysosomes, peroxisomes, etc) in tissues due to their small dimensions and refraction.

\subsubsection{Mie scattering}

Mie or Lorenz-Mie scattering theory relates to scattering by comparatively large spherical particles, which are of the order of the wavelength, and based on an exact solution of Maxwell's electromagnetic field equations for a homogeneous sphere [2-25]. Typically tissues contain both types of scatterers small and large (for instance, cell components and collagen fibers of connective tissues, see Fig.3.7). Mie theory operates with the following relevant particle parameters: radius $a$ and complex refractive indices of its material $n_{s}\left(\lambda_{0}\right)$ and dielectric host (ground material) $n_{0}\left(\lambda_{0}\right)$

$$
n_{s, 0}\left(\lambda_{0}\right)=n_{s, 0}^{\prime}\left(\lambda_{0}\right)+i n_{s, 0}^{\prime \prime}\left(\lambda_{0}\right)
$$

where $\lambda_{0}$ is the wavelength in a vacuum. The imaginary part of the complex refractive index of material is responsible for light losses due to absorption. Mie theory yields the scattering ( $Q_{s c a}=\frac{\sigma_{s c a}}{\pi a^{2}}$ ) and absorption $\left(Q_{a b s}=\frac{\sigma_{a b s}}{\pi a^{2}}\right)$ efficiencies and the phase function from which the absorption and scattering cross sections $\left(\sigma_{s c a}\right.$ and $\sigma_{a b s}$ ) and the scattering anisotropy factor $g$ are calculated:

$\sigma_{s c a}^{\text {Mie }}=\frac{\lambda_{0}^{2}}{2 \pi n_{0}^{2}} \sum_{n=1}^{\infty}(2 n+1)\left(\left|a_{n}\right|^{2}+\left|b_{n}\right|^{2}\right)$,

$\sigma_{a b s}^{M i e}=$

$=\frac{\lambda_{0}^{2}}{2 \pi n_{0}^{2}} \sum_{n=1}^{\infty}(2 n+1)\left[\operatorname{Re}\left(a_{n}+b_{n}\right)-\left(\left|a_{n}\right|^{2}+\left|b_{n}\right|^{2}\right)\right]$

$p^{\text {Mie }}(\theta)=\frac{\lambda_{0}^{2}}{2 \pi n_{0}^{2} \sigma_{s c a}^{M i e}}\left(\left|S_{1}\right|^{2}+\left|S_{2}\right|^{2}\right)$,

where $a_{n}$ and $b_{n}$ are Mie coefficients, which are functions of the relative complex refractive index of particles $(m)$ and parameter $\alpha=\frac{2 \pi a n_{0}}{\lambda_{0}}$; an asterisk indicates that the complex conjugate is to be taken;

$$
\begin{aligned}
& a_{n}=\frac{\psi_{n}(\alpha) \psi_{n}^{\prime}(m \alpha)-m \psi_{n}(m \alpha) \psi_{n}^{\prime}(\alpha)}{\zeta(\alpha) \psi_{n}^{\prime}(m \alpha)-m \psi_{n}(m \alpha) \zeta_{n}^{\prime}(\alpha)}, \\
& b_{n}=\frac{m \psi^{\prime}(m \alpha) \psi_{n}(\alpha)-\psi_{n}(m \alpha) \psi_{n}^{\prime}(\alpha)}{m \psi_{n}^{\prime}(m \alpha) \zeta_{n}(\alpha)-\psi_{n}(m \alpha) \zeta_{n}^{\prime}(\alpha)},
\end{aligned}
$$

$\psi_{n}$ and $\zeta_{n}$ are the Riccati-Bessel functions; $S_{1}$ and $S_{2}$ are functions of the polar scattering angle and can be obtained from the Mie theory as

$$
\begin{aligned}
& S_{1}(\theta)=\sum_{n=1}^{\infty} \frac{2 n+1}{n(n+1)}\left\{a_{n} \pi_{n}(\cos \theta)+b_{n} \tau_{n}(\cos \theta)\right\}, \\
& S_{2}(\theta)=\sum_{n=1}^{\infty} \frac{2 n+1}{n(n+1)}\left\{b_{n} \pi_{n}(\cos \theta)+a_{n} \tau_{n}(\cos \theta)\right\},
\end{aligned}
$$

the parameters $\pi_{n}$ and $\tau_{n}$ represent

$$
\begin{aligned}
& \pi_{n}(\cos \theta)=\frac{1}{\sin \theta} P_{n}^{1}(\cos \theta), \\
& \tau_{n}(\cos \theta)=\frac{\mathrm{d}}{\mathrm{d} \theta} P_{n}^{1}(\cos \theta),
\end{aligned}
$$

where $P_{n}^{1}(\cos \theta)$ is the associated Legendre polynomial; the following recursive relationships are used to calculate $\pi_{n}$ and $\tau_{n}$ :

$$
\begin{aligned}
\pi_{n} & =\frac{2 n-1}{n-1} \pi_{n-1} \cos \theta-\frac{n}{n-1} \pi_{n-2}, \\
\tau_{n} & =n \pi_{n} \cos \theta-(n+1) \pi_{n-1},
\end{aligned}
$$

and the initial values are:

$$
\left\{\begin{array}{l}
\pi_{1}=1, \pi_{2}=\cos \theta \\
\tau_{1}=\cos \theta, \tau_{2}=3 \cos 2 \theta
\end{array}\right.
$$

Accounting for definition expressed by Eq. (3.21) scattering anisotropy factor can be calculated from Eq. (3.27)

$$
g^{\text {Mie }}=\frac{\lambda_{0}^{2}}{\pi n_{0}^{2} \sigma_{\text {sca }}^{\text {Mie }}}\left\{\begin{array}{l}
\sum_{n=1}^{\infty} \frac{2 n+1}{n(n+1)} \operatorname{Re}\left(a_{n} b_{n}^{*}\right)+ \\
+\sum_{n=1}^{\infty} \frac{n(n+2)}{n+1} \operatorname{Re}\left(a_{n} a_{n+1}^{*}+b_{n} b_{n+1}^{*}\right)
\end{array}\right\} .
$$

Similarly to the Rayleigh formula (3.23), the angular dependence of the scattered light intensity by an ensemble of $N$ randomly distributed Mie particles with mean distance between particles bigger than the wavelength $\lambda$, for unpolarized incident light of intensity $I_{0}$ and distant detector position $r$, is described by

$$
I(\theta)=p^{M i e}(\theta) \cdot N \cdot I_{0} .
$$

The introduction of the specific scattering and absorption coefficients extrapolated to a volume fraction $f=1$ is useful for describing scattering and absorption properties of an ensemble of Mie scattering particles. In that case and when the particles are sufficiently distant to prevent dependent scattering, the scattering 
coefficient is proportional to the dimensionless volume fraction of scatterers $f$. From Eq. (3.17) it follows:

$$
\mu_{s}=\rho_{p} \sigma_{s c a}=(f / V) \sigma_{s c a}=f \bar{\sigma}_{s c a},
$$

where the specific scattering coefficient $\bar{\sigma}_{s c a}=\sigma_{s c a} / V$ is expressed in $\mathrm{cm}^{-1}, V$ is the unit volume.

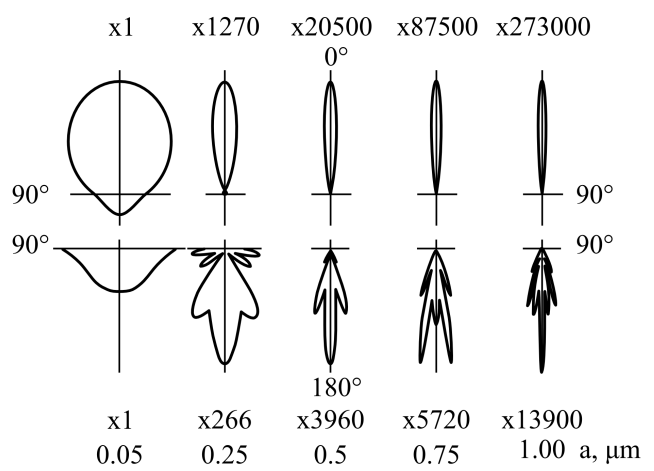

Fig. 3.11 The polar diagrams of the calculated angulardependent intensity distributions (Mie phase functions) for 5 single isotropic spheres with the diameter in the range of those which are typical for cell organelles, from 0.05 to $1.00 \mu \mathrm{m}$ [25].

Mie theory predicts that scattering introduced by spherical micrometer-sized particles is strongest if the particle radius and wavelength are of the same order of magnitude (Figs. 3.11 and 3.12). The scattering coefficient increases strongly with the elevation of the relative index of refraction. In contrast, for the matched refractive indices of scatterers and background material, the scattering coefficient goes to zero, which means that only absorption is responsible now for the light beam extinction [see Eq. (3.18)]. However, because of very low absorption of many tissues in the NIR range, refractive index matching conditions due to perfusion of endogenous or exogenous immersion agents may have a significant influence on tissue optical properties. It is also of great importance that scattering anisotropy factor aspires to 1 (extremely high scattering directness).

In Fig. 3.12, the wavelength dependencies of scattering parameters are also shown. If particle diameter $2 a$ and relative index $m$ are fixed, the wavelength dependencies are caused by variation of the ratio of particle diameter and wavelength only. The spectral variation of the relative index $m$ has been neglected in these calculations, but may be relevant in practice.

For example, epithelial cell nucleus can be considered as a spherical Mie scatter with refractive index, $n_{n c}$, which is higher than that of the surrounding cytoplasm, $n_{c p}$, i.e., $m=n_{n c} / n_{c p}$. Normal nuclei have a characteristic diameter $2 a=4-7 \mu \mathrm{m}$. In contrast, dysplastic nuclei can be as large as $20 \mu \mathrm{m}$, occupying almost the entire cell volume. In the visible and NIR range, where the wavelength $\lambda<<2 a$, Mie theory can be well approximated by the Van de Hulst approximation or also called anomalous diffraction approximation, which is applicable for optically soft particles $[(m-1)$ $<<1$ ] [26]. Thus, the elastic scattering cross section of the cell nuclei can be described as

$$
\sigma_{s c a}(\lambda, a)=2 \pi a^{2}\left(1-\frac{2 \sin \delta}{\delta}+\left(\frac{2 \sin \delta}{\delta}\right)^{2}\right)
$$

where $\delta=\frac{4 \pi a(m-1)}{\lambda_{0}}, \lambda_{0}$ is the wavelength of the light in free space. The cross section varies periodically with inverse wavelength and gives rise to a periodic component in the tissue optical reflectance. Since the frequency of this variation (in inverse wavelength space) is proportional to particle size, the nuclear size distribution can be obtained from the Fourier transform of the periodic component.

Mie theory is strictly applicable only to particles of particular regular shapes, but results are still useful if the shape is irregular. The oscillatory structure of the scattering coefficient and anisotropy factor as a function of particle size, which is observed with spherical particles (see Figs. 3.12a,b), will be averaged out in the case of irregular shape.

Actual biological tissue models are more complex than a monodispersive system of distant spherical particles or even randomly shaped ones. Sometimes, a mixture of large particles contributing high scattering anisotropy and small particles with increased scattering towards shorter wavelengths may be a good approximation to describe tissue scattering properties.

Besides theoretical Mie phase function [see Eq. (3.27)], several semi-empirical approximations for the scattering phase function have been used in tissue photonics $[2-5,18,28]$. One of the most often exploited is the Henyey-Greenstein (HG) phase function

$$
p^{H G}(\theta)=\frac{1}{4 \pi} \cdot \frac{1-g^{2}}{\left(1+g^{2}-2 g \cos \theta\right)^{3 / 2}}
$$

The HG phase function has one parameter $g$ that may be represented as the infinite series of Legendre polynomials $P_{n}^{1}(\cos \theta)$,

$$
p^{H G}(\theta)=\frac{1}{4 \pi} \sum_{n=0}^{\infty}(2 n+1) f_{n} P_{n}^{1}(\cos \theta)
$$

where $f_{n}=g^{n}$ is the $n^{\text {th }}$ order moment of the phase function.

Figure 3.13 illustrates behavior of the HenyeyGreenstein scattering phase function calculated for $g$ values characteristic for hard (0.6-0.9) and soft (0.8$0.95)$ tissues, as well as for highly anisotropic scattering systems such as blood (0.995). 


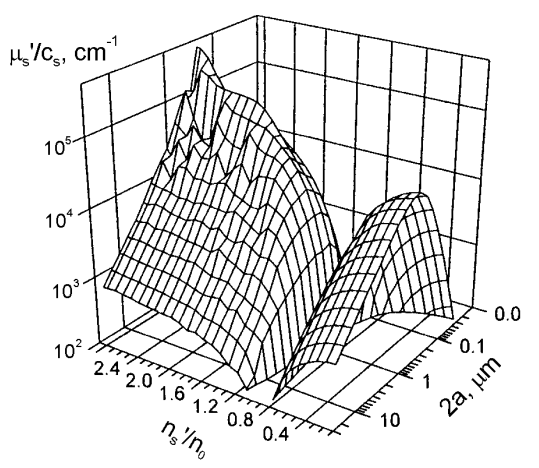

$\mathrm{a}$

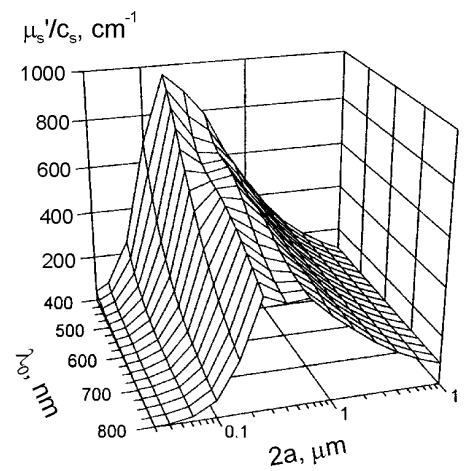

c

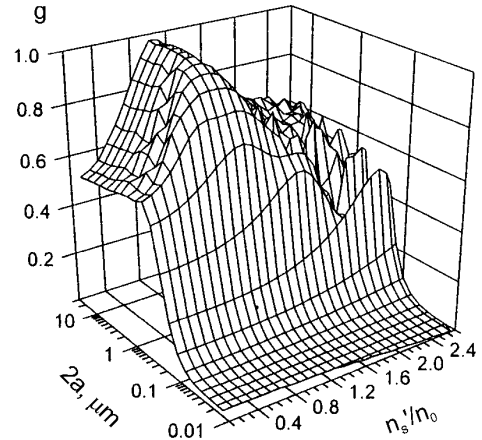

b

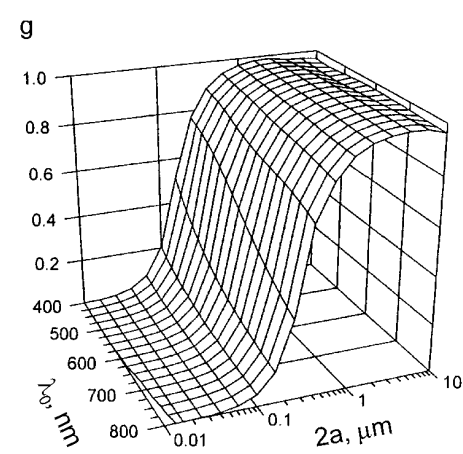

d

Fig, 3.12 Mie theory: the scattering properties [normalized reduced scattering coefficient $\mu_{\mathrm{s}}^{\prime} / C_{\mathrm{s}}=\left(\mu_{\mathrm{s}} / C_{\mathrm{s}}\right)(1-g)(\mathrm{a})$ and scattering anisotropy factor $g(b)]$ of an ensemble of noninteracting nonabsorbing spherical particles calculated at 633 $\mathrm{nm}$ for the broad ranges of diameter $(2 a)$ and relative index of refraction $\left(m=n_{s}{ }^{\prime} / n_{0}\right)$ which are characteristic to tissue and cellular structure components; wavelength-diameter dependences of $\mu_{\mathrm{s}}^{\prime}\left(\lambda_{0}, 2 a\right) / C_{\mathrm{s}}$ (c) and $g\left(\lambda_{0}, 2 a\right)$ (d) at relative index of refraction $m=1.07$, typical for normal soft tissues; $C_{\mathrm{s}}$ is the volume fraction of the particles [5, 27].

\subsubsection{Multiple scattering}

Parameters introduced are related to light tissue interaction on the level of a single scattering that occurs when a wave undertakes no more than one collision with particles of the medium in which it propagates. For example, this is a case for healthy transparent front human eye tissues, such as cornea, eye lens and vitreous body (see Fig. 2.6) [1], or for a tissue slice which is sufficiently thin that single scattering approximation accurately estimates the reflection and transmission of the slab. In contrast, in many real situations, especially in in vivo studies of skin, breast, brain and etc., a multiple scattering - a scattering process, in which on average each photon undertakes many scattering events, is more likely [2-5, 9-38].

To evaluate what kind of scattering regime is realized a so called mean free path length (MFP) may be introduced. MFP is the mean distance between two successive interactions with scattering or absorption which a photon travelling in a scattering-absorption medium experiencing,

$$
\mathrm{MFP}=l_{p h}=1 / \mu_{t}
$$

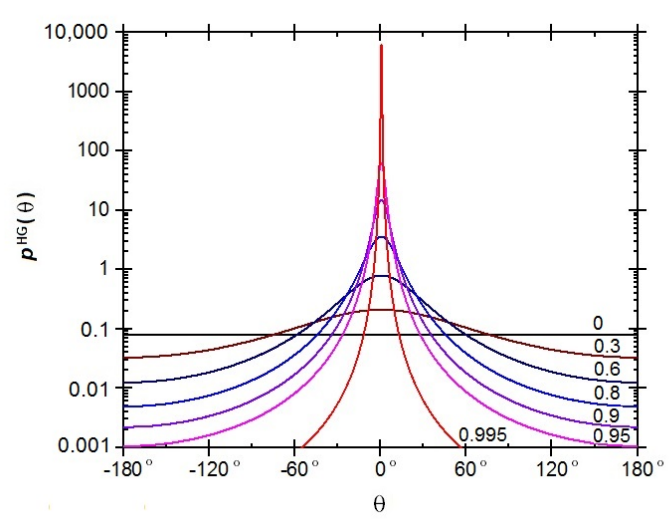

Fig. 3.13 The Henyey-Greenstein (HG) scattering phase function often used in tissue photonics; it is calculated for typical $g$ values characteristic for hard (0.6-0.9) and soft (0.8-0.95) tissues and blood (0.995). 
Thus, one can differentiate optically thin (transparent) or optically thick sample is under investigation. "Optical thickness" is the depth of a material or medium in which the intensity of light of a given wavelength is reduced by a factor of 1/e because of absorption and/or scattering [see Eq. (3.18)]. A sample of high physical thickness and/or high turbidity that correspond to a few "optical thicknesses" is optically thick, as well as a sample with a low thickness and/or low turbidity that correspond to one or less than one "optical thickness" is optically thin.

The radiation transfer theory (RTT) is the basic theory allowing one to calculate light distributions in the multiple scattering media with absorption, such as tissues. The heart of this theory is the radiation transfer equation (RTE) - the Boltzmann or linear transport equation, which is a balance equation describing the flow of particles (e.g., photons) in a given volume element that takes into account their velocity $c$, location $r$, and changes due to collisions (i.e., scattering and absorption). The basic parameter for this theory is the reduced scattering coefficient that is incorporating the scattering coefficient $\mu_{\mathrm{s}}$ and the scattering anisotropy factor $g$ as the following expression

$$
\mu_{s}^{\prime}=\mu_{s}(1-g)
$$

or at usage of dimensionless volume fraction of scatterers $f$ and the specific scattering coefficients $\bar{\sigma}_{\text {sca }}$ [see Eq. (3.35)] as

$$
\mu_{s}^{\prime}=f \bar{\sigma}_{s c a}\left[1-g\left(\lambda_{0}, 2 a\right)\right]
$$

The transport mean free path (TMFP) of a photon (cm) is defined as

$$
l_{t r}=\frac{1}{\mu_{a}+\mu_{s}^{\prime}}
$$

where $\mu_{a}+\mu_{s}^{\prime}=\mu_{t r}$ is the photon transport coefficient. The TMFP in a medium with anisotropic single scattering significantly exceeds the MFP, $l_{t r}>>l_{p h}$. The $l_{t r}$ is the distance over which the photon loses its initial direction.

In tissues, at visible and NIR light propagation, reduced scattering coefficient $\mu_{s}{ }^{\prime}$ mostly defines light transport, because absorption much lower than scattering, $\mu_{s}{ }^{\prime}>>\mu_{a}$,

$$
l_{t r} \cong \frac{1}{\mu_{s}^{\prime}}
$$

The reduced scattering coefficient $\mu_{s}{ }^{\prime}$ describes the diffusion of photons in a random walk of step size of $\frac{1}{\mu_{s}^{\prime}}[\mathrm{cm}]$, where each step involves isotropic scattering. Schematically scattering processes are presented in Fig. 3.14 [20]. This is equivalent to description of photon movement using many small steps $\frac{1}{\mu_{s}}$ that each involve only a partial (anisotropic) deflection angle if there are many scattering events before an absorption event, i.e., $\mu_{s}{ }^{\prime}>\mu_{a}$ (diffusion regime). Parameter $\mu_{\mathrm{s}}{ }^{\prime}$ is useful in the diffusion regime. Figure 3.14 shows the equivalence of taking 10 smaller steps of $\mathrm{MFP} \cong \frac{1}{\mu_{s}}$ with anisotropic deflection angles and one big step with a TMFP $\cong \frac{1}{\mu_{s}^{\prime}}$. Diffusion regime is well described analytically in the framework of diffusion approximation of the radiation transfer equation diffusion theory.

A power law for dependence of the reduced scattering coefficient on the wavelength is typical for many tissues, $\mu^{\prime}{ }_{s}(\lambda)=\mu^{\prime}{ }_{s}\left(\lambda_{r e f}\right)\left(\lambda / \lambda_{r e f}\right)^{-b}$. In Refs. [18, $20,21], \lambda_{r e f}=500 \mathrm{~nm}$. For different tissues and tissue conditions (normal or pathological, coagulated or dehydrated, etc.) parameter $b$ is ranging from 0.5 to 1.6 for the visible-NIR light scattering (600-2000 nm).

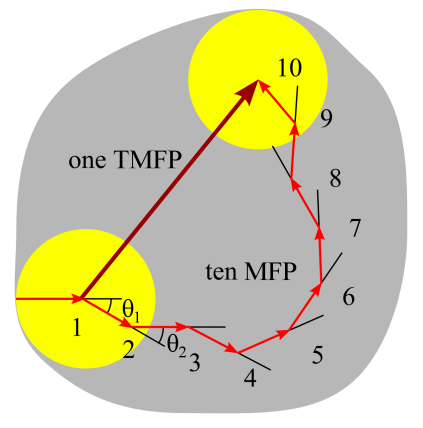

a

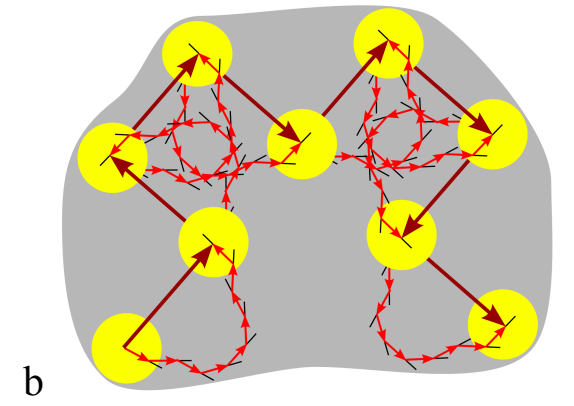

Fig. 3.14 Multiple scattering: mean free path (MFP) and transport MFP (TMFP) definitions; $\mathrm{MFP} \cong 1 / \mu_{s}\left(\mu_{s}>>\right.$ $\left.\mu_{a}\right), \mathrm{TMFP} \cong 1 / \mu_{s}{ }^{\prime}\left(\mu_{s}{ }^{\prime}>>\mu_{a}\right)$, and $<\cos \theta>=0.90$, i.e., $<\theta>\approx \theta_{1} \approx \theta_{2} \approx \ldots \approx \theta_{10} \approx 26^{\circ}$ and $\mu_{s}{ }^{\prime}=\mu_{s}(1-\mathrm{g})=0.10 \mu_{s}$ (a); illustration of photon random walk of TMFP-step size; scattering particles are shown as yellowish disks, small arrows show direction of photon migration between consecutive elementary interaction acts with 
scattering; big arrows show direction of effective photon migration as a result of multiple scattering; for more anisotropic phase function (bigger $g$-factor) more elementary scattering steps needed to transfer photon distribution to isotropic one (b); http://omlc.ogi.edu/classroom/ece532/class3/musp.html.

For example, for tissue model of noninteracting Mie scatterers of equal diameter $2 a$, for typical parameters of soft tissues $(g>0.9 ; 5<2 \pi a / \lambda<50 ; 1<m<1.1)$ the reduced scattering coefficient is described by a simple expression [34]

$$
\mu_{s}^{\prime}=3.28 \pi a^{2} \rho_{p}\left(\frac{2 \pi a}{\lambda}\right)^{0.37} \times(m-1)^{2.09}
$$

Attenuation of a wide (expanded) laser beam of intensity $I_{0}$ in a thick tissue at depths $z>l_{d}=\frac{1}{\mu_{\text {eff }}}$, where diffusion regime takes place, is described as [5]

$$
I(z) \approx I_{0} b_{s} \exp \left(-\mu_{e f f} z\right)
$$

where $\mu_{e f f}=\sqrt{3 \mu_{a}\left(\mu_{s}^{\prime}+\mu_{a}\right)}$, which follows from the diffusion theory; $b_{s}$ accounts for additional irradiation of the upper layers of a tissue due to backscattering (photon redistribution or recycling effect). Respectively, the depth of light penetration into a tissue is

$$
l_{e}=l_{d}\left[\ln b_{s}+1\right] \text {. }
$$

Typically, for soft tissues $b_{s}=1-5$ for beam diameter of 1-20 mm. Thus, when wide laser beams are used for irradiation highly scattering tissues with low absorption, $\mathrm{CW}$ light energy is accumulated in tissue due to high multiplicity of random long-path photon migrations. A highly scattering medium works as an optical cavity with random reflectors (scatterers) providing the capacity of light energy. The light power density within the superficial tissue layers may substantially (up to fivefold) exceed the incident power density and cause the over dosage during photodynamic therapy or overheating at interstitial laser thermotherapy. On the other hand, photon recycling effect can be used for more effective irradiation of under surface lesions at relatively small incident power densities.

To describe pulsed light propagation, which is often used in tissue spectroscopy and imaging, in a tissue the time-dependent radiation transfer theory should be used [2-5, 35-38]. This theory is based on the time-dependent linear transport equation, which is a balance equation describing the time-dependent flow of particles (e.g., photons) in a given volume element that takes into account their velocity $c$, location $\bar{r}$, and changes due to collisions (i.e., scattering and absorption).
The time-resolved methods that use pulsed or intensity modulated laser beams for irradiating of tissues under study can separate different components of scattering photons from a sample in forward (transillumination) or backscattering operating modes. One of these groups of photons is a so called ballistic (coherent) photon group which is consists of unscattered and strictly straightforward scattered photons. The other group is composed of quasi-ballistic (snake or zigzag) photons which are photons that migrate within a scattering medium along trajectories that are close but not the same as for ballistic photons. To the third group the so called diffusion photons belong. This is typically the largest group of photons that migrated for longer time in a tissue along multi-step random trajectories. Each of these groups carries information about optical (morphological) properties of a tissue. Ballistic photons are good for getting precise tissue images similar to $\mathrm{x}$ ray computer tomography however in many tissues because of strong scattering this group of photon is typically negligibly small. The snake photons, having undergone only a few scattering events, all of which are in the forward or near-forward direction, retain the image bearing characteristics to some extent, however their amount is rather big in comparison with the ballistic photons, thus they are detectable and provide good spatial resolution. In contrast, due to high intensity of diffusion component there is much more practical to use diffusion photons to estimate optical properties of tissues however a spatial resolution could not be very high.

Figure 3.15 illustrates short pulse propagation in tissues, where an ultrashort laser pulse incident on tissue surface spreads in bulk into three main groups of photons - first arriving to detector (ballistic), more intensive group of snake photons, and a large group of diffuse late arriving photons with a broad distribution. Figure $3.15 \mathrm{~b}$ demonstrates how decays the amplitude of intensity modulated light at frequency $\omega=2 \pi \mathrm{V}$ propagating through a scattering tissue and how shifts its modulation phase, $\Delta \Phi$. For a weakly absorbing scattering medium, when $\mu_{a}<\frac{\omega}{c}$ and $<\mu^{\prime}{ }_{s}$, an alternating component of the scattered light is a outgoing spherical intensity (or photon density) wave with the center at the point $\bar{r}=0$ which oscillates at a modulation frequency $\omega$ with the modulation depth [5]

$$
m_{U}(\bar{r}, \omega)=m_{I} \cdot \exp \left(\bar{r} \sqrt{\frac{\mu_{a}}{D}}\right) \cdot \exp \left(-\bar{r} \sqrt{\frac{\omega}{2 c D}}\right)
$$

and undergoes a phase shift relative to the phase value at point $\bar{r}=0$ equal to

$$
\Delta \Phi(\bar{r}, \omega) \cong \bar{r} \sqrt{\frac{\omega}{2 c D}},
$$


where $m_{I}=\frac{\delta I}{I_{0}}$ is the modulation depth of the incident light with mean intensity $I_{0}$ and alternating amplitude $\delta I$;

$$
D=\frac{1}{3\left(\mu_{s}{ }^{\prime}+\mu_{a}\right)} \cong \frac{1}{3 \mu_{s}{ }^{\prime}}
$$

is the photon diffusion coefficient, $\mathrm{c}^{-1}$. Accounting for Eq. (3.49) expressions (3.47) and (3.48) could be further simplified

$$
\begin{aligned}
& m_{U}(\bar{r}, \omega)= \\
& =m_{I} \cdot \exp \left(\bar{r} \sqrt{3 \mu_{a} \mu_{s}^{\prime}}\right) \cdot \exp \left(-\bar{r} \sqrt{\frac{3 \omega \mu_{s}{ }^{\prime}}{2 c}}\right), \\
& \Delta \Phi(\bar{r}, \omega) \cong \bar{r} \sqrt{\frac{3 \omega \mu_{s}^{\prime}}{2 c}} .
\end{aligned}
$$
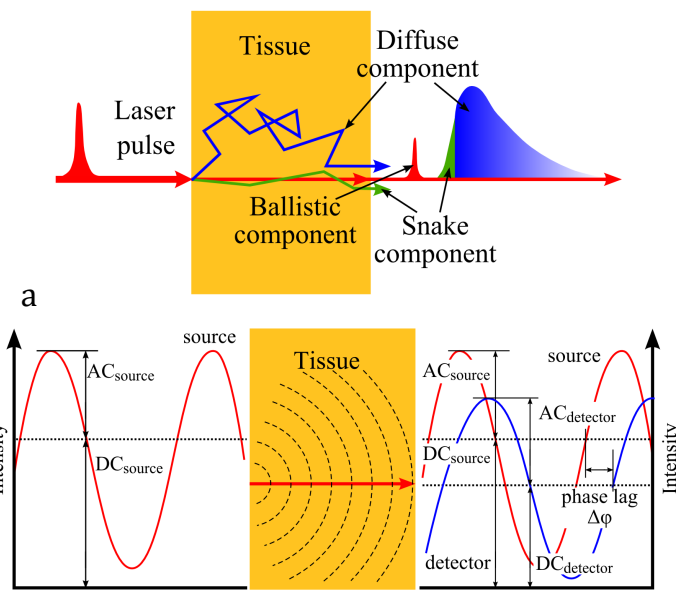

b
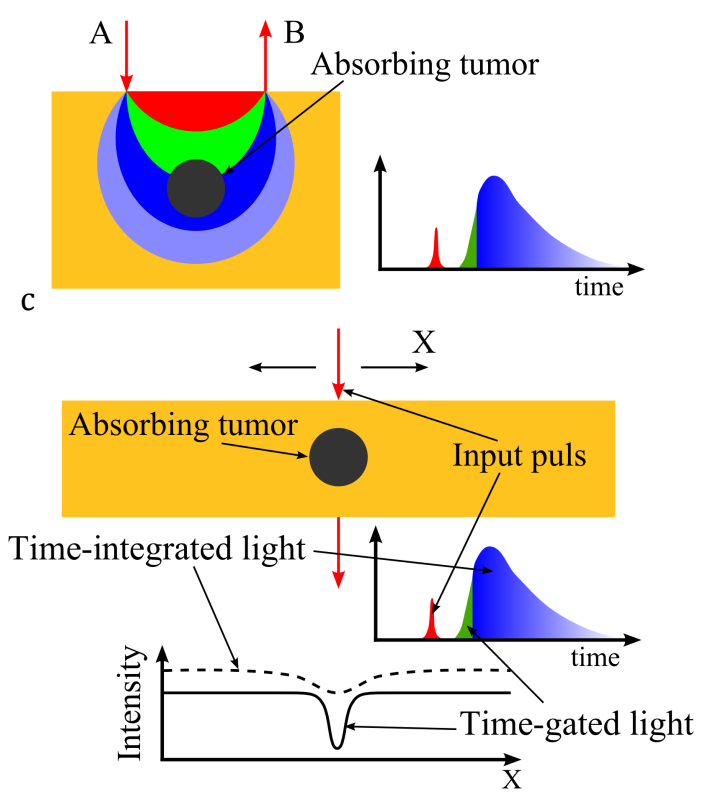

$\mathrm{d}$
Fig. 3.15 Short pulse and intensity modulated light propagation in tissues: (a) an ultrashort laser pulse spreads into three main groups of photons - ballistic (first arriving to detector), snake, and diffuse (broad distribution of late arriving photons); (b) illustration of intensity modulation amplitude decay and phase shift due to scattering; (c) illustration of two typical geometries for optical imaging of absorbing inhomogeneity (tumor) in tissues, A - probing beam (typically delivered by a fiber), B - detected radiation (typically collected by a detector fiber), left transillumination mode, right - backscattering mode; (d) illustration of absorbing inhomogeneity (tumor) image contrast enhancement due to time-gating by selecting early arriving (snake) photons only, $\mathrm{X}$ is the light beam scanning axis [35].

To generate and support a photon density wave a light scattering medium is needed. However, high scattering turns a photon density wave to decay with distance (see Eq. (3.50)). Measuring $m_{U}(\bar{r}, \omega)$ and/or $\Delta \Phi(\bar{r}, \omega)$ allows one to determine separately the reduced scattering coefficient $\mu_{s}{ }^{\prime}$ and the absorption coefficient $\mu_{a}$, and evaluate the spatial distributions of these parameters needed for imaging of tissue pathologies. For biomedical applications, in particular, optical mammography or brain imaging, typical modulation frequencies are lying in the range $\omega / 2 \pi=$ 50-1000 MHz [2-5, 35-38].

Two typical geometries (transillumination and backscattering) for a time-resolved optical imaging of absorbing inhomogeneity (tumor) in tissues are shown (see Fig. 3.15c). Figure 3.15d illustrates image contrast (spatial resolution) enhancement due to time-gating by selecting early arriving (snake) photons at pulse excitation.

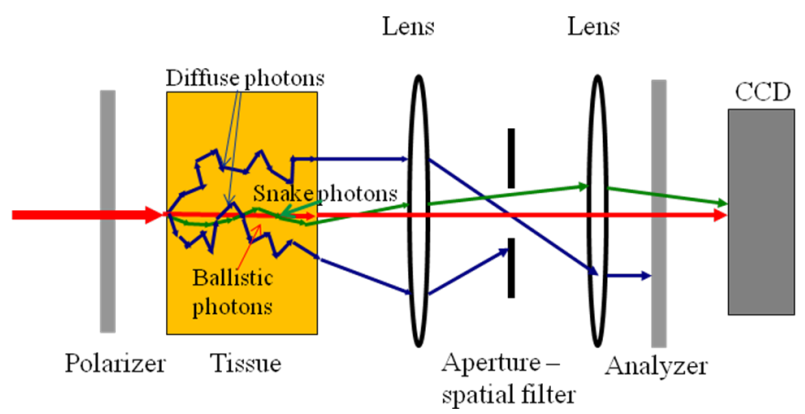

Fig. 3.16 Schematic representation of spatial filtering using an aperture in the detector space and polarization gating with a linear polarizer in the light incidence space and in-line polarized analyzer in the detector space; diffuse photons are not detected due to their high deflection angles (spatial filtering) or due to loosing initial polarization state because of long travelling paths.

To improve spatial resolution of diffusion methods various approaches for selective detection of informative photons were suggested, such as spatially- 
resolved, angular-resolve, and polarization-sensitive gating. Spatial filtering and polarization gating approaches are shown schematically in Fig. 3.16. It is well seen that diffuse photons are not detectable due to their high deflection angles (spatial filtering) or due to loosing initial polarization state because of long travelling paths within tissue. Thus more contrast image could be produced by detection pure ballistic and leastscattering (snake) photons which have minimal deflection and alternation of polarization state.

The most complete information about tissue optical properties could be obtained when spectrum of the scattered light is measured in the wavelength range of interest. This spectrum may be differential, measured or calculated for a certain scattering angle, or integrated within an angle (field of view) of the measuring spectrometer. In general, it includes transmitted and backscattered (back reflected) light within a narrow field of view, such as measured in geometry of collimated transmittance or specular reflectance, or totally forward or backward scattered light measured using integrating sphere technique. Figure 3.17 illustrates typical spectral measurement geometries used for in vitro and in vivo studies [2-5, 17, 18]. In vitro measurements include angular spectral dependences $I(\theta$, $\lambda$ ) using goniometric detection with the narrow slit in front of a detector, and angular integrated spectra by integrating sphere. Angular measurements $[I(\theta, \lambda)]$ are used for evaluation of phase scattering function $p(\theta, \lambda)$ [see Eqs. (3.27) and (3.37)] for the particular wavelength. Sometimes backward $\left[I_{b}(\theta, \lambda)\right]$ and forward $\left[I_{f}(\theta, \lambda)\right)$ scattering intensities, or collimated transmitted $\left[I_{c}(\lambda)\right]$ and specular (Fresnel) reflected $\left[I_{F}(\lambda)\right]$ intensities are measured to characterize tissue sample optical properties. The collimated transmittance and specular (Fresnel) reflectance spectra are introduced as

$$
\begin{aligned}
& T_{c}(\lambda)=\frac{I_{c}(\lambda)}{I_{0}(\lambda)}, \\
& R_{F}(\lambda)=\frac{I_{F}(\lambda)}{I_{0}(\lambda)} .
\end{aligned}
$$

The total transmittance $\left[T_{t}(\lambda)\right]$, and diffuse reflectance $\left[R_{d}(\lambda)\right]$ spectra measured with the help of integrating spheres are introduced as

$$
\begin{aligned}
& T_{t}(\lambda)=\frac{I_{t}(\lambda)}{I_{0}(\lambda)}=T_{c}(\lambda)+T_{d}(\lambda), \\
& I_{t}(\lambda)=I_{c}(\lambda)+\int_{-\frac{\pi}{2}}^{\frac{\pi}{2}} I_{f}(\theta, \lambda) \mathrm{d} \theta ;
\end{aligned}
$$

$$
\begin{aligned}
& R_{d}(\lambda)=\frac{I_{b}(\lambda)}{I_{0}(\lambda)}, \\
& I_{b}(\lambda)=\int_{\frac{\pi}{2}}^{-\frac{\pi}{2}} I_{b}(\theta, \lambda) \mathrm{d} \theta .
\end{aligned}
$$

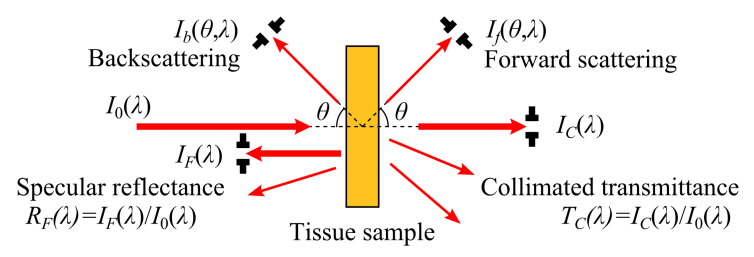

a

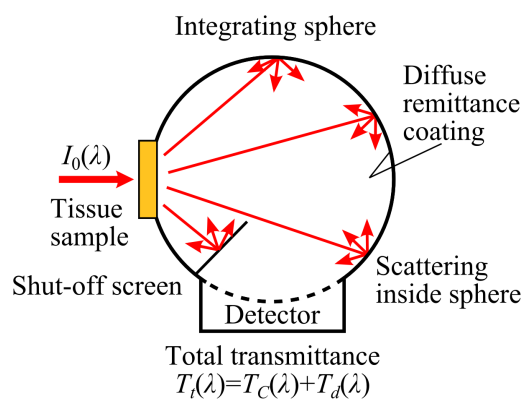

$\mathrm{b}$
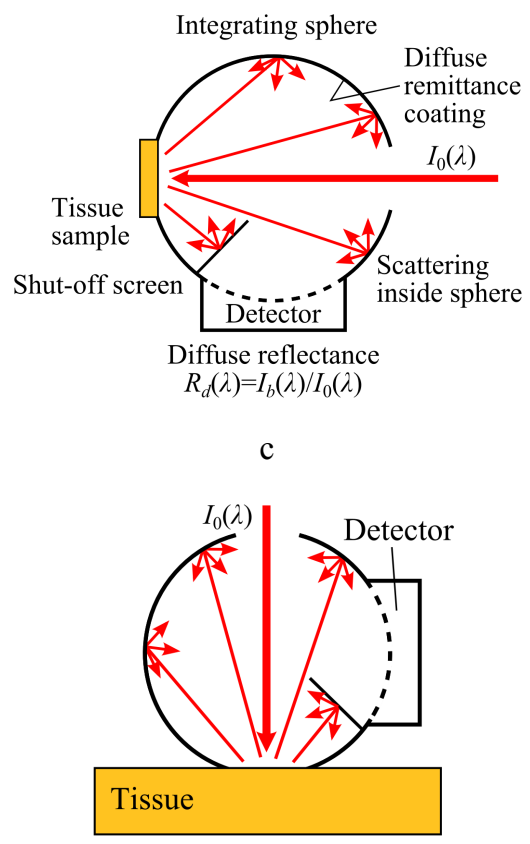

d

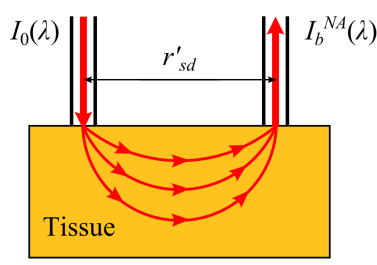


e

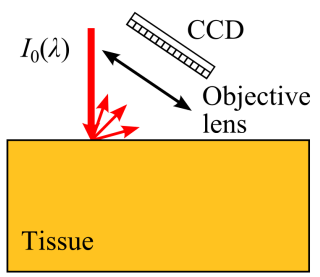

f

Fig. 3.17 Typical geometries used for in vitro and in vivo spectral measurements: in vitro goniometric detection with the narrow slit in front of a detector $I(\theta$, $\lambda$ ) (phase scattering function $p(\theta, \lambda)$ measurements) and fixed angle detection (collimated transmittance $T_{c}(\lambda)(\theta$ $=0)$ and specular reflectance $R_{F}(\lambda)(\theta=2 \pi)(\mathrm{a})$; in vitro angular integrated detection using an integrating sphere: the total transmittance $T_{t}(\lambda)$ (b) and diffuse reflectance $R_{d}(\lambda)(\mathrm{c})$; in vivo integrating sphere detection (d), spatially-resolved fiber (e) or CCD (f); the integrating surface of the sphere is coated with non-fluorescing material $\left(\mathrm{BaSO}_{4}\right.$, or $\mathrm{MgO}$, or Spectrolan $\left.{ }^{\circledR}\right)$, which have nearly $100 \%$ diffuse remittance over the entire optical and NIR spectrum of interest; shut-off screen prevents direct irradiation of the detector; the intensity of light entered into a sphere is measured as the signal of the detector multiplied by the ratio of areas of the entire sphere and detector surfaces; the accuracy of such measurements evidently depends on this ratio.

In vivo studies could be done in different ways, but the most typical geometries are the spatially-resolved backreflectance using two fibers with numerical aperture $N A$ - one as a light source $\left[I_{0}(\lambda)\right]$ and another as a detector $\left[I_{b}^{N A}(\lambda)\right]$ with variable source-detector separation $r_{s d}$ (Fig. 3.17d), or using CCD (CMOS) camera with objective lens (Fig. 3.17e). Integrating sphere measurements of diffuse reflectance are also often provided (Fig. 3.17d).

The modeling of light propagation in a tissue by taking into account the experimental geometry, light source and detector characteristics, known optical properties of a sample, and predicted measurement and associated accuracies is often need for planning of strategy of light treatment or diagnosis. Such modeling and predictions are classified as a solution of the forward scattering problem. In contrast, the inverse scattering problem solution is the attempt to take a set of measurements and error estimates, and only a limited set of parameters describing the sample and experimental specificity, and to derive the remaining parameters. Usually the geometry is known, intensities or their parameters are measured, and the optical properties or sizes and refractive indices of tissue scatterers need to be derived. If these properties are considered to be spatially varying, then the resultant solutions can be presented as a $2 \mathrm{D}$ or $3 \mathrm{D}$ function of space, i.e., as an image.

\subsection{Scattering of the coherent light-speckles}

3.3.1 Random phase screen concept and speckle formation

When coherent (laser) light is reflected from a rough surface or passes through a bulk scattering medium, speckle patterns are produced as a result of interference of a large number of elementary waves (wavelets) with random phases that arise due to light interaction with random scatterers [5, 9, 39-61]. Figure 3.18 illustrates this for a He-Ne laser beam reflection by a rough surface, where complex interference of the scattered coherent wavelets appears as an interference pattern with the spatially resolved intensity spots. In contrast, at laser beam transportation through a bulk tissue (human finger) due to multiple scattering beam is transformed to diffuse radiation, speckle size became comparable with the wavelength and could not be seen by a naked eye. This situation is often considered as a total loosing of light coherence. However at multiple scattering coherent properties of light also could be detected and used for tissue functional diagnostics. A single-mode fiber having a core diameter comparable with the wavelength allows for detection a single or a small group of neighboring speckles in the pattern. Speckle temporal dynamics gives information about intensity fluctuations connected with Brownian motion of biological molecules or flow of the blood particles. This is the basis for diffusion wave spectroscopy (DWS) or diffusion wave imaging (DWI) $[5,52,53,61]$.

Generally, there are two types of speckles: subjective speckles, which are produced in the image space of an optical system (including an eye), and objective speckles, which are formed in a free space and are usually observed on a screen (CCD) placed at a certain distance from an object. Since the majority of tissues are optically non-uniform, their irradiation with a coherent light always gives rise to the appearance of speckle patterns, which either distort the results of measurements and, therefore, should be eliminated by statistical averaging, or provide a new information about the structure and the motion of a tissue and its constituents $[5,48]$. The average size of a speckle in the far-field zone is estimated as

$$
d_{a v} \approx \frac{\lambda}{\varphi},
$$

where $\lambda$ is the wavelength and $\varphi$ is the angle of observation.

Figure 3.19 schematically illustrates the principles of the formation and propagation of speckles, shows transmittance and reflectance modes of their observation as a spatial $(x)$ or temporal $(t)$ intensity modulation in the detector's plane (CCD). The incident coherent light at interaction with a random (scattering) medium is 


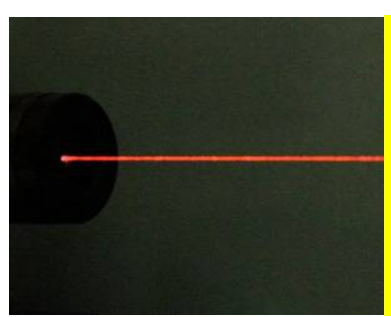

a

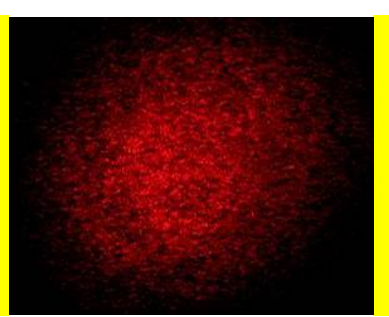

b

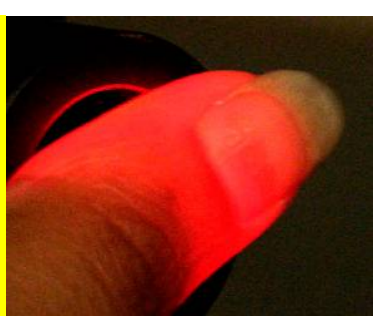

$\mathrm{c}$

Fig. 3.18 Coherent light interaction with inhomogeneous media: (a) Helium-Neon laser $(\lambda=632.8 \mathrm{~nm})$ beam as it travelling in a free space (open air), no interaction, therefore no additional divergence, beam diameter is of $\sim 1 \mathrm{~mm}$; (b) the speckle structure (pattern) formed at laser beam reflection from a rough surface - illustrates coherent properties of light in the form of complex interference of the scattered coherent wavelets; (c) at laser beam transportation through a bulk tissue (human finger) due to multiple scattering it is transformed to diffuse radiation, speckle size became comparable with the wavelength and could not be seen by a naked eye. Sometimes it is saying that coherent light has lost its coherence. However in many experiments coherent properties of incident light could be seen and used even at multiple scattering. For example, at detection with the help of a single-mode fiber a single or small group of neighboring speckles can be selected, dynamics of which give information about intensity fluctuations connected with Brownian motion of biological molecules or flow of blood particles. This is the basis of diffusion wave spectroscopy (DWS). These photos are done by Joel Mobley.

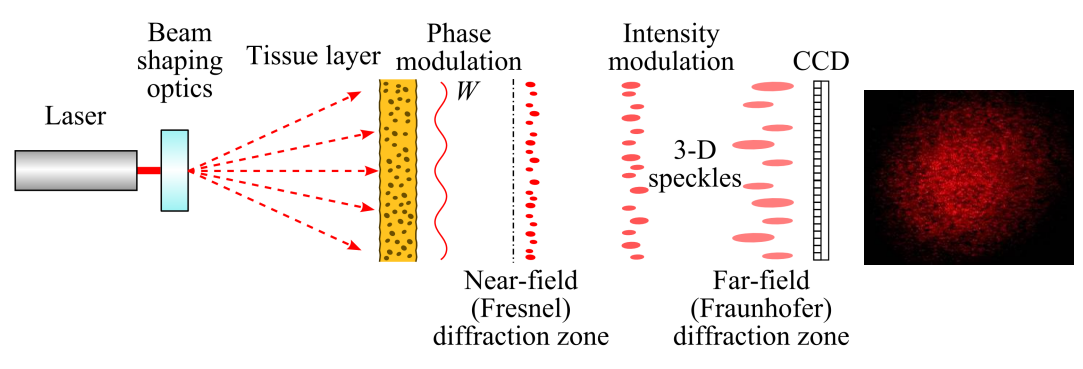

a

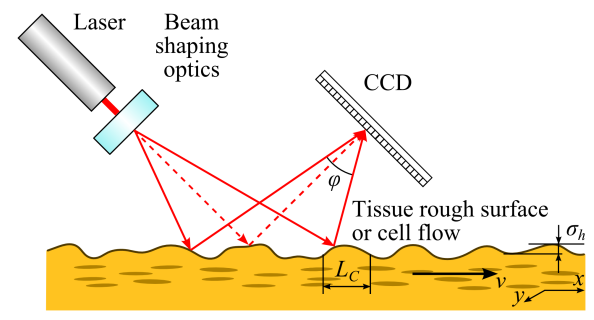

$\mathrm{b}$

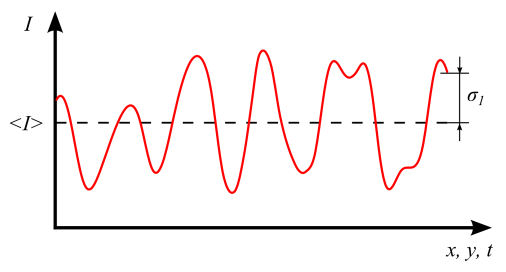

$\mathrm{c}$

Fig. 3.19 Formation and propagation of speckles: observation of speckles in transmittance mode (a); observation of speckles in reflectance mode $(b)$, and spatial $(x, y)$ or temporal $(t)$ intensity modulation in the detector's plane (CCD) (c).

firstly phase modulated with the appearance of the wavelets $(W)$ due to diffraction phenomenon, this modulation is further transformed to intensity modulation as light propagates through near-field (Fresnel) zone to far-field (Fraunhofer) zone of diffraction, where typically speckles are detected. Spatial intensity modulation of the scattered light from a stationary object is registered as alterations of signals detected by the different pixels or group of pixels of a CCD. Temporal intensity modulation is registered at laser beam scanning upon a stationary tissue rough surface or when motion of cells (or other scattering particles) exists. These fluctuations are characterized by the mean value of the intensity $\langle I\rangle$ and the standard deviation $\sigma_{I}$ (see Fig. 3.19c). The object itself is characterized by the standard deviation $\sigma_{h}$ of the altitudes (depths) of inhomogeneities and the correlation length $L_{c}$ of these inhomogeneities (random relief).

Since many tissues and cells are phase objects, for which only refractive index variations are important on the background of negligible absorption, the propagation of coherent beams in tissues can be described on the basis of a random phase screen (RPS) 
model. The amplitude transmission coefficient of an RPS is given by [5, 9, 40-42, 48, 56-59]

$$
t_{s}(x, y)=t_{0} \exp \{-i \Phi(x, y)\}
$$

where $t_{0}$ is the spatially independent amplitude transmission; $\Phi(x, y)$ is the random phase shift introduced by the RPS at the $(x, y)$ point. Such spatial phase fluctuations may be due to variations in the refractive index $n(x, y)$ or the RPS thickness $h(x, y)$ from point to point. For thin transmitting and reflecting RPSs, we have

$$
\begin{aligned}
& \Phi_{t}(x, y)=\left(\frac{2 \pi}{\lambda}\right)[n(x, y)-1] h(x, y), \\
& \Phi_{r}(x, y)=\frac{4 \pi}{\lambda} \cdot h(x, y),
\end{aligned}
$$

respectively. Phase fluctuations of the scattered field are characterized by the standard deviation $\sigma_{\phi}$ and the correlation length $L_{\phi}$. Generally, there are two types of RPSs: weakly scattering RPSs $\left(\sigma_{\phi}^{2}<<1\right)$ and deep RPSs $\left(\sigma_{\phi}^{2}>>1\right)$.

Ideal conditions for formation of speckles, when completely developed speckles arise, can be formulated as the following. Coherent light irradiates a diffusive surface (or a transparency) characterized by Gaussian variations of the optical path $\Delta l(x, y)=\Delta[n(x, y) h(x, y)]$, where $n(x, y)$ is the profile of the index of refraction and $h(x, y)$ is the profile of height (thickness), with standard deviation of the optical path variations, $\sigma_{l}>>\lambda$. Both the coherence length of light and sizes of the scattering area considerably exceed the differences in optical paths due to the surface relief, and many scattering centers contribute to the resulting speckle pattern.

Statistical properties of speckles can be divided into statistics of the first and second orders. Statistics of the first order describes the properties of speckle fields at each point. Such a description usually employs the intensity probability density distribution function $p(I)$ and the contrast

$$
\begin{aligned}
& V_{I}=\frac{\sigma_{I}}{\langle I\rangle}, \\
& \sigma_{I}^{2}=\left\langle I^{2}\right\rangle-\langle I\rangle^{2},
\end{aligned}
$$

where $\langle I\rangle$ and $\sigma_{I}^{2}$ are the mean intensity and the variance of the intensity fluctuations, respectively. In certain cases, statistical moments of higher orders are employed.
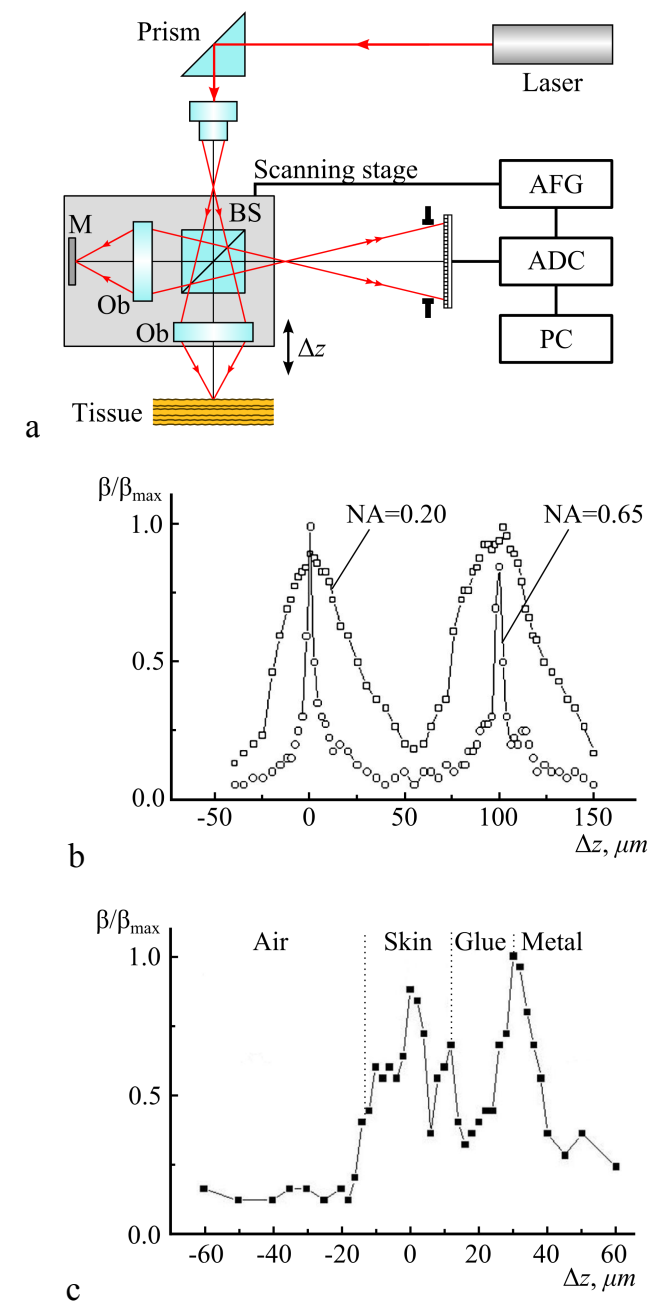

Fig. 3.20 Laser speckle interferometer with the matched wave fronts (a): Laser - He-Ne laser (633 nm), Ob objectives, BS - beam splitter, $\mathrm{M}$ - reference mirror, $\mathrm{PD}$ - photodetector, AFG - audio-frequency generator, ADC - analog-to-digital converter, PC - personal computer; the normalized interferometer signal as a function of depth for a test-object $-150 \mu \mathrm{m}$ planeparallel glass plate $(n \cong 1.5)(\mathrm{b})$; in-depth profiling of the metal-glue-stripped human skin epidermal layer (c) $[5,59]$.

For ideal conditions, when the complex amplitude of scattered light has a Gaussian statistics, the contrast is $V_{I}$ $=1$ (developed speckles), and the intensity probability distribution is represented by a negative exponential function:

$$
p(I)=\frac{1}{\langle I\rangle} \exp \left\{-\frac{I}{\langle I\rangle}\right\} .
$$

Thus, the most probable intensity value in the corresponding speckle pattern is equal to zero, i.e., destructive interference occurs with the highest probability.

Partially developed speckle fields are characterized by a contrast $V_{I}<1$. The contrast may be lower due to a 
uniform coherent or incoherent background added to the speckle field. For the phase objects with the variance of the phase fluctuations $\sigma_{\phi}^{2}>1$ and a small number of scatterers within a focused laser beam contributing to the field at a certain point in the observation plane, the contrast of the speckle pattern is greater than unity. The statistics of the speckle field in this case is nonGaussian and nonuniform (i.e., the statistic parameters depend on the observation angle). The specific features of the diffraction of focused laser beams from moving phase screens underlie speckle methods of structural diagnostics and monitoring of motion parameters of tissues and flows of blood and lymph.

Statistics of the second order shows how fast the intensity changes from point to point in the speckle pattern, i.e., characterizes the size and the distribution of speckle sizes in the pattern. Statistics of the second order is usually described in terms of the autocorrelation function of intensity fluctuations,

$$
G_{2}(\Delta \xi)=\langle I(\xi) \cdot I(\xi+\Delta \xi)\rangle
$$

and its Fourier transform, representing the power spectrum of a random process; $\xi \equiv x$ or $t$ is the spatial or temporal variable; $\Delta \xi$ is the change in variable. Angular brackets \langle\rangle in Eq. (3.65) stand for the averaging over an ensemble or the time.

\subsubsection{Speckle interferometry}

Often regular and random interference interact between each other when interferometric or holography systems are used for study of scattering tissues. Figure 3.20 presents a dual-beam (two-mirror) speckle interferometer, where focused beams are used and as an objective mirror a tissue sample serves [5, 59]. When reflected light forming a developed speckle pattern retains linear polarization, intensity distribution at the output of such interferometer can be written as

$$
\begin{aligned}
& I(r, t)=I_{r}(r)+I_{s}(r)+2\left[I_{r}(r) I_{s}(r)\right]^{1 / 2} \times \\
& \times\left|\gamma_{11}(\Delta t)\right| \cos \left\{\Delta \Phi_{I}(r)+\Delta \Psi_{I}(r)+\Delta \Phi_{I}(r)\right\},
\end{aligned}
$$

where $I_{r}(r)$ and $I_{s}(r)$ are intensity distributions of the reference and signal fields, respectively; $r$ is the transverse spatial coordinate; $\gamma_{11}(\Delta t)$ is the degree of temporal coherence of light; $\Delta \Psi_{I}(r)$ is the deterministic phase difference of the interfering waves; $\Delta \Phi_{I}(r)=\left[\Phi_{I r}(r)-\Phi_{I S}(r)\right]$ is the random phase difference; and $\Delta \Phi_{I}(t)$ is the time-dependent phase difference related to the motion of an object. In particular, for the longitudinal sinusoidal oscillations of one of the interferometer mirrors (reference mirror $M$ or object itself) with the amplitude $l_{0}$ and angular frequency $\Omega_{v}$

$$
\Delta \Phi_{I}(t)=\frac{4 \pi}{\lambda} l_{0} \sin \left(\Omega_{v} t\right)
$$

In the absence of speckle modulation, the deterministic phase difference $\Delta \Psi_{I}(r)$ governs the formation of regular interference fringes as in conventional interferometry. The output signal of a speckle interferometer is maximal for the phasematched interfering fields, when $\Delta \Psi_{I}(r)=$ const within the aperture of the detector, and for interference of focused laser beams, when speckles with maximal sizes are generated. For the large aperture of a photodetector, when speckles are not resolved, the output signal of the interferometer

$$
\beta(\Delta z)=\frac{\sin \left(\frac{\pi(\mathrm{NA})^{2} n \Delta z}{\lambda_{0}}\right)}{\frac{\pi(\mathrm{NA})^{2} n \Delta z}{\lambda_{0}}},
$$

where NA is the numerical aperture of the objective, $\lambda_{0}$ is the wavelength in free space; $n$ is the index of refraction of a sample (tissue), $\Delta z$ is the in-depth displacement. It follows that the in-depth spatial resolution $(\beta \rightarrow 0)$ of the interferometer is $\Delta z_{\min }=\frac{\lambda_{0}}{n(\mathrm{NA})^{2}}$, thus for the objectives with NA close to unity, resolution is close to the wavelength. The normalized interferometer's signal as a function of depth for a test-object $-150 \mu \mathrm{m}$ plane-parallel glass plate with index of refraction $n \cong 1.5$ presented in Fig. $3.20 \mathrm{~b}$ demonstrates an increase of spatial in-depth resolution at using of objectives with the bigger numerical aperture. Two - anterior and posterior interfaces of the plate are well resolved. Similar in depth scanning of the in vivo taken glue-stripped human skin stratum corneum sample, which is attached to a metal plate by a glue layer, also shows rather good spatial resolution of the structure of thin tissue layers (Fig. 3.20c). Important to note, that independent measurement of specimen thickness allows one to evaluate mean index of refraction of sample material. The regularly spatially modulated laser beams are often used for surface profiling, laser anemometry of biological fluids, and cytometry [56, 59]. Typically these methods take advantage of a small spacing of interference fringes $\Lambda_{l}$, 


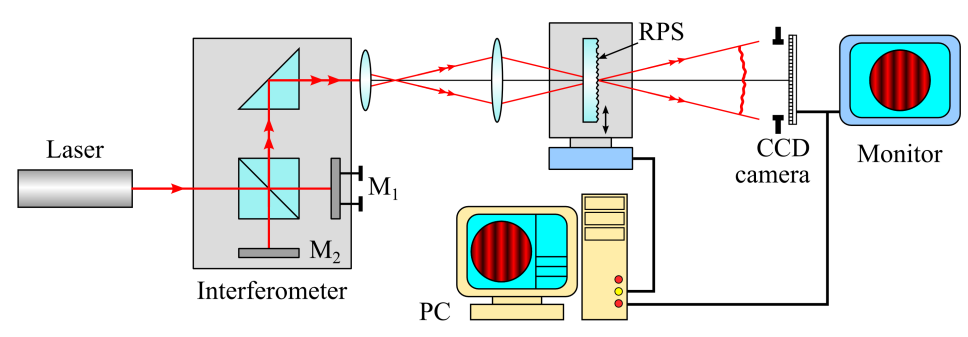

a

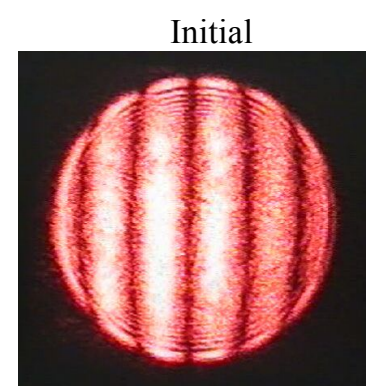

$V=1$

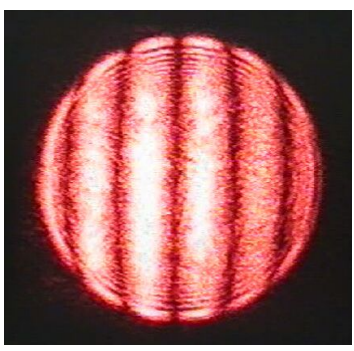

$V=1$

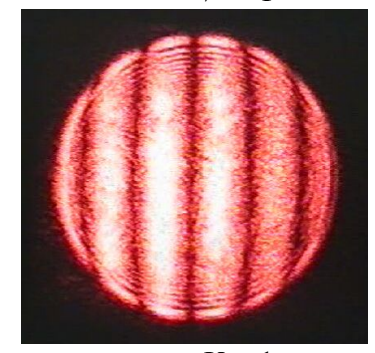

$V=1$

Speckle modulated

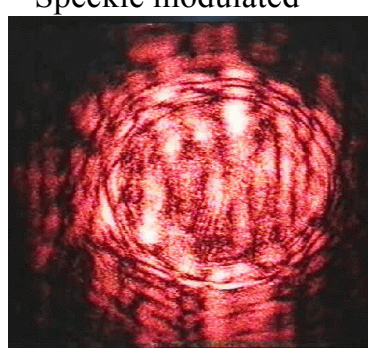

Early senile cataract

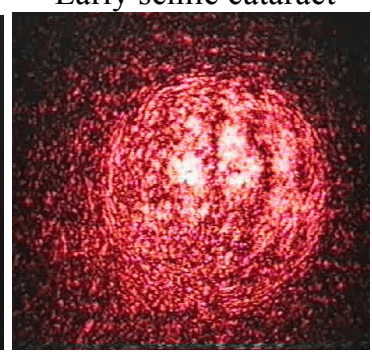

Immature senile cataract

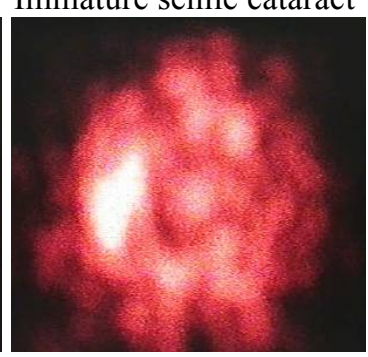

Mature senile cataract

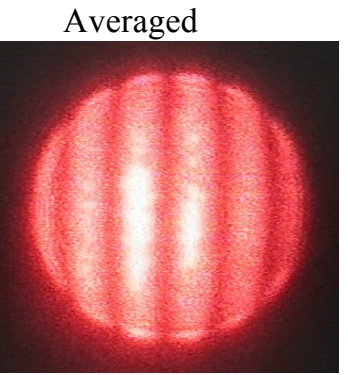

$V=0.25$

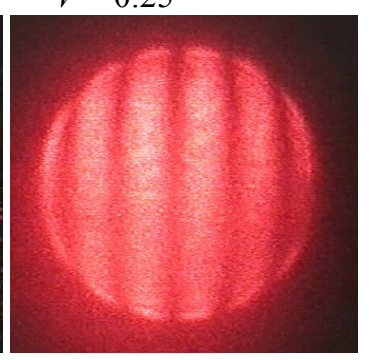

$V=0.23$

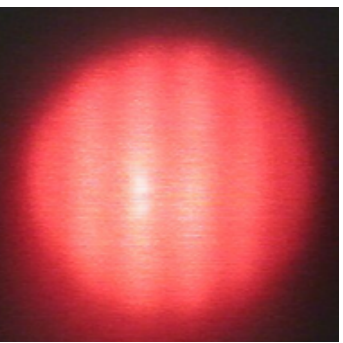

$V=0.15$

b

Fig. 3.21 Interaction of the spatially-modulated laser beam with cataractous eye lenses: experimental setup with external interferometer with the focused beams on the specimen modeled as a random phase screen (RPS) (a); observed interferograms (b): without a specimen (initial, the visibility is equal to unity, $V=1$ ) (left column), with a stationary specimen (cataractous eye lens for different severity of the disease, from early to mature senile cataract)(middle column), and with a movable specimen (averaged) - observation of the average intensity fringes with contrast from $V=$ 0.25 to 0.15 (right column) [5, 59].

$$
\Lambda_{I}=\frac{\lambda}{2 \theta_{I}},
$$

which is comparable to the sizes of inhomogeneities of the object under study. Here $\theta_{I}$ is the angle between the wave vectors of the interfering fields. If the separation between the interference fringes is small, $\Lambda_{I}<d_{a v}$ [the average size of speckles in the observation plane, see Eq. (3.58)], and the diameter of the beam waist $2 w_{0}>$ $L_{\phi}$ [the correlation length of phase fluctuations, see Eq.
(3.61)], the regular interference fringes oriented in a random manner from speckle to speckle are observed within the limits of a single speckle. The contrast of fringes depends in this case only on the relation between the intensities of the interfering fields and does not depend on the statistical properties of an object structure, that gives an opportunity to measure regular phase shifts in the object in spite of its random nature.

The use of modulated beams with a large spacing between interference fringes, which exceed the sizes of inhomogeneities, $\Lambda_{I}>d_{a v}$, results in the appearance of another correlation effects in the scattered field. If the 
beam diameter $2 w_{0}$ and the separation between the fringes $\Lambda_{I}$ are sufficiently large, the fringes modulate the speckle field, and the evolution of the contrast of average-intensity fringes along the beam propagation (along $z$-axis) is determined by the statistical parameters of the object:

$$
\bar{V}_{I}(z)=\left|r_{c}(z)\right| V_{I}
$$

where $V_{I}$ is the contrast in the initial laser beam and $\left|r_{c}(z)\right|$ is the modulus of the transverse correlation coefficient of the complex amplitude of the scattered field. Contrast of the interferential fringes is measured for the incident light as

$$
V_{I}=\frac{I_{\max }-I_{\min }}{I_{\max }+I_{\min }}
$$

and for light propagating after the object at interferential pattern averaging by transverse scanning, $\bar{V}_{I}(z)$ is measured using the similar formula for each $z$ position.

When a spatially modulated light beam is focused using a diffraction-limited optical system with an aperture $D>\Lambda_{I}$, two spatially separated light spots are produced in the area of focusing. The optical scheme for such system is presented in Fig. 3.21a. Since two different areas of an object are irradiated, the interaction of these light spots with a scattering medium gives rise to two completely nonidentical (noncorrelated) speckle fields in the diffraction field. If $\Lambda_{I}>d_{a v}$ and $2 w_{0}>L_{\phi}$, no fringes occur in the scattered field (see Fig. 3.21b). However, when an object moves (scanned) in the transverse direction, a set of average-intensity interference fringes arises (see Fig. 3.21b, right column). The contrast of this pattern is determined by the statistical properties of the specimen structure expressed as the standard deviation $\sigma_{\phi}$ and the correlation length $L_{\phi}$ of phase fluctuations of the interacting field.

\subsection{Dynamic light scattering}

\subsubsection{Quasi-elastic light scattering (QELS) and Doppler effect}

Scattering of light by movable particles is called dynamic light scattering in contrast to scattering of light by none movable or static particles, which is called static or elastic scattering as it occurs without energy transfer $[5,9,43-56,60]$. Instead, at scattering by a movable particle small but measurable light energy change occurs, thus dynamic light scattering is often called as a quasi-elastic light scattering (QELS). The basic phenomenon for QELS is Doppler effect that determines the frequency shift of the scattered light by a well-known expression valid for non-relativistic velocities (Fig. 3.22)

$$
\begin{aligned}
& \Delta \mathrm{v}_{D}=\left(\mathrm{v}_{s}-\mathrm{v}_{0}\right)= \\
& =\mathrm{v}_{0} \frac{\mathrm{v}}{c}\left(\cos \theta_{i}-\cos \theta_{s}\right)=\left(\vec{k}_{s}-\vec{k}_{0}\right) \overline{\mathrm{v}}
\end{aligned}
$$

were $v_{s}$ is the frequency of the scattered wave, $v_{0}$ is the frequency of the incident wave, $v$ is the velocity of the scatterer (particle), $c$ is the light speed, $\theta_{i}$ is the angle between wave vector of the incident light $\vec{k}_{0}$ and direction of particle motion $\vec{v}$, and $\theta_{s}$ is the angle between directions of particle motion $\vec{v}$ and detection of the scattered radiation $\vec{k}_{s}$.

The Doppler shift is proportional to velocity of the light scattering particle. Therefore, measurement of the Doppler shift for known geometry of the experiment (angles $\theta_{i}$ and $\theta_{s}$ ) allows one to determine velocity of a particle or ensemble of the co-directional particles. This is the principle of the Doppler velocimetry. However for living systems different velocities and directions are more characteristic. Thus, a number of Doppler frequencies are measured, i.e., Doppler spectrum, which in principle allows one to reconstruct particle velocity and direction distributions. Such measurement is recognized as Doppler spectroscopy or QELS spectroscopy, or photon-correlation spectroscopy. The last term underlines a fundamental relationship between spectrum and autocorrelation function of intensity fluctuations caused by Doppler signals from many scatterers.

The Doppler shift induced by blood flow and any other flows in the body is too small to be detected directly by a spectrometer. For example, for typical blood flow velocity of $1 \mathrm{~cm} / \mathrm{s}$, the Doppler shift is less than $10 \mathrm{kHz}$. However, application of lasers and optical mixing (heterodyning or self-beating) technique using a photodetector give a possibility to provide easily such measurements. The current of a photodetector is proportional to light intensity. Thus, with allowance for self-beating of the electric components of the scattered field, the temporal intensity autocorrelation function (AF) $G_{2}(\tau)$ can be measured. For the Gaussian random optical fields, when the scattering field phase and amplitude variations are statistically independent of each other, $G_{2}(\tau)$ is related to the first-order AF by the Siegert formula:

$$
g_{2}(\tau)=\frac{G_{2}(\tau)}{\langle I\rangle^{2}}=A\left\{1+\beta_{s b}\left|g_{1}(\tau)\right|^{2}\right\}
$$

Here, $G_{2}(\tau)$ is defined by Eq. (3.65); $\langle I\rangle$ is the ensemble-averaged intensity; $\tau$ is the delay time; $A=$ $\left\langle i^{2}\right\rangle$ is the mean of the square of the photocurrent, or the 
base line of the AF; $\beta_{s b}$ is the parameter of self-beating efficiency, typically $\beta_{s b} \approx 1$; and

$$
g_{1}(\tau)=\frac{\left\langle E(t) E^{*}(t+\tau)\right\rangle}{\left\langle|E(\tau)|^{2}\right\rangle}
$$

is the normalized temporal AF of the optical field, $E(t)$ is the electrical field for the time $t$ and $E *(t+\tau)$ is the complex conjugate of electrical field for $(t+\tau)$.

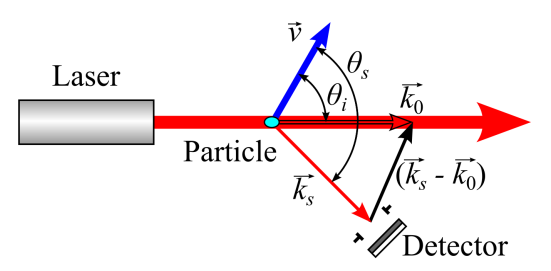

Fig. 3.22 Laser light scattering by a moving particle: $\theta_{i}$ is the angle between wave vector of the incident light $\vec{k}_{0}$ and direction of particle motion $\vec{v}, \theta_{s}$ is the angle between directions of particle motion $\vec{v}$ and detection of the scattered radiation $\vec{k}_{s}[55]$.

For a monodisperse system of Brownian particles

$$
g_{1}(\tau)=\exp \left(-\Gamma_{T} \tau\right)
$$

where

$$
\Gamma_{T}=q^{2} D_{T}
$$

is the relaxation parameter,

$$
q=\left|\vec{k}_{s}-\vec{k}_{0}\right|=\frac{4 \pi}{\lambda} \sin \frac{\theta}{2}
$$

is the module of the scattering wave vector, $\theta=\theta_{s}-\theta_{i}$ (Fig. 3.22);

and

$$
D_{T}=\frac{k_{B} T}{6 \pi \eta_{m} r_{h}}
$$

is the coefficient of translation diffusion, $k_{B}$ is the Boltzmann constant, $T$ is the absolute temperature, $\eta_{m}$ is the absolute viscosity of the medium, and $r_{h}$ is the hydrodynamic radius of a particle.

One of the aims of QELS spectroscopy is the reconstruction of size distribution of scattering particles (biological molecules, cell organelles, cells, etc.) which is the valuable information for early diagnostics and noninvasive monitoring of some diseases. However, QELS algorithms work well only for a single scattering regime.

\subsubsection{Dynamic speckles}

When random moving (dynamic) object is irradiated by a coherent light a dynamic speckle-pattern is formed [5, $9,43-56,60,61]$. This means that phase and intensity of scattered light have a pure stochastic nature. Laser speckle-correlation technique is based on the space-time correlation properties of dynamic speckle field. It allows one to measure the scattering object velocity.

If the scatterers are in random motion, e.g., in Brownian motion, optical speckles obey to the Gaussian statistics and the spatial-temporal correlation function of the complex amplitude of the scattered light can be factorized and presented as a product of spatial and temporal correlation functions. On the other hand, tissue is more appropriate to be modeled as a moving deep random phase screen (RPS) for which the spatial and temporal intensity fluctuations are not statistically independent from each other, thus a spatial-temporal correlation function is introduced to describe dynamic speckles.

For a diffuse object moving in-plane with velocity $\vec{v}$ (Fig. 3.23) and contained a large number of randomly distributed scattering centers within irradiating laser beam with assuming that phase fluctuations of the scattered field obey to the Gaussian statistics and the phase variation is large $\sigma_{\phi}^{2}>>1$, the normalized spatial-temporal correlation function of stationary process may be written as

$$
g_{2}(\vec{r}, \tau)=\frac{\left\langle I_{1} I_{2}\right\rangle}{\left\langle I_{1}\right\rangle\left\langle I_{2}\right\rangle}=1+\left|g_{1}(\vec{r}, \tau)\right|^{2},
$$

where

$$
g_{1}(\vec{r}, \tau)=\frac{\left\langle E_{1} E_{2}^{*}\right\rangle}{\sqrt{\left\langle E_{1} E_{1}^{*}\right\rangle\left\langle E_{2} E_{2}^{*}\right\rangle}}
$$

is the spatial-temporal correlation function of the complex amplitude of the scattered field; $\vec{r}=\vec{X}_{1}-\vec{X}_{2}$, $\tau=t_{1}-t_{2}$.

For the specific case of Gaussian laser beam illumination [see Eq. (1.5)] [1] with the beam axis normal to the object plane and beam waist apart at a distance $z$ from the object plane (see Fig. 3.23), the beam spot radius $w_{b}$ and radius of wavefront curvature $\rho_{b}$ in the object plane are determined by the following expressions [55]: 


$$
w_{b}=w_{0}\left[1+\left(\frac{z}{a_{b}}\right)^{2}\right]^{1 / 2}, \rho_{b}=z\left[1-\left(\frac{a_{b}}{z}\right)^{2}\right],
$$

where $a_{b}=\pi w_{0}^{2} / \lambda$, and the normalized spatialtemporal correlation function of intensity of scattered light in observation plane, which is apart at the distance $l$ from the object plane, is denoted as

$$
\begin{aligned}
& g_{2}(\vec{r}, \tau)-1= \\
& =\exp \left(-\frac{|\vec{v}|^{2}}{w_{b}^{2}} \tau^{2}\right) \exp \left(-\frac{1}{r_{s}^{2}}\left|\vec{r}-\left(1+\frac{l}{\rho_{b}}\right) \vec{v} \tau\right|^{2}\right),
\end{aligned}
$$

where,

$$
r_{s}=\frac{2 l}{k_{0} w_{b}}
$$

is the mean speckle size in the observation plane. As can be seen from Eq. (3.82), speckles translate in the observation plane with velocity differ from the velocity of the object (RPS) $\vec{v}$

$$
\vec{v}_{s}=\frac{\vec{r}}{\tau}=\left(1+\frac{l}{\rho_{b}}\right) \vec{v} .
$$

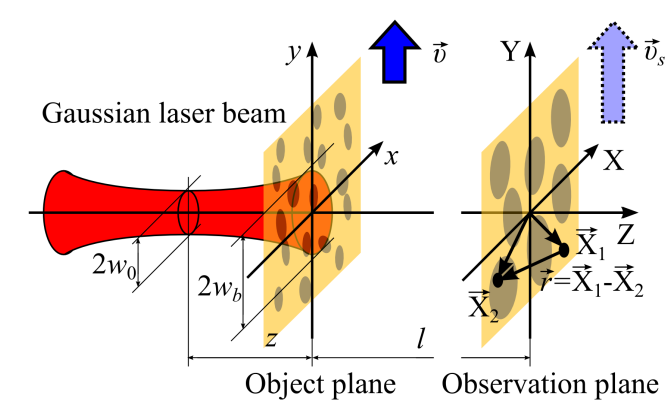

Fig. 3.23 Scattering of the Gaussian laser beam by a random phase screen moving with a velocity $\vec{v}$ in perpendicular direction to the beam axis [55].

The motion of dynamic speckles may arise in two different modes, namely, speckles moving without changing the structure, called "speckle translation" and the speckle changing only the structure in time without translation, called "speckle boiling". The "speckle boiling" mode is characteristic for the random distribution of particle velocities. For the regular velocity of the RPS, speckles also move in a regular manner, thus "speckle translation" mode is realized. For flows of liquids, the combination of these two modes is typical.
In pure translational mode the time, which is required to change the realization of scatterers under the illuminating beam, is equal to

$$
\tau_{T}=\frac{w_{b}}{|\vec{v}|}
$$

Taking into account Eq. (3.84) the translation distance can be estimated as:

$$
r_{T}=\left(1+\frac{l}{\rho_{b}}\right) w_{b} .
$$

The described dependence of speckle dynamics on velocity of the object is the basis of the specklecorrelation technique of velocity measurement. The main idea of this technique is to measure speckle translation velocity in the observation plane and then recalculate the object velocity using Eq. (3.84). One of the simplest way to measure the velocity of speckle translation is to record the intensity fluctuation at two spatially separated points in the observation plane. If distance between observation points is less than $r_{T}$ two similar records delayed by the time $\tau_{d}$ will be obtained. Value $\tau_{d}$ exactly corresponds to the time, which is required for speckle motion from one point to another. It could be easily measured using cross-correlation technique.

\subsubsection{Speckle pattern blurring effect - full-field velocity measurements}

The spatial distribution of contrast of a dynamic speckle pattern at its time-integration can be employed as a detecting parameter to provide a full-field velocity measuring technique [5, 46-55]. If the integration time is comparable with the period of the intensity fluctuations caused by dynamic light scattering, the effect of coherent light interaction with a RPS will be a blurring of the recorded speckle pattern - a reduction in the speckle contrast. It is clear that a very short exposure time would "freeze" the speckle and result in a high-contrast speckle pattern, whereas a long exposure time would allow the speckles to average out, leading to a low contrast.

The use of such time-integrated speckle led in the early 1980 s to a technique for blood flow imaging that simultaneously achieves full-field operation and robust data collection and processing. Originally called "single-exposure speckle photography", it has been developed into a fully-digital, real-time technique for the mapping of capillary blood flow of skin and other tissues and called "laser speckle contrast analysis" (LASCA).

LASCA uses only a laser with diverging optics, a CCD camera, a frame-grabber and a PC. Specially developed software computes the local contrast and converts it to a false-color map of contrast (and hence of velocity). The contrast is quantified by the ratio of the 
standard variation of the intensity fluctuations to the mean intensity, $\frac{\sigma_{I}}{\langle I\rangle}$ [see Eq. (3.62)]. The image is a time-integrated exposure, but for capillary blood flow the exposure is short enough to render the technique effectively real-time. Figure 3.24 illustrates the simplicity of the LASCA basic setup. Light from the laser is diverged by simple optics to illuminate the area under investigation. The CCD camera images the illuminated area, the frame-grabber captures an image and the software immediately processes it to produce a false-color contrast map indicating velocity variations which is displayed by PC monitor. This is typically accomplished in less than one second, making the technique effectively real-time. The number of pixels over which the speckle contrast is computed is of great importance because for a few pixels used the statistics will be questionable, and for too many - the spatial resolution will be lost. In practice, a square of $7 \times 7$ or $5 \times 5$ pixels is usually a satisfactory compromise.

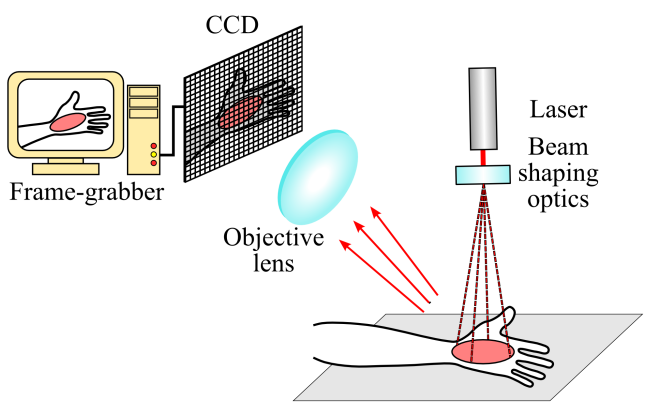

Fig. 3.24 Full-field imaging of dynamic speckles [60].

By making certain assumptions, the following mathematical relationship between the speckle contrast and the temporal statistics of the fluctuating speckles can be found:

$$
\sigma_{I}^{2}(T)=\frac{1}{T} \int_{0}^{T} \tilde{G}_{2}(\tau) \mathrm{d} \tau
$$

where $\sigma_{I}^{2}$ is the spatial variance of the intensity in the speckle pattern; $T$ is the integration time and $\tilde{G}_{2}(\tau)$ is the autocovariance of the temporal intensity fluctuations of a single speckle; $\tilde{G}_{2}(\tau)$ is defined as [see Eq. (3.65)]

$$
\tilde{G}_{2}(\Delta \xi)=[I(\xi)-\langle I\rangle] \cdot[\langle I(\xi+\Delta \xi)-\langle I\rangle\rangle] .
$$

This fundamental equation defines the relationship between LASCA and those techniques that measure the intensity fluctuations directly. LASCA measures the quantity on the left side of Eq. (3.87); photon correlation spectroscopy, laser Doppler, and timevarying speckle techniques measure the quantity on the right side. In addition, LASCA uses image speckles, whereas most of the temporal techniques use far-field speckles. However, this does not detract from the fundamental equivalence of the two approaches expressed in Eq. (3.87).

All the techniques allow for the determination of the correlation time of intensity fluctuations $\tau_{\mathrm{c}}$. The photon correlation technique measures this parameter directly. In the case of LASCA, some further assumptions must be made in order to link the measurement of speckle contrast with $\tau_{\mathrm{c}}$. For example, for the case of a Lorentzian velocity distribution, this relation has a view:

$$
\frac{\sigma_{I}}{\langle I\rangle}=\left[\frac{\tau_{c}}{2 T}\left\{1-\exp \left(-\frac{2 T}{\tau_{c}}\right)\right\}\right]^{1 / 2}
$$

LASCA, as all the temporal measurement techniques, suffers on the problem of relating the correlation time $\tau_{c}$ to the velocity distribution of the scatterers. The relation depends on the multiple scattering, the size and the shape of the scattering particles, non-Newtonian flow, non-Gaussian statistics resulting from a low number of scatterers in the measuring volume, spin of the scatterers, etc. Because of the uncertainties caused by these factors, it is common in all these techniques to rely mainly on calibration rather than on absolute measurements.

\subsubsection{Diffusion wave spectroscopy}

A fundamental difference of diffusion wave spectroscopy (DWS) compared to the QELS spectroscopy is that this approach is applicable in the case of dense media with multiple scattering, which is very important for tissues $[5,52,53,61]$. It is assumed thereby that due to multiple scattering the each photon that has reached given observation point of the detector experiences a great number of scattering events $N$ (Fig. 3.25). The successive scattering acts taking place at the time instant $t$ at the scattering particles located in points $\vec{r}_{1}(t), \vec{r}_{2}(t), \ldots \vec{r}_{i}(t) \ldots \vec{r}_{N}(t)$ of the medium with wave vectors $\vec{k}_{1}, \vec{k}_{2}, \ldots \vec{k}_{i} \ldots \vec{k}_{N}$, result in formation of the field $\vec{E}(t)$, whose total phase change $\Delta \phi(t)$ is determined as:

$$
\Delta \phi(t)=\sum_{i=0}^{N} \vec{k}_{i}(t)\left[\vec{r}_{i+1}(t)-\vec{r}_{i}(t)\right]
$$

$\Delta \phi(t)$ is dependent on the total path length $L$ of each photon migrated from the source $\vec{r}_{0}$ to the detector $\vec{r}_{N+1}$ points (Fig. 3.25):

$$
L=\sum_{i=0}^{N}\left|\vec{r}_{i+1}(t)-\vec{r}_{i}(t)\right|=\sum_{i=0}^{N}\left(\frac{\vec{k}_{i}}{\left|\vec{k}_{i}\right|}\right)\left[\vec{r}_{i+1}(t)-\vec{r}_{i}(t)\right] .
$$


The total mean photon path length $L$ is related to the number of scattering acts $N$ by the relation $L=N l_{s}$, where $l_{s}=\left(\mu_{s}\right)^{-1}$.

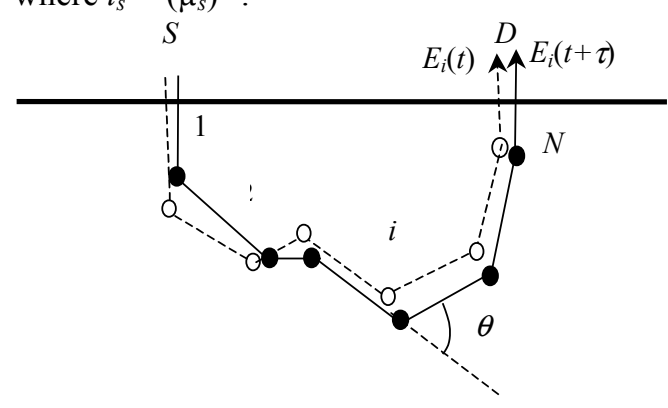

Fig. 3.25 Schematic diagram of the coherent radiation propagation through a randomly inhomogeneous semiinfinite medium with a multiple scattering; light passes from the radiation source $(S)$ towards the detector $(D)$; the location of scattering particles at the time instant $\tau$ is indicated by (o) and at the time instant $(t+\tau)-$ by $(\bullet) ; 1$, $2, \ldots, i, \ldots, N$ are the first-, second-, $i$-th-, and N-th-step of scattering; $\theta$ is the scattering angle.

In contrast to the case of single scattering, the autocorrelation function (AF) of the field $g_{1}(\tau)$ [see Eq. (3.74)] is sensitive to the motion of a particle on the length scale on the order of $\lambda \sqrt{l_{t r} / L}$, which is generally much less than $\lambda$, because $L>l_{t r}$ [see Eqs. (3.42) and (3.43)]. Thus, DWS AFs decay much faster than AFs employed in QELS.

Experimental implementation of DWS is very simple. A measuring system should provide irradiation of an object under study by a CW laser beam and measurement of intensity fluctuations of the scattered radiation within a single speckle with the use of a single-mode receiving fiber, photomultiplier, photoncounting system, and a fast digital correlator working in a nanosecond range. The possibilities of the DWS technique for medical applications have been demonstrated for the blood flow monitoring in the human forearm and brain. The AF slope is the indicative parameter for determination of the blood flow velocity. The normalized AF of field fluctuations can be represented in terms of two components related to the Brownian and directed motion of scatterers (erythrocytes):

$$
g_{1}(\tau) \approx \exp \left\{-2\left[\frac{\tau}{\tau_{B}}+\left(\frac{\tau}{\tau_{S}}\right)^{2}\right] \frac{L}{l_{t r}}\right\},
$$

where $\quad \tau_{B}^{-1} \equiv \Gamma_{T}=q^{2} D_{T} \quad$ [see $\quad$ Eq. (3.76)], $\tau_{S}^{-1} \cong 0.18 G_{V} q l_{t r}$ characterizes the directed flow, and $G_{V}$ is the gradient of the flow rate. It allows one to express the slope of the AF in terms of the diffusion coefficient $D_{T}$ (characterizes blood microcirculation) and the gradient of the directed velocity of blood $G_{V}$.
When spatially-resolved single speckle detection is provided, the technique is transformed to diffusion wave imaging (DWI) mode.

\subsection{Interaction of the polarized light with tissues}

\subsubsection{Definitions}

Light of arbitrary polarization can be represented by four numbers known as the Stokes parameters, $I, Q, U$, and $V: I$ - refers to the intensity of the light; the parameters $Q, U$, and $V$ represent the extent of horizontal liner, $45^{\circ}$ linear, and circular polarization, respectively $[5,9,56,62-71]$. In terms of the electric field components the Stokes parameters are given by

$$
\begin{aligned}
& I=\left\langle E_{\|} E_{\|}^{*}+E_{\perp} E_{\perp}^{*}\right\rangle, \\
& Q=\left\langle E_{\|} E_{\|}^{*}-E_{\perp} E_{\perp}^{*}\right\rangle, \\
& U=\left\langle E_{\|} E_{\perp}^{*}+E_{\perp} E_{\|}^{*}\right\rangle, \\
& V=\left\langle i\left(E_{\|} E_{\perp}^{*}-E_{\perp} E_{\|}^{*}\right)\right\rangle,
\end{aligned}
$$

and the irradiance or intensity of light by

$$
I^{2} \geq Q^{2}+U^{2}+V^{2}
$$

For an elementary monochromatic plane or spherical electromagnetic wave, Eq. (3.94) is the equality. For a partially polarized quasi-monochromatic light which can be presented as a mixture of elementary waves, the Stokes parameters are sums of the respective Stokes parameters of these elementary waves, because of fundamental property of additivity. In this case Eq. (3.94) is the inequality.

The Stokes vector $\vec{S}$ of a light beam is constructed basing on six flux measurements obtained with different polarization analyzers in front of the detector:

$$
\vec{S}=\left(\begin{array}{c}
I \\
Q \\
U \\
V
\end{array}\right)=\left(\begin{array}{c}
I_{H}+I_{V} \\
I_{H}-I_{V} \\
I_{+45^{\circ}}-I_{-45^{\circ}} \\
I_{R}-I_{L}
\end{array}\right)
$$

where $I_{H}, I_{V}, I_{+45}, I_{-45^{\circ}}, I_{R}$, and $I_{L}$ are the light intensities measured with a horizontal linear polarizer, a vertical linear polarizer, $\mathrm{a}+45^{\circ}$ linear polarizer, a $-45^{\circ}$ linear polarizer, a right circular analyzer, and a left circular analyzer in front of the detector, respectively. Because of the relationship $I_{H}+I_{V}=I_{+45^{\circ}}+I_{-45^{\circ}}=I_{R}+I_{L}=I$, where $I$ is the intensity of the light beam measured without any analyzer in front of the detector, a Stokes vector can be determined by four independent measurements. 
The degree of linear $(L)$ and circular $(C)$ polarization of the scattered light is defined as

$$
P_{L}=\frac{I_{\|}-I_{\perp}}{I_{\|}+I_{\perp}}=\frac{\sqrt{Q_{s}^{2}+U_{s}^{2}}}{I_{s}},
$$

and

$$
P_{C}=\frac{\sqrt{V_{s}^{2}}}{I_{s}}
$$

The polarization state of the scattered light in the far zone is described by the Stokes vector connected with the Stokes vector of the incident light

$$
\vec{S}_{S}=\mathbf{M} \times \vec{S}_{i}
$$

where $\boldsymbol{M}$ is the normalized $4 \times 4$ scattering matrix (intensity or Mueller matrix)

$$
\mathbf{M}=\left[\begin{array}{llll}
M_{11} & M_{12} & M_{13} & M_{14} \\
M_{21} & M_{22} & M_{23} & M_{24} \\
M_{31} & M_{32} & M_{33} & M_{34} \\
M_{41} & M_{42} & M_{43} & M_{44}
\end{array}\right],
$$

and $\vec{S}_{i}$ is the Stokes vector of the incident light.

The light scattering matrix (LSM) elements depend on the scattering angle $\theta$, the wavelength, and geometrical and optical parameters of the scatterers. There are only seven independent elements (of sixteen) for the scattering matrix of a single particle with fixed orientation and nine relations, which connect the others together. For scattering by a collection of randomly oriented scatterers, there are 10 independent parameters.

The element $M_{11}$ is what is measured when the incident light is unpolarized. The angular dependence of $M_{11}(\theta)$ is the phase function of the scattered light for the single scattering mode [see Eqs. (3.27), (3.37), and (3.38)]. The $M_{11}$ is much less sensitive to chirality and long-range structure than some of the other matrix elements. The $M_{12}$ element refers to a degree of linear polarization of the scattered light. A good measure of scatterer nonsphericity is the $M_{22}$ element which displays the ratio of depolarized light to the total scattered light. The unique characteristic for different biological systems is the $M_{34}$ element which displays the transformation of $45^{\circ}$ obliquely linear polarized incident light to circularly polarized scattered light, because many tissues or tissues constitutes are birefringent and work as a phase plate (retarder). The difference between the $M_{33}$ and $M_{44}$ elements is also a good measure of scatterer nonsphericity.
In addition to definitions of degree of polarization [Eqs. (3.96) and (3.97)], the diattenuation (linear dichroism) is introduced as

$$
D_{A}=\frac{P_{1}^{2}-P_{2}^{2}}{P_{1}^{2}+P_{2}^{2}}=\frac{\sqrt{M_{12}^{2}+M_{13}^{2}+M_{14}^{2}}}{M_{11}},
$$

where $P_{1}$ and $P_{2}$ are the coefficients characterizing the amplitude transmission for the two orthogonal linear polarization states.

To describe polarization-sensitive interaction of light with tissues, different optical models are applicable. For the small particle systems, scattering can be described using Rayleigh theory (see subsection 3.2.3, Eq. (3.23) and Fig. 3.10). For a special class of arbitrary shaped particle systems, where $|m-1|<<1$ and $\frac{2 \pi a^{\prime}}{\lambda}|m-1|<<1$, $a^{\prime}$ is the largest dimension of the particle, the RayleighGans or Rayleigh-Debye theory can be used for calculating the scattering properties. For tissues in the NIR $a^{\prime}$ could be up to $850-950 \mathrm{~nm}$. For describing of near the forward direction scattering caused by large particles (of order of $10 \mu \mathrm{m}$ ) the Fraunhofer diffraction approximation is useful. According to this theory, the scattered light has the same polarization as that of the incident light and the scatter pattern is independent of the refractive index of the object. For the small scattering angles, Fraunhofer diffraction approximation can represent accurately the change in irradiance as a function of particle size. That is why this approach is applicable in the laser flow cytometry. The structure of the biological cell such as cell membrane, nuclear texture, and granules in the cytoplasm can be represented by variations in optical density.

Mie or Lorenz-Mie scattering theory is an exact solution of Maxwell's electromagnetic field equations for a homogeneous sphere (see subsection 3.2.4). In the general case, light scattered by a particle becomes elliptically polarized. For spherically symmetric particles of an optically inactive material the Mueller scattering matrix is given by

$$
\begin{aligned}
& \mathbf{M}(\theta)= \\
& =\left[\begin{array}{cccc}
M_{11}(\theta) & M_{12}(\theta) & 0 & 0 \\
M_{12}(\theta) & M_{22}(\theta) & 0 & 0 \\
0 & 0 & M_{33}(\theta) & M_{34}(\theta) \\
0 & 0 & -M_{34}(\theta) & M_{44}(\theta)
\end{array}\right] .
\end{aligned}
$$

Mie theory has been extended to arbitrary coated spheres and to arbitrary cylinders. In the Mie theory the electromagnetic fields of the incident, internal and scattered waves are each expanded in a series. A linear transformation can be made between the fields in each 


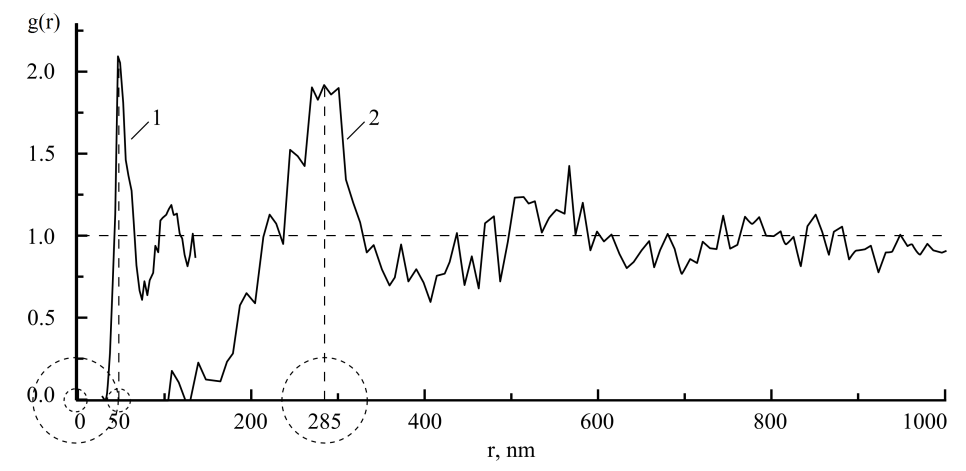

Fig. 3.26 Experimental radial distribution function $g(r)$ for rabbit cornea (1) and human sclera (2); $g(r)$ is proportional to the probability of particle displacement $r$ at a certain distance from an arbitrarily fixed particle (see collagen cylinder faces for rabbit cornea of diameter $\sim 28 \mathrm{~nm}$ and for human sclera of diameter $\sim 100 \mathrm{~nm}$ for $r=0$; the nearest the most probable particle position $r \approx 50 \mathrm{~nm}$ for rabbit cornea and $r \approx 285 \mathrm{~nm}$ for human sclera) [5].

of the regions. This approach can also be used for nonspherical objects such as spheroids. The linear transformation is called the transition matrix (T-matrix). T-matrix for spherical particles is diagonal.

\subsubsection{Single scattering and quasi-ordered tissues}

Many tissues are featured as densely packed disperse systems. Evidently, the interaction of the polarized light with such particle systems may have a number of important peculiarities caused by a specificity of constructive and destructive interference of the scattered waves. For example, eye tissues, such as cornea and lens, in spite of particle structure and refractive index mismatch which causes light scattering by an individual particle, are highly transparent tissues the origin of which is based on their ordered structure (see subsections 2.2 and 2.4, Fig. 2.7) [1]. Light scattering in such systems can be analyzed using the radial distribution function $g(r)$, which statistically describes the spatial arrangement of particles in the system (Fig. 3.26) [5]. For a fibrillar particle system the function $g(r)$ is the ratio of the local number density of the fibril centers at a distance $r$ from a reference fibril at $r=0$ to the bulk number density of fibril centers. It expresses the relative probability of finding two fibril centers separated by a distance $r$; thus, $g(r)$ must vanish for values of $r \leq 2 a$ ( $a$ is the radius of a fibril, because fibrils cannot approach each other closer than touching). The radial distribution function of scattering centers $g(r)$ for a certain tissue may be calculated on the basis of tissue electron micrographs (see Figs. 2.7 [1] and 3.26).

Figure 3.26 depicts a typical result for one of the rabbit cornea regions. The function $g(r)=0$ for $r \leq 25$ $\mathrm{nm}$, which is consistent with a fibril radius of $14 \pm 2 \mathrm{~nm}$, can be calculated from the electron micrograph similar to that shown for the human cornea in Fig. 2.7b [1]. The first peak in the distribution gives the most probable separation distance, which is approximately $50 \mathrm{~nm}$. The value of $g(r)$ is essentially unity for $r \geq 170 \mathrm{~nm}$, indicating that the fibril positions are correlated over no more than a few of their nearest neighbors. Therefore, a short-range order exists in the system.
Similar calculations for several regions of the human eye sclera are also shown in Fig. 3.26. Electron micrographs, such as presented in Fig. 2.7d [1], were processed by averaging for 100 fibril centers. The obtained results present evidence of the presence of a short-range order in the sclera, although the degree of order is less pronounced than in the cornea. The function $g(r)=0$ for $r \leq 100 \mathrm{~nm}$, which is consistent with the mean fibril diameter of $\approx 100 \mathrm{~nm}$ derived from the electron micrograph. The first peak in the distribution gives the most probable separation distance, which is approximately $285 \mathrm{~nm}$. The value of $g(r)$ is essentially unity for $r \geq 750 \mathrm{~nm}$, indicating a short-range order in the system.

For an isotropic system of $N$ identical interacting long cylinders the scattered intensity in a single scattering approximation is defined as $[5,9]$

$$
\langle I\rangle=\left|E_{0}\right|^{2} N S_{2}(\theta)
$$

where $E_{0}$ is the scattering amplitude of an isolated (noninteracting) particle,

$$
\begin{aligned}
& S_{2}(\theta)= \\
& =\left\{1+8 \pi a^{2} \rho_{c} \int_{0}^{R}[g(r)-1] J_{0}\left(\frac{2 \pi a}{\lambda} r \sin \frac{\theta}{2}\right) \mathrm{d} r\right\}
\end{aligned}
$$

is the structure factor; $a$ is the radius of a cylinder face; $\rho_{c}$ is the mean density of cylinder faces; $J_{0}$ is the zero order Bessel function; $R$ is the distance for that $g(r) \rightarrow 1$; $\theta$ is the scattering angle. For an isotropic system of identical spherical particles in a single scattering approximation

$$
\langle I\rangle=\left|E_{0}\right|^{2} N S_{3}(\theta)
$$




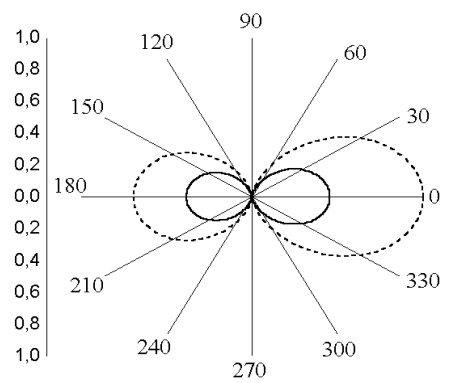

a

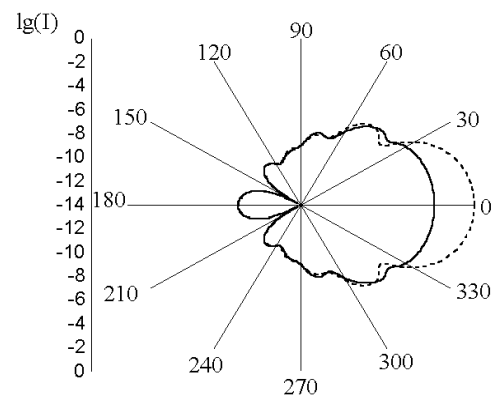

$\mathrm{c}$

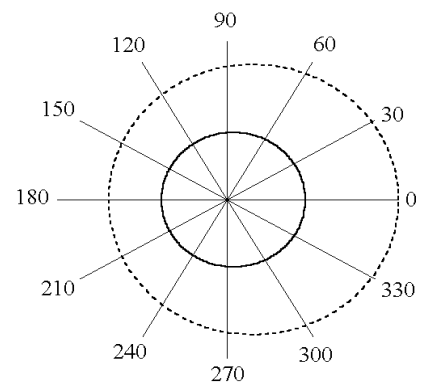

b

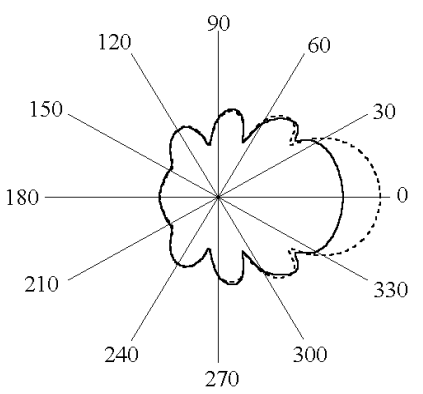

d

Fig. 3.27 The calculated angular dependences of the scattered intensity for systems of small spherical particles $(a=20$ $\mathrm{nm})[(\mathrm{a})$ and (b)] with volume fraction, $f=0.1$, and large spherical particles $(a=500 \mathrm{~nm})[(\mathrm{c})$ and (d)] with volume fraction, $f=0.4$; the incident wave is linearly polarized parallel [(a) and (c)] with or perpendicular [(b) and (d)] to the scattering plane (see Fig. 3.9); dotted line - independent particles; wavelength, $633 \mathrm{~nm}$; relative refraction index, $m=1.105$ (calculated by I.L. Maksimova) [5].

$$
S_{3}(\theta)=\left\{1+4 \pi \rho_{3} \int_{0}^{R} r^{2}[g(r)-1] \frac{\sin q r}{q r} \mathrm{~d} r\right\},
$$

where $q=\frac{4 \pi}{\lambda} \sin \frac{\theta}{2}, \rho_{3}$ is the mean density of particles, and $R$ is the distance for which $g(r) \rightarrow 1$. Quantity $S_{3}(\theta)$ is the $3 \mathrm{D}$ structure factor. This factor describes the alteration of the angular dependence of the scattered intensity that appears with a higher particle concentration.

This model can be used to describe polarized light interaction with anisotropic media showing a single scattering. Many tissues composed from optically soft particles (refractive index mismatch of scatterers and surrounding medium is small) and thin enough, such as cornea, eye lens, mucosa, epithelial layers, can be approximated as single scattering systems. The interference between elementary scattered fields, which is accounted for by structure functions $S_{2}(\theta)$ and $S_{3}(\theta)$, transforms scattering angular dependences of Mueller matrix elements and corresponding optical properties.

Figure 3.27 illustrates the angular dependences of the scattered intensity for systems of small spherical particles $(a=20 \mathrm{~nm})$ with volume fraction, $f=0.1$, and large spherical particles $(a=500 \mathrm{~nm})$ with volume fraction, $f=0.4$. The incident wave is linearly polarized parallel with or perpendicular to the scattering plane (see Fig. 3.9). The dotted lines show angular light distributions for the independent (randomly distributed) particles and solid lines - for the ordered particles, for which interference of the scattered fields play a significant role. The overall scattering is suppressed, for the small particle system such suppression is quite isotropic for both polarization states, and for the large particle system the forward directed scattering is mostly suppressed also for both polarization states. As a result both particle systems became more transparent for the incident light. Important to note that for big particle system results for the scattered intensity are presented in the logarithmic scale.

\subsubsection{Multiple scattering}

Polarized light interactions at light propagation through multiply scattering tissues are fully described by the vector radiative transfer equation (VRTE). For macroscopically isotropic and symmetric plane-parallel scattering media, VRTE can be substantially simplified as follows [5, 29]:

$$
\begin{aligned}
& \frac{\mathrm{d} \vec{S}(\vec{r}, \vartheta, \varphi)}{\mathrm{d} \tau(\bar{r})}=-\vec{S}(\vec{r}, \vartheta, \varphi)+\frac{\Lambda(\vec{r})}{4 \pi} \times \\
& \times \int_{-1}^{+1} \mathrm{~d}\left(\cos \vartheta^{\prime}\right) \int_{0}^{2 \pi} \mathrm{d} \varphi^{\prime} \overrightarrow{\mathbf{Z}}\left(\vec{r}, \vartheta, \vartheta^{\prime}, \varphi-\varphi^{\prime}\right) \vec{S}\left(\vec{r}, \vartheta^{\prime}, \varphi^{\prime}\right)
\end{aligned}
$$




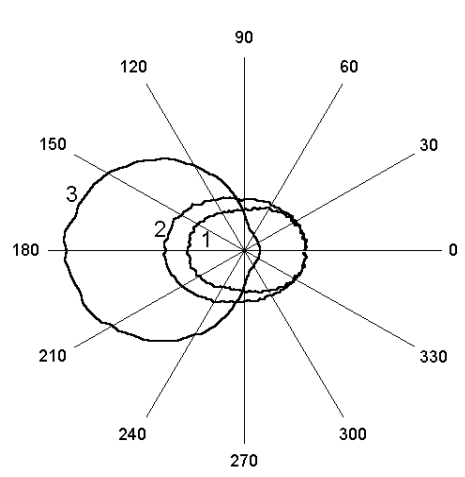

a

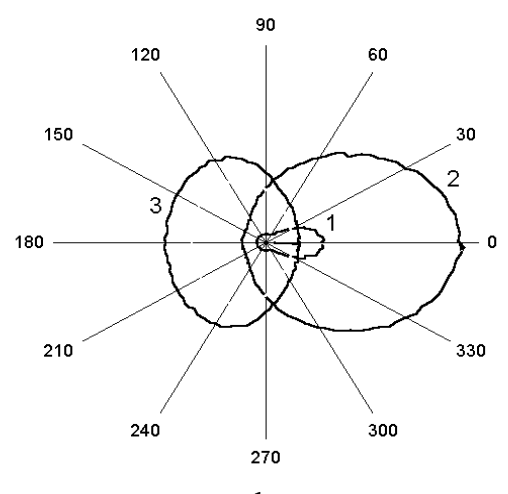

d

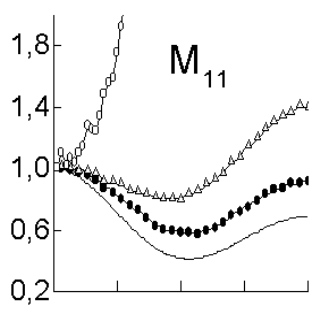

b

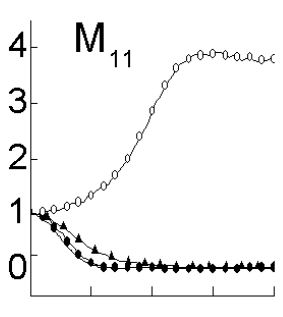

e

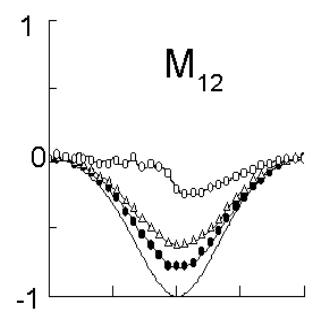

c

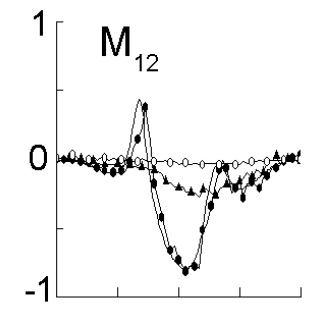

f

Fig. 3.28 The Monte Carlo simulation of the angular scattering and polarization properties for systems of small spherical particles $(a=50 \mathrm{~nm})[(\mathrm{a})-(\mathrm{c})]$ and large spherical particles $(a=300 \mathrm{~nm})[(\mathrm{d})-(\mathrm{f})] ; M_{11}-$ refers to the total scattering intensity and $M_{12}$ - refers to a degree of linear polarization of the scattered light; $\lambda=633 \mathrm{~nm}, m=1.2, f=$ 0.01; single scattering (-); multiple scattering: for the small particles diameter of the spherical system filled up by the particles $-1 \mathrm{~mm}(\bullet), 2 \mathrm{~mm}(\Delta)$, and $20 \mathrm{~mm}(\mathrm{o})$; and for the large particles diameter of the system $-0.002 \mathrm{~mm}(\bullet), 0.2$ $\mathrm{mm}(\Delta)$, and $2 \mathrm{~mm}$ (o) (calculated by I.L. Maksimova) [5].

where $\vec{S}$ is the Stokes vector [see Eq. (3.95)]; $\vec{r}$ is the position vector; $\vartheta, \varphi$ are the angles characterizing incident direction, respectively, the polar (zenith) and the azimuth angles; $\mathrm{d} \tau(\vec{r})=\rho_{p}(\vec{r})\left\langle\sigma_{\text {ext }}(\vec{r})\right\rangle \mathrm{d} s$ is the optical path length element, $\rho_{p}$ is the local particle number density, $\left\langle\sigma_{e x t}\right\rangle$ is the local ensemble-averaged extinction cross section $\left[\sigma_{e x t}=\sigma_{a b s}+\sigma_{s c a}\right.$; see Eqs. (3.13) and (3.17)], $\mathrm{d} s$ is the path length element measured along the unit vector of the direction of light propagation; $\Lambda$ is the single scattering albedo [see Eq. (3.20)]; $\vartheta^{\prime}, \varphi^{\prime}$ are the angels characterizing scattering direction, respectively the polar (zenith) and the azimuth angels; $\overrightarrow{\mathbf{Z}}$ is the normalized phase matrix $\overrightarrow{\mathbf{Z}}\left(\vec{r}, \vartheta, \vartheta^{\prime}, \varphi-\varphi^{\prime}\right)=\mathbf{R}(\Phi) \mathbf{M}(\theta) \mathbf{R}(\Psi)$, where $\mathbf{M}(\theta)$ is the single scattering Mueller matrix [see Eq. (3.99)]; $\theta$ is the scattering angle, and $\mathbf{R}(\phi)$ is the Stokes rotation matrix for angle $\phi$ :

$$
\mathbf{R}(\phi)=\left[\begin{array}{cccc}
1 & 0 & 0 & 0 \\
0 & \cos 2 \phi & -\sin 2 \phi & 0 \\
0 & \sin 2 \phi & \cos 2 \phi & 0 \\
0 & 0 & 0 & 1
\end{array}\right] .
$$

Every Stokes vector and Mueller matrix are associated with a specific reference plane and coordinates. The first term on the right-hand side of VRTE [Eq. (3.106)] describes the change in the specific intensity vector over the distance $d s$ caused by extinction and dichroism (deattenuation), the second term describes the contribution of light illuminating a small volume element centered at $\bar{r}$ from all incident directions and scattered into the chosen direction.

For real systems, the form of VRTE tends to be rather complex and often intractable. Therefore, a wide range of analytical and numerical techniques have been developed to solve the VRTE. Because of important property of the normalized phase matrix, Eq. (3.107), being dependent on the difference of the azimuthal angles of the scattering and incident directions rather than on their specific values, an efficient analytical treatment of the azimuthal dependence of the multiply scattered light, using a Fourier decomposition of the VRTE, is possible. The following techniques and their combinations can be used to solve VRTE: transfer matrix method, the singular eigenfunction method, the perturbation method, the small-angle approximation, the adding-doubling method, the matrix operator method, the invariant embedding method, and the Monte Carlo method. 
When the medium is illuminated by unpolarized light and/or only the intensity of multiply scattered light needs to be computed, the VRTE can be replaced by its approximate scalar counterpart. In that case, in Eq. (3.106), the Stokes vector is replaced by its first element (i.e., radiance) [see Eq. (3.95)] and the normalized phase matrix by its $(1,1)$ element [i.e., the phase function, $p\left(\vec{s}, \vec{s}^{\prime}\right)$ which for scattering symmetric relative to the direction of the incident wave is described by Eqs. (3.21), (3.27), (3.37) or (3.38)].

The results of Monte Carlo simulations for polarized light propagation within the multiple scattering media with parameters close to that of tissues are shown in Fig. 3.28 [5]. These calculations well demonstrate that polarization properties of tissues could be dramatically transformed for the multiple scattering conditions. For systems of small spherical particles, the scattering matrix element $M_{11}$, which refers to the total scattering intensity, shows a significant scattered light redistribution from the mode of isotropic scattering characteristic to single scattering to more intensive scattering mostly in the backward direction as the multiplicity of scattering going up with the increase of diameter of the spherical system filled up by the particles from 1 to $20 \mathrm{~mm}$. The polarization ability of the system originated by Rayleigh (single dipole) scattering (see Fig. 3.27a,b) and expressed in terms of the element $M_{12}$, which refers to a degree of linear polarization of the scattered light, goes down at scattering multiplicity increase. The $100 \%$-polarization efficiency at the scattering angle $\theta=90^{\circ}$ at single scattering mode is reduced significantly, to $\sim 20 \%$. For a large particle system, its transfer from the single to the multiple scattering mode by changing diameter of the system from 0.002 to $2 \mathrm{~mm}$ change angular dependences of the elements $M_{11}$ and $M_{12}$ more dramatically than for small particles. As multiplicity of scattering increase a strong forward scattering transfers to a strong backward scattering with the shape more or less similar to the scattering by small particles, however the polarization ability at $\theta=90^{\circ}$ decreased from $\sim 80 \%$ to a few percents only.

The Mueller matrix for the backscattering geometry was obtained by solving a radiative transfer equation with appropriate boundary conditions. Analysis of this matrix structure showed that its form coincides with the single scattering matrix for optically active spherical scatteres. Thus, different tissues or tissues in pathological or normal functional states should display different responses to the probing with linearly and circularly polarized light. This effect can be employed both in optical medical tomography and for determining optical and spectroscopic parameters of tissues.

The depolarization length in tissues should be close to the mean transport path length $l_{t r}$ of a photon [see Eq. (3.43)], because this length characterizes the distance within which the direction of light propagation and, consequently, the polarization plane of linearly polarized light become totally random after many sequential scattering events (see Fig.3.14).
Since the length $l_{t r}$ is determined by the parameter $g$ characterizing the anisotropy of scattering, the depolarization length should also substantially depend on this parameter. Whole blood is characterized by a considerable depolarization length (around $4 \mathrm{~mm}$ ) at $\lambda=$ $633 \mathrm{~nm}$, which is indicative of the dependence on the parameter $g$, whose value for blood exceeds the values of this parameter for tissues of many other types and can be estimated as $0.982-0.999$.

In contrast to depolarization, the attenuation of collimated light is determined by the total attenuation coefficient $\mu_{t}$ [see Eq. (3.18)]. For many tissues, $\mu_{t}$ is much greater than $\left(\mu_{a}+\mu_{s}{ }^{\prime}\right)$. Therefore, in certain situations, it is impossible to detect pure ballistic photons (photons that do not experience scattering), but forward scattered photons retain their initial polarization and can be used for imaging purpose [5, 9, 10, 71-77]. There was experimentally demonstrated that laser radiation retains linear polarization on the level of $P_{L} \leq$ 0.1 within $2.5 l_{t r}$. Specifically, for skin irradiated in the red and NIR ranges, $\mu_{a} \cong 0.4 \mathrm{~cm}^{-1}, \mu_{s}{ }^{\prime} \cong 20 \mathrm{~cm}^{-1}$, and $l_{t r}$ $\cong 0.48 \mathrm{~mm}$. Consequently, light propagating in skin can retain linear polarization within the length of about 1.2 $\mathrm{mm}$. Such an optical path in a tissue corresponds to a delay time on the order of $5.3 \mathrm{ps}$, which provides an opportunity to produce polarization images of macroinhomogeneities in a tissue with a spatial resolution equivalent to the spatial resolution that can be achieved with the selection of photons by means of more sophisticated time-resolved techniques. In addition, polarization imaging makes it possible to eliminate specular reflection from the surface of a tissue which allows one to apply this technique for the imaging of microvessels in facile skin. Polarization images can see skin subsurface textural changes and allows one to erase melanin from image.

The polarization imaging is a prospective direction in tissue optics $[5,9,10,71-77]$. The registration of two-dimensional polarization patterns for the backscattering of a polarized incident narrow laser beam is the basis for this technique. The major informative images can be received using the backscattering Mueller matrix approach. To determine each of the 16 experimental matrix elements, a total of 16 images should be taken at various combinations of input and output polarization states. Spatially-resolved reflectance and optical coherence tomography (OCT) imaging techniques are well combined with polarization method.

\subsection{Refractive index and controlling of light interaction with tissues}

Index of refraction of tissue compounds is of great importance for light tissue interaction [5, 9]. Most of tissues have refractive indices for visible light in the range of 1.335-1.620 (e.g. 1.55 for the stratum corneum, 1.620 for the enamel, and 1.386 at the lens surface). In the simplest model, the mean refractive index $\bar{n}$ of a tissue can be presented as the weighted 
sum of refractive indices of the scattering center material $n_{s}$ and the ground matter $n_{0}$ :

$$
\bar{n}=f_{s} n_{s}+\left(1-f_{s}\right) n_{0}
$$

where $f_{s}$ is the volume fraction of the scatterers.

The ratio $n_{s} / n_{0} \equiv m$ determines the scattering coefficient. For example, in a simple monodisperse model of scattering dielectric spheres reduced scattering coefficient $\mu_{s}{ }^{\prime}$ is defined by Eq. (3.44). It follows from Eq. (3.44) that even a 5\% change in the refractive index of the ground matter $\left(n_{0}=1.35 \rightarrow 1.42\right)$, when that of the scattering centers is $n_{s}=1.47$, will cause a 7 -fold decrease of $\mu_{\mathrm{s}}^{\prime}$. Therefore, matching of refractive index of the scatterers and ground material allows for considerable reduction of tissue scattering. This phenomenon is very useful for improvement of facilities of optical tomography and for getting more precise spectroscopic information from the depth of a tissue [5, 9, 78-91].

Optical parameters of a tissue, in particular refractive index, are known to depend on water content. In the visible and NIR wavelength range the following formula can be used to evaluate water index of refraction as a major tissue component $(\lambda$ in $\mathrm{nm})$ [5]:

$$
\begin{aligned}
& n_{\mathrm{H}_{2} \mathrm{O}}= \\
& =1.3199+\frac{6878}{\lambda^{2}}-\frac{1.132 \times 10^{9}}{\lambda^{4}}+\frac{1.11 \times 10^{14}}{\lambda^{6}} .
\end{aligned}
$$

For different constituents of a biological cell indices of refraction in the NIR can be evaluated as: extracellular fluid $-\bar{n}=1.35-1.36$, cytoplasm -1.360 -1.375 , cell membrane -1.46 , nucleus $-1.38-1.41$, mitochondria and other organelles - $1.38-1.41$, melanin granules $-1.6-1.7$. Scattering arises from mismatch in refractive index of the components that make up the cell. For tissues when cells are surrounded by other cells or tissue structures of similar index certain organelles become the important scatterers. For instance, the nucleus is significant scatterer because it is often the largest organelle in the cell and its size increases relative to the rest of the cell throughout neoplastic progression. Mitochondria (500-1500 nm in diameter), lysosomes $(500 \mathrm{~nm})$, and peroxisomes (500 $\mathrm{nm}$ ) are very important scatterers whose size relative to the wavelength of light suggests that they must give a significant include in the backscattering. Melanin granule, traditionally thought of as an absorber, must be considered an important scatterer because of its size and high refractive index. Structures consisting of membrane layers such as the endoplasmic reticulum or Golgi apparatus may prove significant because they contain index fluctuations of high spatial frequency and amplitude. Besides cell components, tissue fibrous structures, such as collagen and elastin fibers, must be considered as important scatterers.
Index of refraction of a number of tissues at $633 \mathrm{~nm}$ is in the range from the lowest 1.368 for liver to the largest 1.455 for fatty tissue with other tissues between, such as 1.380 for lungs, 1.400 for blood and spleen, 1.410 for muscular tissue, and 1.418 for kidney. There is a tendency to refraction decrease with the wavelength from 390 to $700 \mathrm{~nm}$, in particular, for bovine muscle from 1.42 to 1.39 .

In terahertz range, $0.5-2.5 \mathrm{THz}$, mean index of refraction of water, the main component of soft tissues, is equal to: $n_{W} \cong 2.2[92,93]$. For the soft tissues which are well supplied by water, such as muscle and skin (dermis), $n \cong 2.1$, for more dry epidermis $n \cong 2.0$; for a soft tissue with a less water content, such as adipose, $n$ $\cong 1.65$. For nail which is a hard tissue with comparably low water content and lack of the mineral component, $n$ $\cong 1.8$. For all other hard tissues with the lower content of water index of refraction is higher than for water due to inclusion of mineral tissue component, for tooth dentin $n \cong 2.4$, for bone $n \cong 2.5$, and for tooth enamel $n \cong 3.1$.

The scattering coefficient $\left(\mu_{s}\right)$ and scattering anisotropy factor $(g)$ of a tissue are dependent on refractive index mismatch - relative index of refraction $m$ [see Eqs. (3.25), (3.33), and (3.44)]. The index mismatch exists between cellular tissue components, such as cell membrane, cytoplasm, cell nucleus and other organelles, melanin granules, and the extracellular fluid. For fibrous (connective) tissue, index mismatch of interstitial medium and long strands of scleroprotein (collagen-, elastin-, or reticulin-forming fibers) is important. The scattering particles themselves (organelles, protein fibrils, membranes and protein globules) exhibit a higher density of proteins and lipids in comparison with the ground substance and, thus, a greater index of refraction $\left(n_{\mathrm{s}}=1.39-1.47\right)$. The refractive index of the interstitial liquid, as well as human blood plasma, is approximately 1.33-1.35, depending on the wavelength. The main scatterers in blood are red blood cells (RBCs). A hemoglobin ( $\mathrm{Hb})$ concentration of $32 \mathrm{~g} / \mathrm{dl}$ represents a typical $\mathrm{Hb}$ concentration within a human $\mathrm{RBC}$, and the refractive index of the solution is approximately 1.42 . For human whole blood, depending on the wavelength, the index is approximately $1.36-1.40$.

The optical immersion technique is based on the impregnation of a tissue by a biocompatible chemical agent, which may have hyperosmotic properties $[5,9$, 78-91, 93]. The OCAs frequently used are monosaccharides, such as glucose, dextrose, fructose; polysaccharides made of many glucose molecules dextrans; sugar alcohols (polyols) - glycerol, mannitol and sorbitol; alcohol - 1,3-butanediol; propylene glycol, polypropylene glycol, polyethylene glycol, 1,4butanediol and their combinations, such as combined lipophilic polypropylene glycol-based polymers and hydrophilic polyethylene glycol-based polymers; x-ray contrasting agents (verografin, trazograph, hypaque, omnipaque), etc. 
There are a several main mechanisms of light scattering reduction induced by an OCA: 1) dehydration of tissue constituents; 2) partial replacement of the interstitial fluid by the immersion substance; 3) structural modification (packing); and 4) dissociation of collagen. The first and the third mechanisms are characteristic for hyperosmotic agents. For fibrous tissue similar to sclera, dura mater, dermis, the second mechanism could be prevalent for many of tested chemical agents for which molecule size is much less than the mean cross-section of interfibrillar space. Both the first and the second processes mostly cause matching of the refractive indices of the tissue scatterers (cell constituents, collagen and elastin fibers) and the cytoplasm and/or interstitial fluid. The refractive index matching is manifested in the reduction of the scattering coefficient $\left(\mu_{s} \rightarrow 0\right)$ and increase of single scattering directness $(g \rightarrow 1)$ (see Fig. 3.12). For fibrous tissues such as skin dermis, eye sclera, dura mater, tendon, decrease of reduced scattering coefficient $\mu_{s}^{\prime}=(1-g) \mu_{s}$ can be significant.

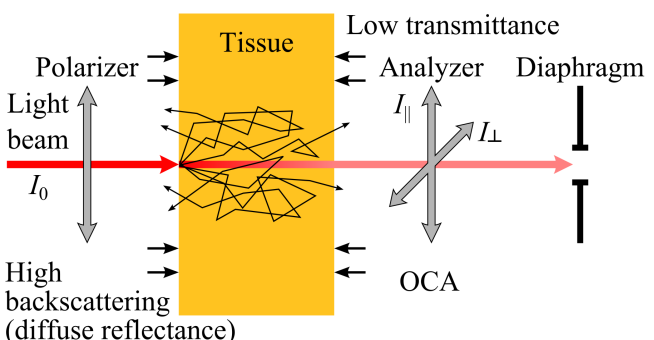

$\mathrm{a}$

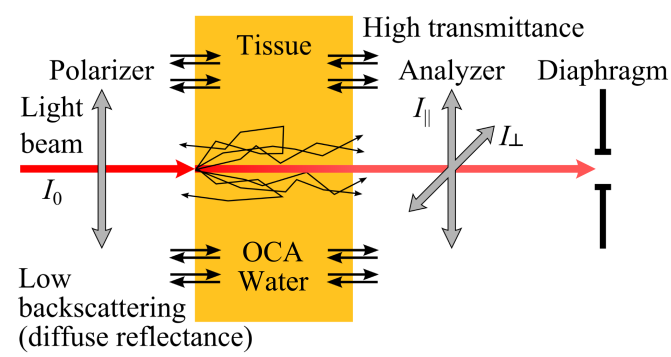

$\mathrm{b}$

Fig. 3.29 Schematic representation of diffusion of the immersion optical clearing agent (OCA) into and water out of tissue specimen with light increased transmittance (at collinear Polarizer and Analyzer) and decreased scattering due to optical immersion clearing; (a) is the initial specimen state before optical clearing, (b) is the final stage when it became transparent; input light beam is linear polarized by a Polarizer, the output beam is detected being processed by linear polarization Analyzer for two orthogonal polarization states - one in parallel with the polarization of the incident beam $I_{\|}$and another in perpendicular state $I_{\perp}$; Diaphragm in the far field of 1-1.5 $\mathrm{mm}$ in diameter serves for averaging of speckle structures for coherent light illumination or for detection of collimated light transmittance.
Structural modification is manifested as tissue shrinkage, it causes the near-order spatial correlation of scatterers (see Fig. 3.26) and, as a result, the increased constructive interference of the elementary scattered fields in the forward direction and destructive interference in the perpendicular direction of the incident light that may significantly increase tissue transmittance even at residual refractive index mismatch.

For some tissues and for the specific $\mathrm{pH}$ of applied OCA, tissue swelling may take place that could be considered as a competitive process in providing tissue optical clearing. The optical clearing process in collagen-based tissues may involve a change in the supramolecular structure. Collagen reversible solubility in sugars and sugar alcohols may take place. Agentinduced destabilization of collagen structures may lead to an additional reduction of optical scattering in tissue owing to less size of the main scatterers.

The osmotic pressure is a driving force in the generation of fluid flows and controlling intensities of these flows, providing a several mechanisms of optical clearing; however, rather strong osmotic pressure may destroy tissue structure. This is a major physicochemical mechanism of OCA toxicity.

For propagation of polarized light in fibrous tissue, it was shown that at a reduction of scattering, the degree of transmitted linearly polarized light significantly improves. Schematically such experiment is shown in Fig. 3.29. As far as the tissue is immersed, the number of scattering events decreases and the residual polarization degree of transmitted linearly polarized light increases. As a result, the kinetics of the average transmittance and degree of polarization of the tissue are correlated. Compare kinetics of clearing curves for linearly polarized component of transmitted intensity $I_{\|}$ and the total transmitted intensity $I_{T}$ in Fig. 3.30. OCAinduced optical clearing leads to an increase in the length of depolarization of a number of tissues. Figure 3.30 also demonstrates the reversibility of tissue clearing by successive OCA and physiological solution application.

As the spatially coherent laser beam was used in this experiment speckle pattern transformation accompanied optical clearing effects (see inserted far-field speckle patters in Fig. 3.30) [5]. Transmitted intensities $I_{T}, I_{\|}$, and $I_{\perp}$ were measured as mean speckle intensities averaged over the scanning trace $(1.5 \mathrm{~mm})$ in the paraxial region. It is well seen that the speckle patterns are transferred from small-size and more or less homogeneously distributed speckles, characteristic for multiple scattering, to big-size inhomogeneously distributed speckles with a big portion of ballistic photons in the central part of the pattern, which is due to low-scattering regime.

As other two examples of in vitro studies of optical clearing in the visible range, cerebral membrane (dura mater) and skin dermis could be presented. In the course of clearing during $20 \mathrm{~min}$, the reduced scattering 


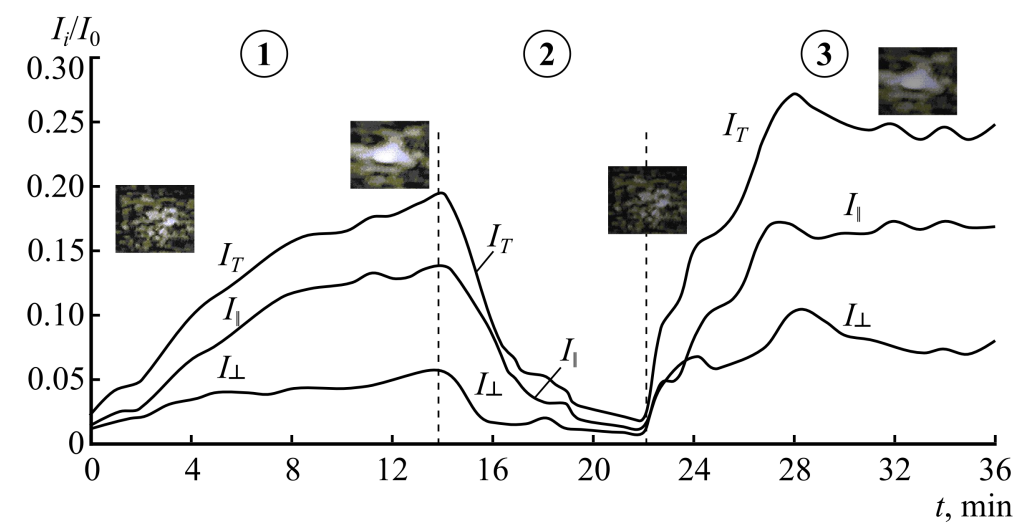

Fig. 3.30 The time-dependent transmittances $\left(I_{i} / I_{0}\right)$ of the human sclera specimen $(d=0.4 \mathrm{~mm})$ measured with a diaphragm at $\lambda=633 \mathrm{~nm}$ for linear polarization of the incident laser beam $I_{0} ; I_{i}=I_{\|}, I_{\perp}$ are two orthogonal polarization components of the transmitted light; $I_{\|}$in parallel to the polarization of the incident beam and $I_{\perp}-$ in perpendicular; $I_{T}$ $=I_{\|}+I_{\perp}$; the subsequent measurements for the specimen kept at first in 60\%-trazograph OCA solution, zone (1); in physiological solution $(0.9 \% \mathrm{NaCl})$, zone (2), and again in the OCA solution, zone (3); inserts show speckle patterns in the transmitted field before and after optical clearing without polarization filtration [5].

coefficient $\mu_{s}^{\prime}$ of dura mater decreases up to $40 \%$ (aqueous mannitol solution, $0.16 \mathrm{~g} / \mathrm{ml}, n=1.357$ ). Application of anhydrous glycerol $(n=1.47)$ during 20 min to skin dermis decreases $\mu_{s}^{\prime}$ of skin up to $75 \%$. Differences in the degree of tissue clearing can be explained by differences in refractive indices of the used OCAs, their osmolarity and initial state of tissue turbidity. The swelling of dura mater samples was observed, whereas skin shrinkage during the clearing.

Not only soft but also hard tissues could be effectively cleared, which opens the way for the development of the least-invasive techniques for laser diagnostics and therapy of brain and other tissues hidden under bone, cartilage or tendons. For example, optical immersion clearing of the cranial bone under action of anhydrous glycerol allows for decreasing $\mu^{\prime}$ of superficial tissue layers in an hour by approximately $25 \%$ for the wavelength range $1400-2000 \mathrm{~nm}$. In this case, the main role in the clearing process plays the diffusion of OCA into the interstitial space, owing to the bone-specific structure having a rather high porosity.

The multiple scattering is a detrimental factor that limits optical coherence tomography (OCT) imaging performances: imaging resolution, depth and localization. To improve the imaging capabilities, the multiple scattering of tissue and blood must be reduced. The immersion technique at application of biocompatible agents is a prospective technique for OCT, because very easily the depth of OCT images and their contrast can be essentially improved at immersion. The OCT measures reflectance, $R(z)$, from the tissue versus axial ranging distance, or depth, $z$. The relationship between $R(z)$ and $\mu_{t}$ can be approximately by [88]

$$
R(z)=I_{0} \alpha(z) \exp \left(-\mu_{t} z\right)
$$

where $I_{0}$ is the optical power launched into the tissue sample and $\alpha(z)$ is the reflectivity of the sample at the depth $z ; \alpha(z)$ is linked to the local refractive index and the backscattering property of the sample. If $\alpha(z)$ is a constant, $\mu_{t}$ can be obtained theoretically from the reflectance measurements at two different depths, $z_{1}$ and $z_{2}$ :

$$
\mu_{t}=\frac{1}{(\Delta z)} \ln \left[\frac{R\left(z_{1}\right)}{R\left(z_{2}\right)}\right],
$$

where $\Delta \mathrm{z}=\left|\mathrm{z}_{1}-\mathrm{z}_{2}\right|$.

Optical clearing (enhancement of transmittance) $\Delta T_{O C T}$ by an agent application can be estimated using the following expression

$$
\Delta T_{O C T}=\frac{R_{a}-R_{s}}{R_{s}} \times 100 \%
$$

where $R_{a}$ is the reflectance from the backward surface of the sample impregnated by an agent, and $R_{s}$ is that with a control sample.

Blood immerses or goes through practically all tissues. It is a highly scattering biological liquid with a strong anisotropy of scattering. Therefore, blood optical clearing is of great importance [5, 78-80, 88]. The refractive-index mismatch between erythrocyte cytoplasm and blood plasma, as well as specific size and structure, cause the scattering properties of blood. The refractive index of erythrocyte cytoplasm is defined mostly by hemoglobin concentration. The volume and 
shape of a single erythrocyte are defined by blood plasma osmolarity. Blood scattering also depends on aggregation or disaggregation capability of RBCs. Upon introduction of OCAs into blood, the refractive index of the blood plasma increases and becomes comparable with that of RBCs. For example, in whole blood diluted to twice of its volume by saline with the addition of $6.5 \%$ glycerol, the total attenuation coefficient $\mu_{t}=\mu_{a}+\mu_{s}$ was reduced from 42 to $20 \mathrm{~cm}^{-1}$, and the optical penetration at $820 \mathrm{~nm}$ was correspondingly increased to $117 \%$ in terms of $\Delta T_{O C T}$. For the other agents tested (glucose, dextrans, propylene glycol and trazograph), the enhancement of penetration $\Delta T_{O C T}=$ $20-150 \%$.

There is also the possibility of applying as an immersion agent, a small amount of hemoglobin, which could be released owing to local hemolysis of RBCs within the vessel area close to endoscopic optical probe. A $30-40 \%$ reduction of the scattering coefficient of blood in the spectral range from 400 to $1000 \mathrm{~nm}$, with an increase in the local RBC hemolysis up to $20 \%$ is expected. The optical clearing of blood is defined not only by refractive-index matching phenomenon, but also by changes in the size of RBCs and in their aggregation ability when chemicals are added.

It is known that at in vivo application of the designed optical immersion technology, additional factors such as metabolic reaction of living tissue on clearing agent application, the specificity of tissue functioning and its physiological temperature can significantly change kinetic characteristics and the magnitude of the clearing effect. In a living tissue, the relative refractive index is a function of tissue physiological or pathological state. Depending on the specificity of the tissue state, the refractive index of the scatterers and/or the background may be changed (increased or decreased), and therefore light scattering may correspondingly increase or decrease. For example, the $\mu_{s}^{\prime}$ calculated at a wavelength of $700 \mathrm{~nm}$ on the basis of in vivo spectral reflectance measurements for rabbit eye sclera (Fig. 3.31) [5] and in vitro spectral transmittance measurements for human sclera at administration of $40 \%$-glucose solution showed the clearing degree for in vivo study of 1.7 fold - that is, less than for in vitro ( 2.7 fold). Less efficiency for in vivo conditions may be explained by glucose washing out from the area of administration and physiological reaction of the living tissue.

In the terahertz range, where scattering is negligible and transport of radiation in tissues mostly depends on tissue absorption properties, in particular by tissue water absorption which absorption coefficient is rather high, $\mu_{a} \cong 100-300 \mathrm{~cm}^{-1}$. For normal muscle tissue absorption coefficient is also high, $\mu_{a} \cong 120-160 \mathrm{~cm}^{-1}$, however when tissue is dehydrated by application of a hyperosmotic agent absorption decreases up to $30-40 \%$ and tissue becomes more transparent [93].

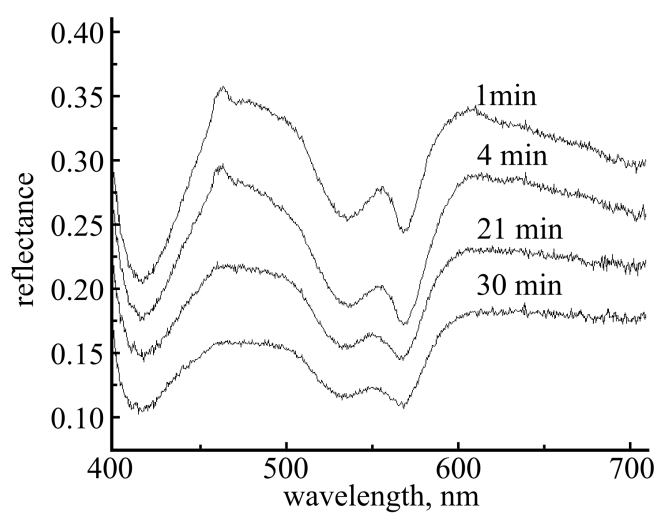

Fig. 3.31 Reflectance spectra for the rabbit eye sclera measured concurrently with administration of $40 \%$ glucose solution in $1,4,21$, and $30 \mathrm{~min}$ after a few drops of the solution into the eye [5].

Topical application of OCAs to skin is less efficient because of protective properties of the stratum corneum (SC), thus different chemical and physical enhancers of skin permeability are used [5, 9, 78-91]. The typical chemical enhancers are: ethanol, propylene glycol, dimethylsulfoxide (DMSO), linoleic and oleic acids, azone and thiazone. To reduce the barrier function of skin epidermis, physical methods such as tape stripping, microdermabrasion, low intensive and high intensive laser irradiation of skin surface, iontophoresis, ultrasound and photomechanical (shock) waves, needlefree injection, photothermal and mechanical microperforation, or laser fractional micro-damaging were proposed.

\section{Acknowledgments}

I am thankful to Valery Zakharov for his idea to write such a paper, to all my colleagues from SSU and IPMC RAS for fruitful collaboration, and to Alexander Kalyanov for help in preparation of illustrations. This work was supported by Russian Presidential grant NSh703.2014.2, the Government of the Russian Federation grant 14.Z50.31.0004, and the Ministry of Education and Science of the Russian Federation. 